Pontifícia Universidade Católlica $_{\text {dis }}$

DO RIO DE JANEIRO

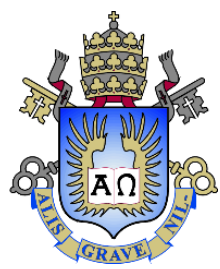

Maíra Fortes Bonafé

Solução Aproximada do Comportamento da Pressão em Testes de Injetividade com Vazão Variável

Dissertação de Mestrado

Dissertação apresentada ao Programa de Pós-graduação em Engenharia Mecânica da PUC-Rio como requisito parcial para obtenção do grau em Mestre em Engenharia Mecânica.

Orientador: Prof. Arthur Martins Barbosa Braga Co-orientador: Prof. Abelardo Borges Barreto Jr. 


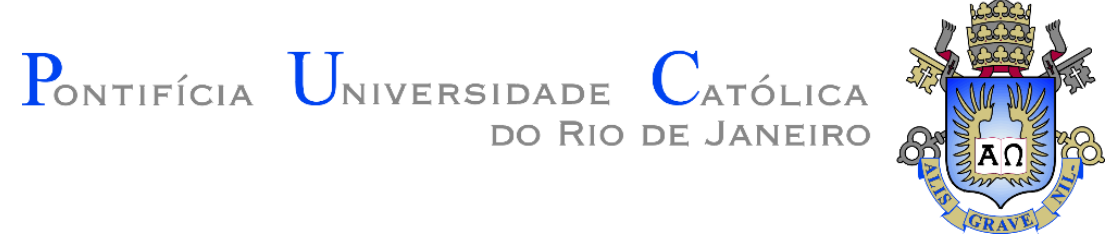

Maíra Fortes Bonafé

\title{
Solução Aproximada do Comportamento da Pressão em Testes de Injetividade com Vazão Variável
}

\begin{abstract}
Dissertação apresentada como requisito parcial para obtenção do título de Mestre pelo Programa de PósGraduação em Engenharia da PUC-Rio. Aprovada pela Comissão Examinadora abaixo.
\end{abstract}

Prof. Arthur Martins Barbosa Braga

Orientador

Departamento de Engenharia Mecânica - PUC-Rio

Prof. Abelardo Borges Barreto Jr. Co-Orientador Departamento de Engenharia Mecânica - PUC-Rio

Prof. Sinésio Pesco Departamento de Engenharia Mecânica - PUC-Rio

Prof. Márcio Arab Murad Laboratório Nacional de Computação Científica

Rio de Janeiro, 1 de abril de 2019 
Todos os direitos reservados. É proibida a reprodução total ou parcial do trabalho sem autorização da universidade, da autora e do orientador.

\section{Maíra Fortes Bonafé}

Graduou-se em Engenharia Elétrica na Universidade de Brasília (Unb) em 2011. Trabalhou na Tractebel Engineering de 2008 a 2014. Em 2014 ingressou na Agência Nacional do Petróleo, Gás Natural e Biocombustíveis (Anp) como Especialista em Regulação na Superintendência de Desenvolvimento e Produção (SDP). Em 2017 foi nomeada assessora da Coordenação de Campos Marítimos na SDP. Em 2018 foi nomeada assessora da Diretoria III da Anp.

Ficha Catalográfica

Bonafé, Maíra Fortes

Solução aproximada do comportamento da pressão em testes de injetividade com vazão variável / Maíra Fortes Bonafé ; orientador: Arthur Martins Barbosa Braga ; co-orientador: Abelardo Borges Barreto Jr. -2019.

98 f. : il. color. ; $30 \mathrm{~cm}$

Dissertação (mestrado)-Pontifícia Universidade Católica do Rio de Janeiro, Departamento de Engenharia Mecânica, 2019.

Inclui bibliografia

1. Engenharia Mecânica - Teses. 2. Testes de injetividade. 3. teste de formação. I. Braga, Arthur Martins Barbosa. II. Barreto Júnior, Abelardo Borges. III. Pontifícia Universidade Católica do Rio de Janeiro. Departamento de Engenharia Mecânica. IV. Título. 
Para meus pais, Leonardo e Mara, meu irmão Fábio, e meu esposo, Renato, pelo apoio e confiança. 


\section{Agradecimentos}

Ao meu orientador Professor Arthur Martins Barbosa Braga pelo estímulo e parceria para a realização deste trabalho.

Ao meu co-orientador Professor Abelardo Borges Barreto Jr., pelas importantes contribuições e palavras de apoio.

Ao CNPq e à PUC-Rio, pelos auxílios concedidos, sem os quais este trabalho não poderia ter sido realizado.

À Anp, pelo incentivo e suporte essenciais para conclusão desse trabalho.

Aos professores que participaram da Comissão examinadora.

A todos os professores e funcionários do Departamento pelos ensinamentos e pela ajuda.

A todos os amigos e familiares que de uma forma ou de outra me estimularam ou me ajudaram 


\section{Resumo}

Bonafé, Maíra Fortes; Solução Aproximada do Comportamento da Pressão em Testes de Injetividade com Vazão Variável. Rio de Janeiro, 2019. 98p. Dissertação de Mestrado - Departamento de Engenharia Mecânica, Pontifícia Universidade Católica do Rio de Janeiro.

Os testes de formação são uma operação realizada com o objetivo de identificar os fluidos, determinar os parâmetros de reservatório associados à produtividade e avaliar a extensão da jazida. Para executar o teste, o poço é completado temporariamente para permitir a produção dos fluidos de forma segura, e o intervalo a ser testado é isolado e um diferencial de pressão entre a formação e o interior do poço é estabelecido, forçando os fluidos da formação a serem produzidos.

A busca por uma forma de substituir os testes de produção tradicionais, evitando a queima de gás natural e o aumento da segurança operacional, somada ao fato de completações de poços injetores de água na zona produtora de óleo ser uma prática comum do desenvolvimento de campos marítimos, levaram os testes de injetividade a ter um importante papel no gerenciamento dos reservatórios desses campos. Embora os métodos de análise de fluxo monofásico com pequena compressibilidade sejam bem documentados na literatura, soluções bifásicas e métodos de análise ainda precisam ser desenvolvidos. Nessa dissertação, é proposta a modelagem do problema de testes de injetividade com vazão variável, utilizando soluções analíticas aproximadas.

\section{Palavras-chave}

Testes de injetividade; teste de formação; 


\section{Abstract}

Bonafé, Maíra Fortes; Variable Rate Approximate Solution for Pressure Response in a Water Injection Well. Rio de Janeiro, 2019. 98p. MSc. Dissertation - Departamento de Engenharia Mecânica, Pontifícia Universidade Católica do Rio de Janeiro.

The pressure response during a well test provides information on well productivity and reservoir performance. To replace traditional production tests, avoiding the gas flaring and increasing operational safety, the injectivity test began to play an important role in the management of the reservoirs in these fields. Although analytical models are able to describe injection and falloff periods, variable rate models still need to be developed. This work attempts to present a variable rate approximate solution for pressure response in a water injection well. The accuracy of the proposed solution was assessed by comparison with a numerical simulator. The suggested model was also used to determine the reservoir equivalent permeability.

\section{Keywords}

Water injection well; Formation testing. 


\section{Sumário}

1 Introdução 15

2 Escoamento Monofásico 18

2.1. Hipóteses do Modelo 18

2.2. Vazão Constante 19

2.3. Vazão Variável 26

3 Escoamento Bifásico: Períodos de Injeção e Falloff 30

3.1. Hipóteses do Modelo 31

3.2. Período de Injeção 32

3.2.1. Período de Injeção com Deslocamento Pistão 42

3.3. Período de Falloff 44

4 Escoamento Bifásico: Vazão Variável $\quad 51$

5 Validação dos Modelos 54

5.1. Comparação entre Modelos Analíticos e Modelo Numérico 54

5.2. Comparação entre Modelos de Vazão Variável para Escoamento Bifásico e para Escoamento Monofásico. 58

5.3. Caso 1 - Modelo com três vazões variáveis 61

5.4. Caso 2 - Modelo com quatro vazões variáveis 63

5.5. Caso 3 - Modelo com seis vazões variáveis 64

5.6. Cálculo da Permeabilidade Efetiva 69

6 Conclusão $\quad 75$

$\begin{array}{ll}\text { Referências Bibliográficas } & 76\end{array}$ 
Apêndice A

Apêndice B

84

Apêndice C

87

Apêndice D

89

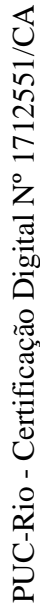




\section{Lista de Figuras}

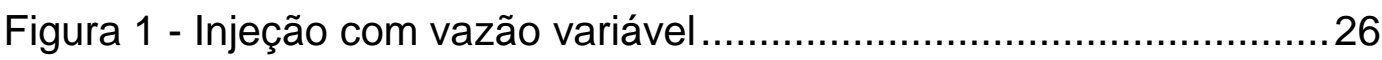

Figura 2- Injeção de água em um reservatório de óleo ............................30

Figura 3 - Variação do comportamento da pressão.................................31

Figura 4 - Gráfico de Permeabilidades Relativas .....................................34

Figura 5 - Representação da injeção de água no reservatório ...................37

Figura 6 - Variação da posição da frente de avanço de água ...................40

Figura 7 - Deslocamento em pistão.................................................... 42

Figura 8 - Representação do período de falloff ....................................... 44

Figura 9 - Representação da solução no período de estática ....................47

Figura 10 - Comportamento da pressão no modelo onde $\mathrm{M}>1 \ldots \ldots \ldots \ldots . . .55$

Figura 11 - Comportamento da pressão no modelo onde $M<1 \ldots \ldots \ldots \ldots . . .55$

Figura 12 - Comparação no período de injeção para $M>1 \ldots \ldots \ldots \ldots \ldots \ldots \ldots . . .56$

Figura 13 - Comparação no período de falloff para $M>1$.......................56

Figura 14 - Comparação no período de injeção para $\mathrm{M}<1$ …................57

Figura 15 - Comparação no período de falloff para $M<1 \ldots \ldots \ldots \ldots \ldots \ldots \ldots . . .57$

Figura 16 - Período de fluxo monofásico X Período de injeção $-M>1$..58

Figura 17 - Período de fluxo monofásico $X$ Período de injeção $-M<1$..59

Figura 18 - Período de fluxo monofásico X Período de falloff ...................60

Figura 19 - Período de fluxo monofásico X Período de falloff ...................61

Figura 20 - Esquema de vazões do Caso 1. .........................................62

Figura 21 - Caso 1 - Período com três vazões ........................................63

Figura 22 - Esquema de vazões do Caso 2. .........................................63

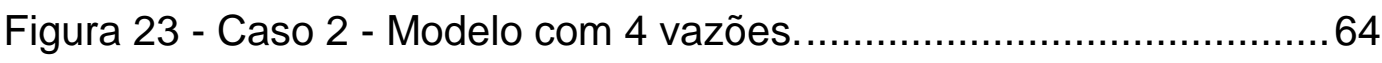

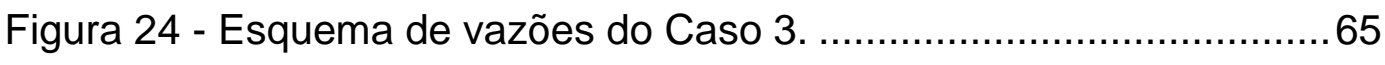

Figura 25 - Caso 3 - Modelo com seis vazões.......................................65

Figura 26 - Comparação entre derivadas - Primeiro período .....................66

Figura 27 - Comparação entre derivadas - Segundo período ....................67

Figura 28 - Comparação entre derivadas - Terceiro período ....................67 
Figura 29 - Comparação entre derivadas - Quarto período.......................68

Figura 30 - Comparação entre derivadas - Quinto período ........................68

Figura 31 - Comparação entre derivadas - Sexto período ……………....69

Figura 32 - Variação da pressão de fundo de poço - 1ํo período ...............70

Figura 33 - Variação da pressão de fundo de poço - $2^{\circ}$ período ...............71

Figura 34 - Variação da pressão de fundo de poço - 3o período ................72

Figura 35 - Variação da pressão de fundo de poço - $4^{\circ}$ período ................72

Figura 36 - Variação da pressão de fundo de poço - $5^{0}$ período ................73

Figura 37 - Variação da pressão de fundo de poço - 60 período ................73

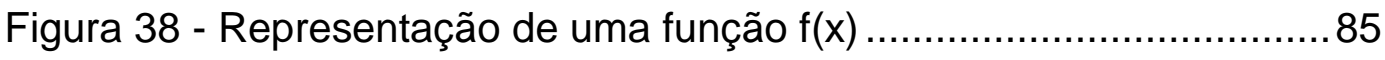

Figura 39 - Representação dos pontos de medição de pressão ..............86 


\section{Lista de Tabelas}

Tabela 1 - Constantes para Adequação de Unidades aos Sistemas de Medidas (adaptado de ROSA, 2011) ......................................25

Tabela 2- Cálculo das permeabilidades por período. .......................... 74 


\section{Sumário das Notações}

B, Fator volume de formação

$c_{o}$, Compressibilidade do óleo

$c_{r}, \quad$ Compressibilidade da rocha

$c_{t o}, \quad$ Compressibilidade total

$c_{w}, \quad$ Compressibilidade da água

$f_{w}$, Fluxo fracionário de água

$h$, Altura do intervalo canhoneado (m)

$k$, Coeficiente de permeabilidade (mD)

$k_{l}, \quad$ Permeabilidade efetiva da fase $(l=o, w)$

$k_{r l}$, Permeabilidade relativa da fase $(l=o, w)$

M , Razão de mobilidade

$p_{c}, \quad$ Pressão capilar

$p_{i}, \quad$ Pressão inicial de equilíbrio do reservatório $\left(\mathrm{kgf} / \mathrm{cm}^{2}\right)$

$p_{l}, \quad$ Pressão na fase $(l=o, w)$

$\rho_{l}, \quad$ Massa específica $(l=o, w)$

$p_{w f}$, Pressão de fluxo no fundo do poço $\left(\mathrm{kgf} / \mathrm{cm}^{2}\right)$

$p_{w s}$, Pressão no fundo do poço durante a estática $\left(\mathrm{kgf} / \mathrm{cm}^{2}\right)$

$q_{\text {inj }}$, Vazão de injeção ( $\left.\mathrm{m}^{3} / \mathrm{dia}\right)$

$q_{o}$, Vazão de óleo

$q_{t}$, Vazão total

$q_{w}$, Vazão de água

$r_{e}, \quad$ Raio externo

$r_{r}, \quad$ Posição da frente de avanço $(\mathrm{m})$

$r_{r t p}$, Posição da frente de avanço no momento do fechamento do poço

(m)

$r_{w}, \quad$ Raio do poço $(\mathrm{m})$

$S_{l}, \quad$ Saturação da fase $(l=o, w)$ nas condições de reservatório

$S_{\text {or }}$ Saturação residual de óleo nas condições de reservatório 
$S_{w i}$, Saturação inicial de água nas condições de reservatório

$t_{e q}$, Tempo equivalente

$t_{\text {horner }}$ Tempo de Horner

$t_{i}, \quad$ Tempo inicial (h)

$t_{p}, \quad$ Tempo de produção (h)

$v_{l}$, Velocidade macroscópica (superficial) da fase $(l=o, w)$

$\alpha$ ou $\alpha_{p}$, Constante para Adequação de Unidades aos Sistemas de Medidas; SI ou Darcy , $\quad 2 \pi \rightarrow$ Petrobras $=19,03$

$\beta$ ou $\alpha_{t}$, Constante para Adequação de Unidades aos Sistemas de Medidas; SI ou Darcy , $\quad 1 \rightarrow$ Petrobras $=0,0003484$

$\gamma, \quad 0.57722=$ Constante de Euler

$\eta, \quad$ Coeficiente de difusidade hidráulica $=K /\left(\phi^{*} \mu_{o}{ }^{*} c_{t o}\right)$

$\rho_{l}, \quad$ Densidade da fase $(l=o, w)$

$\mu_{l}, \quad$ Viscosidade da fase $(l=o, w)(\mathrm{cP})$

$\lambda_{l}, \quad$ Mobilidade da fase $(l=o, w)$

$\lambda_{w}$, Mobilidade total

$\phi$, Porosidade

$E_{i}$, Função Integral Exponencial 


\section{Introdução}

O Brasil ratificou o Acordo de Paris para redução das emissões de gases de efeito estufa, com vistas à manutenção do aumento da temperatura global em menos de $2^{\circ} \mathrm{C}$, envidando esforços para limitar esse incremento a $1,5^{\circ} \mathrm{C}$ acima dos níveis pré-industriais. Além disso, as Melhores Práticas da Indústria do Petróleo pressupõem a conservação de recursos petrolíferos e gaseíferos, o que implica a utilização de métodos e processos adequados à minimização das perdas na superfície.

Esses fatos, somados a constante procura por aumento na Segurança Operacional, levaram a busca por uma forma de substituir os testes de produção tradicionais, como por exemplo testes com re-injeção, closer chamber e testes de injetividade.

Os testes de injetividade, objeto desse trabalho se destacam pelo fato de completações de poços injetores de água na zona produtora de óleo serem uma prática comum no desenvolvimento de campos marítimos.

Entretanto, métodos de análise de testes de injetividade com vazão variável são pouco documentados, e soluções analíticas para o estudo do comportamento da pressão de fundo de poço durante esses testes ainda precisam ser desenvolvidas, o que motivou esse trabalho.

Em um teste de injetividade, uma fase única (água) é injetada continuamente por um determinado período de tempo em um reservatório saturado de óleo e em seguida a injeção é interrompida, ocorrendo um período falloff, que leva a uma queda de pressão no reservatório. Parâmetros do reservatório, tais como permeabilidade e efeito de película, são obtidos a partir do estudo do comportamento das pressões de fundo de poço, medidas durante testes de injetividade. Além disso, é possível avaliar a conectividade do reservatório e o potencial de injeção do poço 
A seguir apresenta-se um resumo dos artigos que abordaram o comportamento da pressão durante testes de injetividade e guiaram esse trabalho.

Thompson e Reynolds (1997) analisaram o comportamento da pressão para fluxos monofásicos e multifásicos radiais em reservatórios heterogêneos, incluindo a análise durantes testes de injetividade, desenvolvendo uma teoria geral para esse comportamento.

Peres e Reynolds (2003) utilizaram esses conceitos para construir soluções analíticas para o comportamento da pressão durante o período de falloff, após um período de injeção de água, comparando essas soluções com a resposta gerada por um simulador.

Soluções analíticas aproximadas para poços verticais com completação restrita foram desenvolvidas por Peres e Boughrara (2004). Para isso, os autores utilizaram a solução de fluxo monofásico acrescida de um termo multifásico que representaria a zona bifásica e o movimento da frente de água, onde o modelo para o movimento da frente de água foi baseado na teoria de Buckley-Leverett (1942). Por meio de comparações com soluções numéricas geradas por um modelo de simulação, os autores constataram que as soluções analíticas aproximadas forneciam resultados satisfatórios.

Posteriormente, Peres e Reynolds (2006) utilizaram o princípio da superposição para detalhar a solução no período de falloff. Embora o problema da injeção seja não linear, esses autores se basearam nos trabalhos de Abbaszadeh e Kamal (1989) e Bratvold e Horne (1990), que mostraram que a solução analítica no período de falloff poderia ser construída com acurácia suficiente se for considerado que o perfil de mobilidade no instante de fechamento do poço injetor não se altera.

Uma solução analítica para poços verticais injetores de água completados na zona de óleo foi proposta por Barreto e Peres (2011), onde o deslocamento das duas fases imiscíveis foi modelado por um sistema de equações diferenciais parciais. Considerando que esse sistema é fracamente não linear, ele foi decomposto em duas regiões, a monofásica e a bifásica. 
Esse trabalho utiliza as soluções analíticas aproximadas propostas por estudos anteriores para a modelagem do problema de testes de injetividade com vazão variável, utilizando soluções numéricas geradas por um modelo de simulação para sua validação. 


\section{Escoamento Monofásico}

Esse capítulo apresenta o desenvolvimento da formulação básica para descrever o comportamento da pressão no fundo do poço em um modelo de reservatório com escoamento monofásico, a partir da Equação da Continuidade e da Lei de Darcy. Primeiramente é apresentado o desenvolvimento da solução considerando a vazão de produção ou injeção como constante. No item seguinte, é desenvolvida a solução para o caso monofásico com vazão variável.

\section{1.}

\section{Hipóteses do Modelo}

O modelo de reservatório utilizado nessa etapa do trabalho, apresenta as características detalhadas abaixo e, além disso, nesse estudo, o efeito gravitacional e a pressão capilar $\left(p_{c}\right)$ são desprezados.

- Reservatório poroso homogêneo e isotrópico;

- Espessura constante;

- Viscosidade constante;

- Permeabilidade constante;

- Escoamento isotérmico;

- Pequenos gradientes de pressão

- Poço radial;

- Fluido flui perpendicularmente à área da seção do poço;

- Grãos e fluidos pouco compressíveis;

- Porosidade não se altera;

- Fluidos e rochas não reagentes entre si; 
- Reservatório infinito;

- Sistema inicialmente em equilíbrio, ou seja, $p(r, t=0)=p_{i}$.

\section{2.}

\section{Vazão Constante}

Nesse item, é desenvolvida a formulação básica para descrever o comportamento da pressão no fundo do poço em um modelo de reservatório com escoamento monofásico com vazão constante.

Durante o período de produção ou injeção, há movimentação de fluido dentro do meio poroso, a Equação da Continuidade descreve a variação de massa no meio poroso devido a esse fluxo como sendo igual a diferença entre a massa que entra e a massa que sai. Sendo que para o fluxo monofásico em meios porosos com geometria radial, pode-se escrever:

$$
\frac{1}{r} \frac{\partial}{\partial r}\left(r \rho_{l} v_{l}\right)=-\frac{\partial}{\partial t}\left(\phi \rho_{l}\right)
$$

A equação ( 1 ) possui duas variáveis dependentes, velocidade aparente e massa específica do fluido, visando colocar essa equação em função apenas da pressão, utiliza-se a Lei de Darcy, que rege o transporte do fluido no meio poroso. De acordo essa lei, nos casos em que o efeito gravitacional é desprezível, a velocidade aparente do fluido no reservatório é dada por:

$$
v_{l}(r)=\frac{-k_{l}}{\mu_{l}} \frac{\partial p}{\partial r}
$$

Substituindo a equação ( 2 ) em ( 1 ): 
$\frac{1}{r} \frac{\partial}{\partial r}\left(\rho_{l} \frac{k_{l}}{\mu_{l}} r \frac{\partial p}{\partial r}\right)=\frac{\partial}{\partial t}\left(\phi \rho_{l}\right)$

No caso em estudo, onde considera-se um reservatório homogêneo e isotrópico, com compressibilidade e viscosidade do fluido constantes.

Dessa forma, pode-se escrever a equação ( 3 ) como:

$$
\frac{k_{l}}{\mu_{l}} \frac{1}{r} \frac{\partial}{\partial r}\left(\rho_{l} r \frac{\partial p}{\partial r}\right)=\frac{\partial}{\partial t}\left(\phi \rho_{l}\right)
$$

Utilizando a derivada do produto, pode-se expandir o lado direito da equação ( 3 ):

$$
\frac{\partial}{\partial t}\left(\phi \rho_{l}\right)=\left[\phi \frac{\partial \rho_{l}}{\partial t}+\rho_{l} \frac{\partial \phi}{\partial t}\right]
$$

De acordo com Rosa (2011) as equações da compressibilidade isotérmica de um fluido e da rocha são dadas pelas equações ( 6 ) e ( 7 ), respectivamente.

$$
c_{l}=\frac{1}{\rho_{l}} \frac{\partial \rho_{l}}{\partial p}
$$

$$
c_{r}=\frac{1}{\phi} \frac{\partial \phi}{\partial p}
$$

A partir dessas equações, podem ser obtidas as seguintes expressões:

$$
\begin{aligned}
& \frac{\partial p}{\partial t}=\frac{1}{c_{l} \rho_{l}} \frac{\partial \rho_{l}}{\partial t} \\
& \frac{\partial \phi}{\partial t}=c_{r} \phi \frac{\partial p}{\partial t}
\end{aligned}
$$

De forma que a equação ( 5 ) pode ser escrita como: 


$$
\begin{aligned}
& \frac{\partial}{\partial t}\left(\phi \rho_{l}\right)=\left[\phi c_{l} \rho_{l} \frac{\partial p}{\partial t}+\rho_{l} c_{r} \phi \frac{\partial p}{\partial t}\right] \\
& \frac{\partial}{\partial t}\left(\phi \rho_{l}\right)=\phi \rho_{l}\left(c_{l}+c_{r}\right) \frac{\partial p}{\partial t}
\end{aligned}
$$

A soma da compressibilidade do fluido com a compressibilidade da rocha é conhecida como compressibilidade total, $c_{t}$, de forma que:

$$
\frac{\partial}{\partial t}\left(\phi \rho_{l}\right)=\phi \rho_{l} c_{t} \frac{\partial p}{\partial t}
$$

Expandindo o lado esquerdo da equação ( 4 ) e utilizando novamente as definições de compressibilidade, pode-se escrever a equação ( 4 ) como:

$$
\frac{1}{r} \frac{\partial}{\partial r}\left(r \frac{\partial p}{\partial r}\right)+c_{l}\left(\frac{\partial p}{\partial r}\right)^{2}=\frac{\phi \mu_{l} c_{t}}{k_{l}} \frac{\partial p}{\partial t}
$$

Esse sistema é não linear devido à dependência dos coeficientes da equação em relação à pressão.

O termo quadrático também contribui com a não linearidade. Entretanto, quando as compressibilidades forem pequenas e o gradiente de pressão não for muito grande, o que usualmente é utilizado com líquidos, esse termo pode ser desprezado, escrevendo-se:

$$
\frac{1}{r} \frac{\partial}{\partial r}\left(r \frac{\partial p}{\partial r}\right)=\frac{\phi \mu_{l} c_{t}}{k_{l}} \frac{\partial p}{\partial t}
$$

Essa equação é conhecida como Equação da Difusidade para fluido com pequena compressibilidade e admite inúmeras soluções.

A mobilidade da fase é definida por: 


$$
\lambda_{l}=\frac{k_{l}}{\mu_{l}}
$$

No caso do escoamento monofásico de óleo, podemos ainda escrever $c_{t}=\hat{c}_{t o}$, e a mobilidade como:

$$
\hat{\lambda}_{o}=\frac{k_{o}}{\mu_{o}}
$$

Para obter a solução para um caso particular é necessário especificar as condições de contorno.

A condição de contorno externa (C.C.E), reflete a condição de fluxo no limite extremo do reservatório. Na hipótese adotada neste trabalho considera-se um reservatório infinito. Esta C.C.E. significa apenas que durante o tempo do teste as fronteiras externas não afetam o comportamento do poço em teste.

A condição de contorno interna (C.C.I), diz respeito ao modo como o poço está sendo produzido durante o teste, no caso em tela adotou-se a vazão constante.

A condição inicial (C.I) indica a distribuição da pressão ao longo do reservatório no tempo zero, que no caso é a pressão inicial de equilíbrio do reservatório.

Sendo assim, o sistema é composto pelas seguintes equações:

$$
\begin{array}{lc}
\text { EDP } & \frac{1}{r} \frac{\partial}{\partial r}\left(r \frac{\partial p}{\partial r}\right)=\frac{\phi}{\hat{c}_{t o}} \frac{\partial p}{\hat{\lambda}_{o}} \frac{\partial}{\partial t} \\
\text { CI } & p(r, t=0)=p_{i} \\
\text { CCE } & \lim _{\infty} p(r, t)=p_{i} \\
\text { CCI } & -2 \pi k \hat{\lambda}_{o} h \lim _{r \rightarrow 0}\left(r \frac{\partial p}{\partial r}\right)=\tilde{q}
\end{array}
$$

A solução desse sistema de equações é:

$$
\frac{2 \pi k \hat{\lambda}_{o} h\left[p(r, t)-p_{i}\right]}{\tilde{q}}=-\frac{1}{2} E_{i}\left(-\frac{r^{2} \phi \hat{c}_{t o}}{4 k \hat{\lambda}_{o} t}\right)
$$


A solução do problema também poderia ser desenvolvida partindo-se diretamente da equação ( 2 ), sendo a vazão de óleo igual a vazão total:

$$
q_{o}(r, t)=A \cdot v_{o}
$$

Onde A é a área da seção transversal do poço $\left(2 \pi h r^{\prime}\right)$, e $v_{o}$ é a velocidade aparente do fluido dada pela equação ( 2 ), podendo-se escrever:

$$
q_{o}(r, t)=-\frac{2 \pi h r k_{o}}{\mu_{o}} \frac{\partial p}{\partial r}
$$

Rearranjando os dois lados da equação,

$$
\frac{\partial p}{\partial r}=-\frac{1}{r} \frac{q_{o}(r, t) \mu_{o}}{2 \pi k_{o} h}
$$

Pode-se ainda integrar a equação do raio do poço $\left(r_{w}\right)$ até uma distância r qualquer, no reservatório:

$$
\int_{r_{w}}^{r} \frac{\partial p}{\partial r} d r=-\int_{r_{w}}^{r} \frac{1}{r} \frac{q_{o}(r, t) \mu_{o}}{2 \pi k_{o} h} d r
$$

Como vimos, no caso do escoamento monofásico, $k_{o}$ é constante, de forma que se pode escrever:

$$
p(r, t)-p\left(r_{w}, t\right)=-\frac{\mu_{o}}{2 \pi k_{o} h} \int_{r_{w}}^{r} \frac{q_{o}(r, t)}{r} d r
$$

Considerando a CCE de reservatório infinito,

$$
p(r, t)=p(r \rightarrow \infty, t)=p_{i}
$$


Pode-se reescrever a equação ( 26 ),

$$
p\left(r_{w}, t\right)-p_{i}=\frac{\mu_{o}}{2 \pi k_{o} h} \int_{r_{w}}^{\infty} \frac{q_{o}(r, t)}{r} d r
$$

Pode-se utilizar as equações ( 21 ) e ( 23 ) para encontrar $q_{o}(r, t)$.

Derivando a equação ( 21 ) em relação ao raio, usando a regra de Liebnitz,

$$
\frac{\partial p(r, t)}{\partial r}=\frac{\tilde{q}}{2 \pi k \hat{\lambda}_{o} h} \frac{\partial}{\partial r}\left[-\frac{1}{2} E_{i}\left(-\frac{r^{2} \phi \hat{c}_{t o}}{4 k \hat{\lambda}_{o} t}\right)\right]
$$

Onde $E_{i}$ é a função integral exponencial dada por $E_{i}(u)=\int_{u}^{\infty} \frac{e^{-u}}{u} d u, \log o:$

$$
\begin{aligned}
& \frac{\partial p(r, t)}{\partial r}=\frac{\tilde{q}}{2 \pi k \hat{\lambda}_{o} h} \frac{1}{2}\left[\frac{\partial}{\partial r} \int_{-\frac{r^{2} \phi \hat{c}_{t o}}{4 k \hat{\lambda}_{o} t}}^{\infty} \frac{e^{-u}}{u} d u\right] \\
& \frac{\partial p(r, t)}{\partial r}=-\frac{\tilde{q}}{2 \pi k \hat{\lambda}_{o} h} \frac{1}{r} e^{\left(-\frac{r^{2} \phi \hat{c}_{t o}}{4 k \hat{\lambda}_{o} t}\right)}
\end{aligned}
$$

Substituindo a equação ( 31 ) em ( 24 ):

$$
q_{o}\left(r^{\prime}, t\right)=-\frac{2 \pi h r^{\prime} k_{o}}{\mu_{o}} \frac{\tilde{q}}{2 \pi k \hat{\lambda}_{o} h} \frac{1}{r} e^{\left(-\frac{r^{2} \phi \hat{c}_{o}}{4 k \hat{\lambda}_{o} t}\right)}
$$

$q_{o}\left(r^{\prime}, t\right)=\tilde{q} e^{\left(-\frac{r^{2} \phi \hat{c}_{t o}}{4 k \hat{\lambda}_{o} t}\right)}$

Substituindo a equação ( 33 ) em ( 28 ):

$$
p\left(r_{w}, t\right)-p_{i}=\frac{\tilde{q}}{2 \pi k \hat{\lambda}_{o} h} \int_{r_{w}}^{\infty} e^{\left(-\frac{r^{2} \phi \hat{c}_{t o}}{4 k \hat{\lambda}_{o} t}\right)} \frac{d r}{r}
$$




$$
p\left(r_{w}, t\right)-p_{i}=\frac{\tilde{q}}{2 \pi k \hat{\lambda}_{o} h}\left[-\frac{1}{2} E_{i}\left(-\frac{r^{2} \phi \hat{c}_{t o}}{4 k \hat{\lambda}_{o} t}\right)\right]
$$

Tabela 1 - Constantes para Adequação de Unidades aos Sistemas de Medidas (adaptado de ROSA, 2011)

\begin{tabular}{|c|c|c|r|}
\hline \multirow{2}{*}{ Variável } & \multicolumn{3}{|c|}{ Sistema de Unidades } \\
\cline { 2 - 4 } & SI ou Darcy & Brasileiro & Americano \\
\hline$\alpha_{t} /$ beta & 1 & 0,0003484 & 0,00026374 \\
\hline$\alpha_{p} /$ alfa & $2 \pi$ & 19,03 & 141,2 \\
\hline
\end{tabular}

Utilizando a constante $\alpha_{p}$ para adequação de unidades ao sistema de medidas Brasileiro, conforme indicado na Tabela 1, escreve-se:

$$
p\left(r_{w}, t\right)-p_{i}=\frac{\alpha_{p} \tilde{q}}{h k \hat{\lambda}_{o}}\left[-\frac{1}{2} E_{i}\left(-\frac{r^{2} \phi \hat{c}_{t o}}{4 \alpha_{t} k \hat{\lambda}_{o} t}\right)\right]
$$

Nesse caso, onde a vazão é constante, $\tilde{q}=q_{w} B_{w}$, logo, encontra-se a mesma solução dada pela equação ( 21 )

$$
p\left(r_{w}, t\right)-p_{i}=\frac{\alpha_{p} q_{w} B_{w}}{h k \hat{\lambda}_{o}}\left[-\frac{1}{2} E_{i}\left(-\frac{r^{2} \phi \hat{c}_{t o}}{4 \alpha_{t} k \hat{\lambda}_{o} t}\right)\right]
$$

Para pressões medidas no poço, para tempos maiores que um segundo, a função $E_{i}$ pode ser feita a seguinte aproximação:

$$
E_{i}(-x) \cong \ln (x)+\gamma
$$

Logo,

$$
p_{w f}-p_{i}=-\frac{\alpha_{p} q_{w} B_{w}}{h k \hat{\lambda}_{o}}\left[\frac{1}{2} \ln \left(\frac{4 \alpha_{t} k \hat{\lambda}_{o} t}{e^{\gamma} \hat{c}_{t o} \phi r_{w}^{2}}\right)\right]
$$


Que representa a solução monofásica calculada com propriedades da fase óleo @ $S_{w i}$ :

$$
\Delta \widehat{p_{o}}(\mathrm{t})=-\frac{\alpha_{p} q_{w} B_{w}}{h k \hat{\lambda}_{o}}\left[\frac{1}{2} \ln \left(\frac{4 \alpha_{t} k \hat{\lambda}_{o} t}{e^{\gamma} \widehat{c_{t o}} \phi r_{w}^{2}}\right)\right]
$$

\section{3.}

\section{Vazão Variável}

Como o objetivo do trabalho é a modelagem de testes de injetividade com vazão variável, nesse item é apresentada a formulação para representar o comportamento da pressão de fundo de poço quando a vazão de produção de fluidos no reservatório é variável. Para isso, considera-se um poço injetor cuja vazão, $q_{i n j}(r, t)$, varia com o tempo, conforme exemplificado na Figura 1.

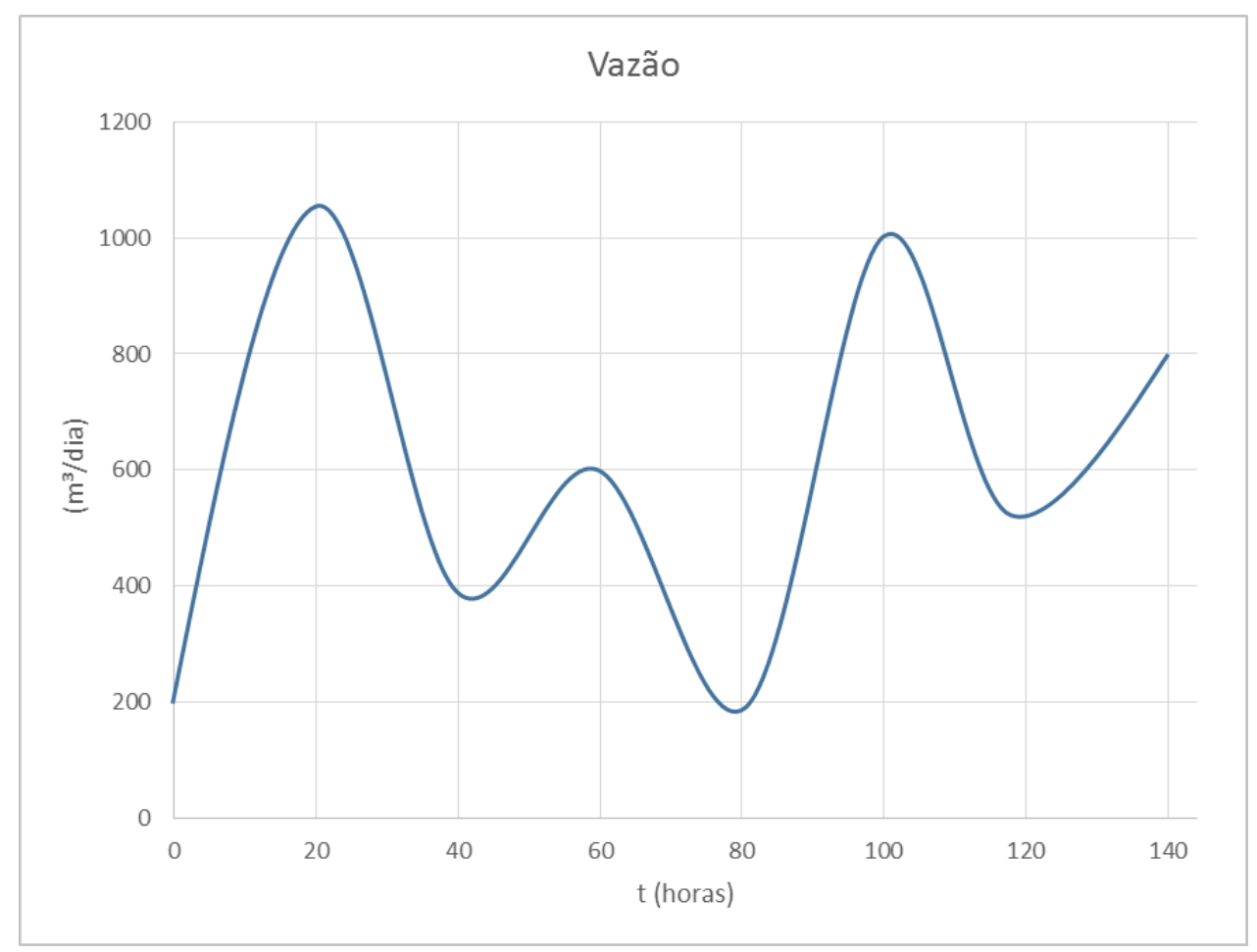

Figura 1 - Injeção com vazão variável

A solução para vazão variável é dada pelo Princípio de Duhamel, que é uma expressão matemática que relaciona essa solução com a solução 
para vazão constante. Em termos adimensionais (Apêndice C), essa expressão é fornecida pela equação ( 41).

$$
p_{D}\left(r_{D}, t_{D}\right)=\int_{0}^{t_{D}} q_{D}(\tau) \frac{\partial p_{D C}}{\partial t_{D}}\left(r_{D}, t_{D}-\tau\right) d \tau
$$

Entretanto, nesse trabalho a solução será apresentada na forma discreta e não contínua.

A solução apresentada na equação ( 40 ) foi deduzida para o caso de um reservatório de extensão infinita sendo produzido por um único poço com vazão constante.

Essa solução pode ser utilizada para construir soluções para outras situações como a produção com vazão variável.

Nesse caso, a vazão em cada instante pode ser escrita pela superposição dos efeitos das diversas variações de vazão $\left(q_{1}-q_{0}\right)$, ( $q_{2}-$ $\left.q_{1}\right),\left(q_{3}-q_{2}\right), \ldots,\left(q_{N}-q_{N-1}\right)$, onde $q_{0}=0$, durante os tempos $t_{N}, t_{N}-t_{1}$, $t_{N}-t_{2}, \ldots, t_{N}-t_{N-1}$, respectivamente.

Ainda considerando que o reservatório é infinito e partindo da equação ( 40 ), a pressão em qualquer ponto do sistema é:

$$
p_{i}-p\left(r_{w}, t\right)=\Delta p(r, t)=\frac{\alpha_{p} q_{w} B_{w}}{h k \hat{\lambda}_{o}}\left[-\frac{1}{2} E_{i}\left(-\frac{r^{2} \phi \hat{c}_{t o}}{4 \alpha_{t} k \hat{\lambda}_{o} t}\right)\right]
$$

No caso em que a vazão é variável, como a EDP é linear, a solução é obtida a partir de um somatório das soluções de vazão constante, utilizando-se a superposição de efeitos. 


$$
\begin{aligned}
\Delta p\left(r, t_{N}\right)= & \frac{\alpha_{p} B}{h k \hat{\lambda}_{o}}\left\{q_{1}\left[-\frac{1}{2} E_{i}\left(-\frac{r^{2} \phi \hat{c}_{t o}}{4 \alpha_{t} k \hat{\lambda}_{o} t}\right)\right]\right. \\
& +\left(q_{2}-q_{1}\right)\left[-\frac{1}{2} E_{i}\left(-\frac{r^{2} \phi \hat{c}_{t o}}{4 \alpha_{t} k \hat{\lambda}_{o}\left(t-t_{1}\right)}\right)\right] \\
& +\left(q_{3}-q_{2}\right)\left[-\frac{1}{2} E_{i}\left(-\frac{r^{2} \phi \hat{c}_{t o}}{4 \alpha_{t} k \hat{\lambda}_{o}\left(t-t_{2}\right)}\right)\right]+\cdots \\
& \left.+\left(q_{n}-q_{n-1}\right)\left[-\frac{1}{2} E_{i}\left(-\frac{r^{2} \phi \hat{c}_{t o}}{4 \alpha_{t} k \hat{\lambda}_{o}\left(t-t_{n-1}\right)}\right)\right]\right\}
\end{aligned}
$$

Pode-se ainda escrever essa solução como um somatório, conforme indicado na equação ( 44 ).

$$
\Delta p\left(r, t_{N}\right)=\frac{\alpha_{p} B}{h k \hat{\lambda}_{o}} \sum_{j=1}^{n}\left(q_{j}-q_{j-1}\right)\left[-\frac{1}{2} E_{i}\left(-\frac{r^{2} \phi \hat{c}_{t o}}{4 \alpha_{t} k \hat{\lambda}_{o}\left(t-t_{j-1}\right)}\right)\right]
$$

Utilizando novamente a aproximação para tempos maiores que um segundo para $E_{i}$, encontra-se a solução analítica aproximada para o comportamento da pressão em escoamentos monofásicos com vazão de injeção variável:

$$
\Delta p\left(r, t_{N}\right)=\frac{\alpha_{p} B}{2 h k \hat{\lambda}_{o}} \sum_{j=1}^{n}\left(q_{j}-q_{j-1}\right) \ln \left(\frac{4 \alpha_{t} k \hat{\lambda}_{o}\left(t-t_{j-1}\right)}{e^{\gamma} \hat{c}_{t o} \phi r_{w}{ }^{2}}\right)
$$

Sabe-se que vazão é dada por:

$$
q(r, t)=\frac{h k \hat{\lambda}_{o}}{\alpha_{p} \mathrm{~B}} r \frac{\partial p}{\partial r}
$$

Substituindo a equação ( 44 ) em ( 46 ),

$$
q(r, t)=r_{w} \frac{\partial}{\partial r} \sum_{j=1}^{n}\left(q_{j}-q_{j-1}\right)\left[-\frac{1}{2} E_{i}\left(-\frac{r^{2} \phi \hat{c}_{t o}}{4 \alpha_{t} k \hat{\lambda}_{o}\left(t-t_{j-1}\right)}\right)\right]
$$


Logo, a vazão em cada instante para um poço produzindo com vazão variável pode ser escrita como:

$$
\begin{aligned}
& q(r, t) \\
& =r_{w} \sum_{j=1}^{n}\left[\left(q_{j}\right.\right. \\
& \left.\left.-q_{j-1}\right) \frac{1}{2} \frac{4 \alpha_{t} k \hat{\lambda}_{o}\left(t-t_{j-1}\right)}{r^{2} \phi \hat{c}_{t o}} e^{\left(-\frac{r^{2} \phi \hat{c}_{t o}}{4 \alpha_{t} k \hat{\lambda}_{o}\left(t-t_{j-1}\right)}\right)} \frac{2 r \phi \hat{c}_{t o}}{4 \alpha_{t} k \hat{\lambda}_{o}\left(t-t_{j-1}\right)}\right] \\
& q(r, t)=\sum_{j=1}^{n}\left(q_{j}-q_{j-1}\right) e^{-\left[\frac{c t \mu \phi \mathrm{r}^{2}}{4 \alpha_{t} k\left(t-t_{j-1}\right)}\right]}
\end{aligned}
$$




\section{3}

\section{Escoamento Bifásico: Períodos de Injeção e Falloff}

O escoamento bifásico isotérmico pode ser descrito como um sistema de equações diferenciais parciais que descrevem a conservação de massa de óleo e água. Quando as equações de conservação de massa são satisfeitas, a Lei de Darcy, que descreve as perdas de pressão em cada fase, também é satisfeita (THOMPSON, 1997).

Em um teste de injetividade, uma fase única (água) é injetada continuamente por um determinado período de tempo em uma zona saturada de óleo seguida por um período de falloff, quando o poço injetor é fechado.
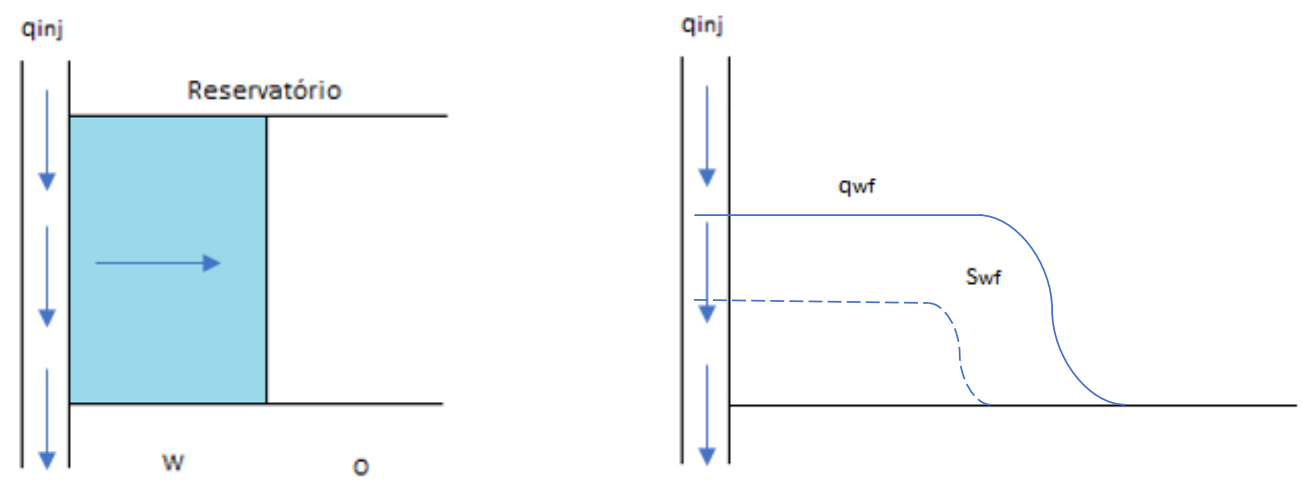

Figura 2- Injeção de água em um reservatório de óleo

Durante o período de injeção, ocorre um aumento na pressão do reservatório, enquanto no período de falloff ocorre uma queda na pressão do reservatório, conforme demostrado na Figura 3. 


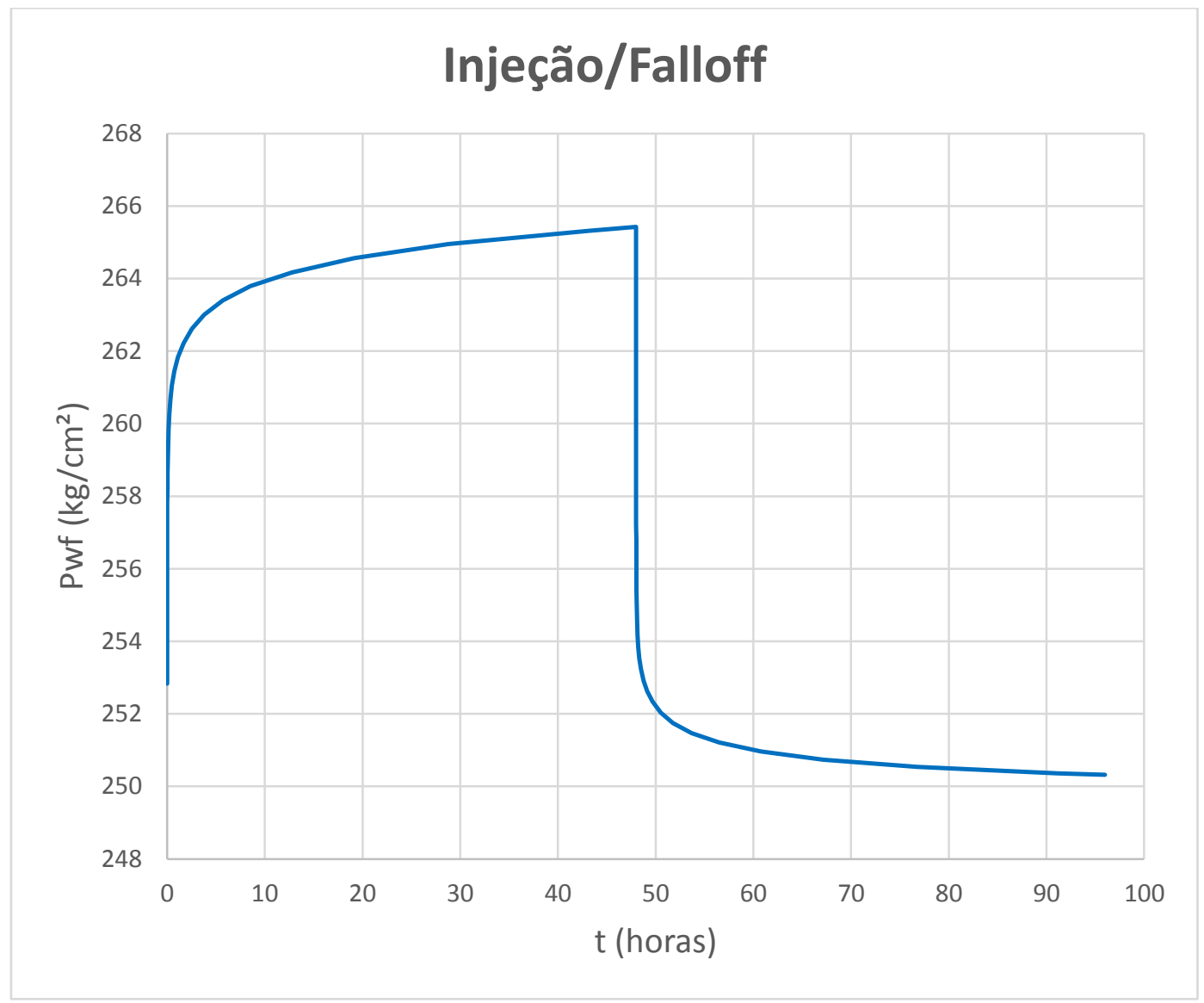

Figura 3 - Variação do comportamento da pressão

Nesse capítulo, primeiramente é apresentada a formulação para o período de injeção, e em seguida, a solução é desenvolvida para o período de falloff.

\section{1 .}

\section{Hipóteses do Modelo}

O modelo de reservatório utilizado dessa etapa do trabalho em diante, apresenta as características detalhadas abaixo. Além disso, do mesmo modo citado no capítulo anterior, o efeito gravitacional e a pressão capilar $\left(p_{c}\right)$ foram desprezados.

- Reservatório poroso homogêneo, isotrópico e radialmente infinito;

- Espessura constante 
- Escoamento bifásico (água-óleo)

- Escoamento imiscível

- Viscosidades constantes

- Escoamento isotérmico

- Poço radial

- Fluido flui perpendicularmente à área da seção do poço

- Reservatório infinito

- Pressão capilar $\left(p_{c}\right)=0$

- Grãos e fluidos pouco compressíveis

- Porosidade não se altera;

- Efeito gravitacional desprezado

- Pequenos gradientes de pressão

- Fluidos e rochas não reagentes entre si;

- Sistema inicialmente em equilíbrio;

- Não é considerado o dano à formação (skin).

\section{2.}

\section{Período de Injeção}

Primeiramente é desenvolvida a solução analítica para o comportamento da pressão de fundo de poço em escoamentos bifásicos durante o período de injeção de água em um reservatório (PERES;BOUGHRARA, 2006).

Da mesma forma que foi feito para o desenvolvimento da formulação para escoamentos monofásicos, parte-se da equação da continuidade, que descreve a conservação de massa:

$$
\frac{1}{r} \frac{\partial}{\partial r}\left(r \rho_{l} v_{l}\right)=-\frac{\partial}{\partial t}\left(\phi \rho_{l} S_{l}\right)
$$

Para casos bifásicos, segundo a Lei de Darcy, a velocidade aparente de cada fluido é dada por: 


$$
\begin{aligned}
& v_{o}(t, r)=\frac{-k_{o}}{\mu_{o}} \frac{\partial p_{o}}{\partial r} \\
& v_{w}(t, r)=\frac{-k_{w}}{\mu_{w}} \frac{\partial p_{w}}{\partial r}
\end{aligned}
$$

Onde $p_{o}=p_{c}-p_{w}$.

Nesse caso, consideramos a pressão capilar nula, de forma que $p_{o}=$ $p_{w}=p$, e, portanto:

$$
v_{w}(t, r)=\frac{-k_{w}}{\mu_{w}} \frac{\partial p}{\partial r}
$$

$$
v_{o}(t, r)=\frac{-k_{o}}{\mu_{o}} \frac{\partial p}{\partial r}
$$

Onde $k_{o}=k \cdot k_{r o}(S w)$, é a permeabilidade efetiva do óleo e $k_{r o}(S w)$ é a permeabilidade relativa do óleo e $k_{w}=k . k_{r w}(S w)$, é a permeabilidade efetiva da água e $k_{r w}(S w)$ é a permeabilidade relativa da água.

Um exemplo de distribuição das permeabilidades relativas é dado na Figura 4, onde $S_{w i}$ é a saturação de água inicial ou irredutível e $S_{o r}$ é a saturação de óleo residual. 


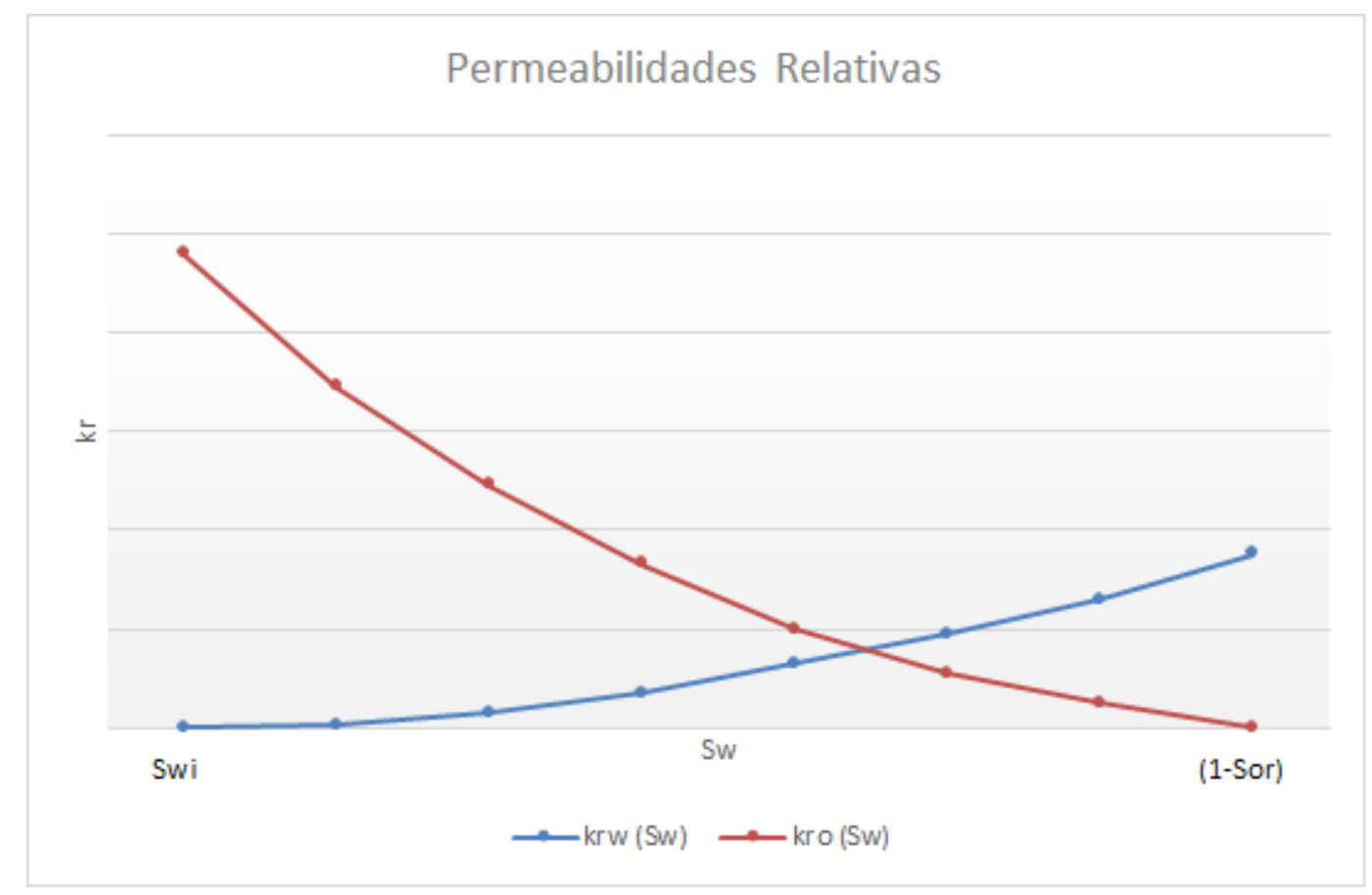

Figura 4 - Gráfico de Permeabilidades Relativas

A mobilidade da fase, nesse caso, é definida por:

$$
\lambda_{l}=\frac{k_{r l}}{\mu_{l}}
$$

Outro conceito muito utilizado é o da mobilidade do óleo no ponto de saturação de água irredutível, $S w_{i}$, quando a permeabilidade efetiva da água é nula.

$$
\hat{\lambda}_{o}=\frac{k_{r o}\left(S w_{i}\right)}{\mu_{o}}
$$

Similarmente, a mobilidade da água no ponto de saturação residual de óleo, 1 -Sor, quando a permeabilidade efetiva do óleo é nula é dada por: 


$$
\hat{\lambda}_{w}=\frac{k_{r w}(1-\text { Sor })}{\mu_{w}}
$$

Sabe-se ainda que a mobilidade total, $\lambda_{t}$, é dada por:

$$
\lambda_{t}(r, t)=\lambda_{w}(r, t)+\lambda_{o}(r, t)
$$

Com essas definições, a equação da continuidade pode ser escrita como:

$$
\frac{1}{r} \frac{\partial}{\partial r}\left(\rho_{l} \frac{k k_{r l}\left(S_{w}\right)}{\mu_{l}} r \frac{\partial p}{\partial r}\right)=\frac{\partial}{\partial t}\left(\phi \rho_{l} S_{l}\right)
$$

Pode-se expandir o lado esquerdo da equação ( 59 ) utilizando-se a derivada do produto e as equações da compressibilidade:

$$
\begin{array}{r}
\rho_{l}\left[\frac{1}{r} \frac{\partial}{\partial r}\left(\frac{k k_{r l}\left(S_{w}\right)}{\mu_{l}} r \frac{\partial p}{\partial r}\right)\right]+\left(\frac{k k_{r l}\left(S_{w}\right)}{\mu_{l}} \frac{\partial p}{\partial r} \frac{\partial \rho_{l}}{\partial r}\right) \\
=\rho_{l}\left[\frac{1}{r} \frac{\partial}{\partial r}\left(\frac{k k_{r l}\left(S_{w}\right)}{\mu_{l}} r \frac{\partial p}{\partial r}\right)+\left(\frac{k k_{r l}\left(S_{w}\right)}{\mu_{l}} c_{l}\left(\frac{\partial p}{\partial r}\right)^{2}\right)\right]
\end{array}
$$

Da mesma forma, a expansão do lado direito da equação ( 59 ) ( 3 ) pode ser escrita como:

$$
\frac{\partial}{\partial t}\left(\phi \rho_{l} S_{l}\right)=\phi \rho_{l} \frac{\partial S_{l}}{\partial t}+S_{l} \frac{\partial \phi \rho_{l}}{\partial t}=\phi \rho_{l} \frac{\partial S_{l}}{\partial t}+S_{l}\left[\phi \frac{\partial \rho_{l}}{\partial t}+\rho_{l} \frac{\partial \phi}{\partial t}\right]
$$

Utilizando as equações de compressibilidade ( 6 ) e ( 7 ) e as relações ( 8 ) e ( 9$)$ :

$$
\frac{\partial}{\partial t}\left(\phi \rho_{l} S_{l}\right)=\phi \rho_{l}\left[\frac{\partial S_{l}}{\partial t}+S_{l}\left(c_{l}+c_{r}\right) \frac{\partial p}{\partial t}\right]
$$


De forma que a equação ( 59 ) pode ser escrita como:

$$
\begin{aligned}
& \frac{1}{r} \frac{\partial}{\partial r}\left(\frac{k k_{r l}\left(S_{w}\right)}{\mu_{l}} r \frac{\partial p}{\partial r}\right)+\left(\frac{k k_{r l}\left(S_{w}\right)}{\mu_{l}} c_{l}\left(\frac{\partial p}{\partial r}\right)^{2}\right) \\
& =\phi\left[\frac{\partial S_{l}}{\partial t}+S_{l}\left(c_{l}+c_{r}\right) \frac{\partial p}{\partial t}\right]
\end{aligned}
$$

Somando as EDPs de cada fase, elimina-se a derivada parcial da saturação, de forma que:

$$
\begin{aligned}
\frac{1}{r} \frac{\partial}{\partial r}\left(k\left(\frac{k_{r o}\left(S_{w}\right)}{\mu_{o}}+\frac{k_{r o}\left(S_{w}\right)}{\mu_{w}}\right) r \frac{\partial p}{\partial r}\right) \\
+k\left[\frac{k_{r o}\left(S_{w}\right)}{\mu_{o}} c_{o}+\frac{k_{r w}\left(S_{w}\right)}{\mu_{w}} c_{w}\right]\left(\frac{\partial p}{\partial r}\right)^{2} \\
=\phi\left[S_{o} c_{o}+S_{w} c_{w}+c_{r}\right] \frac{\partial p}{\partial t}
\end{aligned}
$$

A compressibilidade total é dada por:

$$
c_{w}=S_{o} c_{o}+S_{w} c_{w}+c_{r}
$$

Sendo assim, a equação ( 64 ) pode ser reescrita como:

$$
\frac{1}{r} \frac{\partial}{\partial r}\left(k \lambda_{t} r \frac{\partial p}{\partial r}\right)+k\left[\lambda_{o} c_{o}+\lambda_{w} c_{w}\right]\left(\frac{\partial p}{\partial r}\right)^{2}=\phi c_{t} \frac{\partial p}{\partial t}
$$

Desprezando-se o termo quadrático pode-se escrever:

$$
\frac{1}{r} \frac{\partial}{\partial r}\left(k \lambda_{t} r \frac{\partial p}{\partial r}\right)=\phi c_{t} \frac{\partial p}{\partial t}
$$

No caso em estudo, onde considera-se um reservatório homogêneo e viscosidades constantes: 
$\frac{1}{r} \frac{\partial}{\partial r}\left(k r \frac{\partial p}{\partial r}\right)=\phi \frac{c_{t}}{\lambda_{t}} \frac{\partial p}{\partial t}$

Para obter a solução para um caso particular é necessário especificar as condições de contorno.

Nas hipóteses assumidas, o sistema é composto pelas seguintes equações:

$$
\begin{array}{lc}
\text { EDP } & \frac{1}{r} \frac{\partial}{\partial r}\left(r \frac{\partial p}{\partial r}\right)=\frac{\phi}{k} \frac{c_{t}}{\lambda_{t}} \frac{\partial p}{\partial t} \\
\text { Cl } & p(r, t=0)=p_{i} \\
\text { CCE } & \lim _{\infty} p(r, t)=p_{i} \\
\mathrm{CCl} & -2 \pi k h\left(\lambda_{t} r \frac{\partial p}{\partial r}\right)_{r_{w}}=q_{i n j} B_{w}
\end{array}
$$

Da mesma forma que foi demonstrado para o caso monofásico, a solução desse problema também pode ser desenvolvida partindo-se diretamente da vazão de injeção, $q_{i n j}$, dada pela soma das vazões da água e do óleo:

$$
q_{i n j}=q_{w}+q_{o}
$$

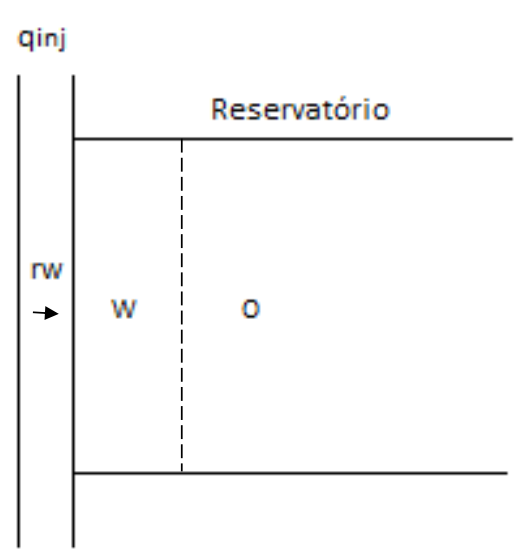

Figura 5 - Representação da injeção de água no reservatório 
Conforme visto no anteriormente, as vazões da água e do óleo são, respectivamente:

$$
\begin{aligned}
& q_{w}(r, t)=\frac{2 \pi h r k_{w}}{\mu_{w}} \frac{\partial p}{\partial r} \\
& q_{o}(r, t)=\frac{2 \pi h r k_{o}}{\mu_{o}} \frac{\partial p}{\partial r}
\end{aligned}
$$

Logo,

$$
q_{i n j}=\frac{2 \pi h r k_{w}}{\mu_{w}} \frac{\partial p}{\partial r}+\frac{2 \pi h r k_{o}}{\mu_{o}} \frac{\partial p}{\partial r}
$$

$$
q_{i n j}=2 \pi h\left(\frac{k_{w}}{\mu_{w}}+\frac{k_{o}}{\mu_{o}}\right) r \frac{\partial p}{\partial r}
$$

Utilizando a definição de mobilidade, pode-se escrever:

$$
q_{i n j}(r, t)=2 \pi h k(r)\left(\lambda_{w}(r, t)+\lambda_{o}(r, t)\right) r \frac{\partial p(r, t)}{\partial r}
$$

A equação pode ser escrita ainda em função da mobilidade total.

$$
q_{i n j}(r, t)=2 \pi h k(r) \lambda_{t}(r, t) r \frac{\partial p(r, t)}{\partial r}
$$

Rearranjando os termos da equação:

$$
\frac{\partial p(r, t)}{\partial r}=\frac{q_{i n j}(r, t)}{2 \pi h k(r)} \frac{1}{\lambda_{t}(r, t)} \frac{1}{r}
$$

Para reservatórios infinitos, integrando do raio do poço $\left(r_{w}\right)$ até $\infty$ : 


$$
\int_{r_{w}}^{\infty} \frac{\partial p(r, t)}{\partial r} d r=\frac{1}{2 \pi h} \int_{r_{w}}^{\infty} \frac{q_{i n j}(r, t)}{r k(r) \lambda_{t}(r, t)} d r
$$

A permeabilidade é constante no caso de reservatórios isotrópicos, de forma que:

$$
p_{w f}-p_{i}=\frac{1}{2 \pi h k} \int_{r_{w}}^{\infty} \frac{q_{i n j}(r, t)}{r \lambda_{t}(r, t)} d r
$$

Pode-se ainda dividir a equação ( 82 ) em dois períodos, representando o fluxo antes e depois da posição da frente de avanço de água, $r_{f}(t)$.

$$
p_{w f}-p_{i}=\frac{1}{2 \pi h k}\left[\int_{r_{w}}^{r_{f}(t)} \frac{q_{i n j}(r, t)}{r \lambda_{t}(r, t)} d r+\int_{r_{f}(t)}^{\infty} \frac{q_{i n j}(r, t)}{r \lambda_{t}(r, t)} d r\right]
$$

Onde $r_{f}(t)$ pode ser obtida através da equação de Buckley-Leverett (1942), que conforme demonstrado no Apêndice A é:

$$
r\left(S_{w}\right)=\sqrt{r^{2}{ }_{w}+\frac{q B_{w}}{24 \pi h \phi} f_{w}{ }^{\prime}\left(S_{w}\right) t}
$$

A Figura 6 representa esquematicamente a frente de avanço de água no reservatório ao longo do tempo. 


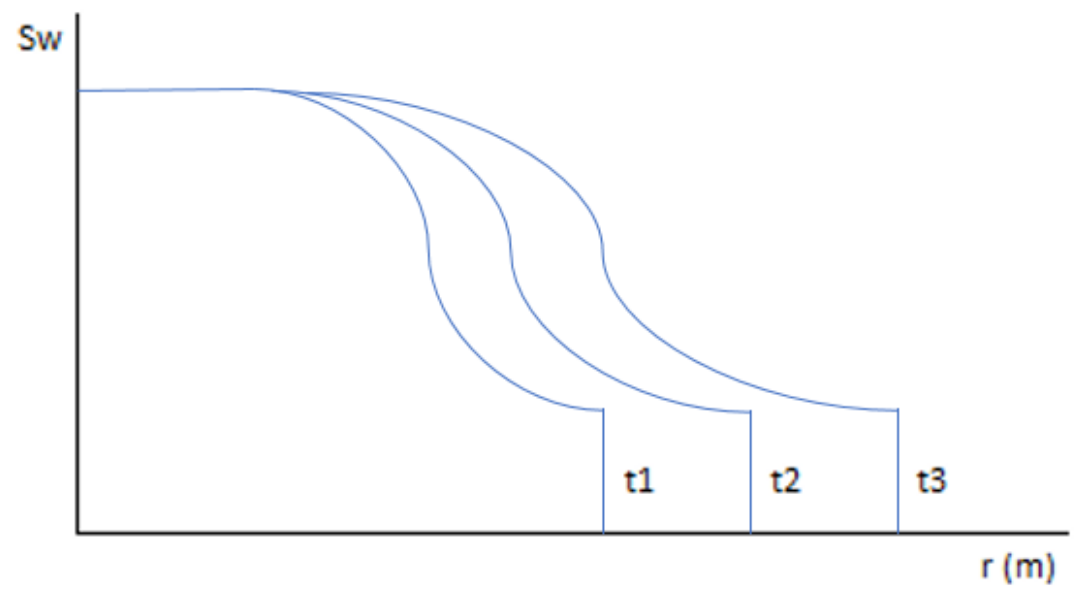

Figura 6 - Variação da posição da frente de avanço de água

Após a posição da frente de avanço de água, observa-se que o reservatório está saturado apenas com óleo, de forma que $q_{t}(r, t)=\hat{q}_{o}(r, t)$ e $\lambda_{t}(r, t)=\hat{\lambda}_{o}$. Com essas considerações, pode-se escrever a equação ( 83 ) como:

$$
p_{w f}-p_{i}=\frac{1}{2 \pi h k}\left[\int_{r_{w}}^{r_{f}(t)} \frac{q_{i n j}(r, t)}{r \lambda_{t}(r, t)} d r+\int_{r_{f}(t)}^{\infty} \frac{\hat{q}_{o}(r, t)}{r \hat{\lambda}_{o}} d r\right]
$$

Somando e subtraindo o termo $\int_{r_{w}}^{r_{f}(t)} \frac{\hat{a}_{o}(r, t)}{r \hat{\lambda}_{o}} d r$ ao termo entre colchetes:

$$
\begin{gathered}
p_{w f}-p_{i}=\frac{1}{2 \pi h k}\left[\int_{r_{w}}^{r_{f}(t)} \frac{q_{i n j}(r, t)}{r \lambda_{t}(r, t)} d r-\int_{r_{w}}^{r_{f}(t)} \frac{\hat{q}_{o}(r, t)}{r \hat{\lambda}_{o}} d r\right. \\
\left.+\int_{r_{f}(t)}^{\infty} \frac{\hat{q}_{o}(r, t)}{r \hat{\lambda}_{o}} d r+\int_{r_{w}}^{r_{f}(t)} \frac{\hat{q}_{o}(r, t)}{r \hat{\lambda}_{o}} d r\right]
\end{gathered}
$$




$$
\begin{gathered}
p_{w f}-p_{i}=\frac{1}{2 \pi h k}\left[\left\{\int_{r_{w}}^{r_{f}(t)} \frac{q_{i n j}(r, t)}{r \lambda_{t}(r, t)} d r-\int_{r_{w}}^{r_{f}(t)} \frac{\hat{q}_{o}(r, t)}{r \hat{\lambda}_{o}} d r\right\}\right. \\
\left.+\frac{1}{2 \pi h k}\left\{\int_{r_{w}}^{\infty} \frac{\hat{q}_{o}(r, t)}{r \hat{\lambda}_{o}} d r\right\}\right]
\end{gathered}
$$

Entretanto, para $r<r_{f}(t)$, a vazão $\hat{q}_{o}(r, t)=q_{i n j}(r, t)=q_{w} B_{w}$, logo:

$$
\begin{aligned}
p_{w f}-p_{i}=\frac{1}{2 \pi h k \hat{\lambda}_{o}} & {\left[\left\{\int_{r_{w}}^{\infty} \frac{\hat{q}_{o}(r, t)}{r} d r\right\}\right.} \\
& \left.+\frac{q_{w} B_{w}}{2 \pi h k \hat{\lambda}_{o}}\left\{\int_{r_{w}}^{r_{f}(t)}\left(\frac{\hat{\lambda}_{o}}{\lambda_{t}(r, t)}-1\right) \frac{d r}{r}\right\}\right]
\end{aligned}
$$

Utilizando a constante $\alpha_{p}$ para adequação de unidades ao sistema de medidas da Brasileiro, conforme indicado na Tabela 1, podemos escrever:

$$
\begin{aligned}
p_{w f}-p_{i}=\frac{\alpha_{p}}{h k \hat{\lambda}_{o}} & {\left[\left\{\int_{r_{w}}^{\infty} \frac{\hat{q}_{o}(r, t)}{r} d r\right\}\right.} \\
+ & \left.\frac{\alpha_{p} q_{w} B_{w}}{h k \hat{\lambda}_{o}}\left\{\int_{r_{w}}^{r_{f}(t)}\left(\frac{\hat{\lambda}_{o}}{\lambda_{t}(r, t)}-1\right) \frac{d r}{r}\right\}\right]
\end{aligned}
$$

Onde $\frac{\alpha_{p}}{h k \widehat{\lambda}_{o}}\left[\int_{r_{w}}^{\infty} \frac{\hat{q}_{o}(r, t)}{r} d r\right]=\Delta \widehat{p_{o}}$, que representa a solução monofásica calculada com propriedades da fase óleo $@ S_{w i}$ (na saturação inicial de água).

Utilizando a aproximação logarítmica para o período de injeção:

$$
\Delta \widehat{p_{o}}(\mathrm{t})=\frac{\alpha_{p} q_{w} B_{w}}{h k \hat{\lambda}_{o}}\left[\frac{1}{2} \ln \left(\frac{4 \alpha_{t} k \hat{\lambda}_{o} t}{e^{\gamma} \widehat{c_{t o}} \phi r_{w}^{2}}\right)\right]
$$

Substituindo a equação ( 90 ) na equação

( 89 ), encontra-se a solução analítica aproximada para o comportamento da pressão durante o período de injeção de água: 


$$
\begin{aligned}
p_{w f}-p_{i}=\frac{\alpha_{p} q_{w} B_{w}}{2 h k \hat{\lambda}_{o}}\left[\left\{\ln \left(\frac{4 \alpha_{t} k \hat{\lambda}_{o} t}{e^{\gamma} \widehat{c_{t o}} \phi r_{w}^{2}}\right)\right\}\right. \\
\left.+\frac{\alpha_{p} q_{w} B_{w}}{h k \hat{\lambda}_{o}}\left\{\int_{r_{w}}^{r_{f}(t)}\left(\frac{\hat{\lambda}_{o}}{\lambda_{t}\left(r^{\prime}, t\right)}-1\right) \frac{d r^{\prime}}{r^{\prime}}\right\}\right]
\end{aligned}
$$

Usualmente representa-se a equação acima por:

$$
\Delta p(t)=\Delta \widehat{p_{o}}(t)+\Delta p_{\lambda}(t)
$$

Onde $\Delta \widehat{p_{o}}$ representa a solução monofásica para o período de injeção e $\Delta p_{\lambda}$ é a queda de pressão adicional devido ao contraste na mobilidade das fases óleo e água.

\subsection{1.}

\section{Período de Injeção com Deslocamento Pistão}

Aqui, é desenvolvida a solução para o caso específico de injeção de água em um reservatório de óleo, onde a frente de avanço da água se move como se fosse o deslocamento de um pistão, muito utilizado para auxiliar a compreensão de problemas de escoamento de fluidos.

A Figura 7 traz uma representação esquemática do deslocamento pistão em regime permanente.

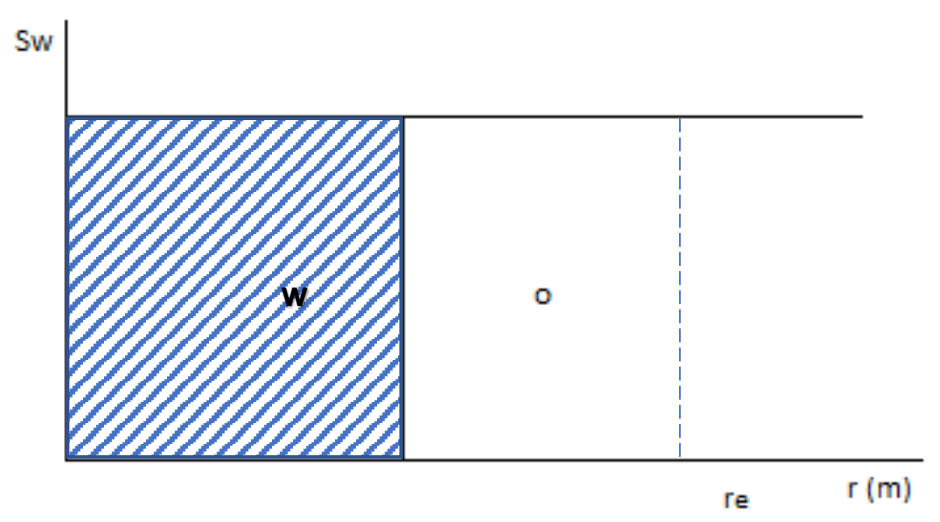

Figura 7 - Deslocamento em pistão 
Nesse caso, do raio da frente de avanço de água ao raio externo do reservatório, o único fluido presente é o óleo, de forma que é possível escrever:

$$
p_{e}-p_{w f}=\frac{q_{i n j}}{h \hat{\lambda}_{o}} \int_{r_{w}}^{r f(t)}\left[\frac{\hat{\lambda}_{o}}{\lambda_{t}}-1\right] \frac{1}{r} d r+\frac{q_{i n j}}{2 h \hat{\lambda}_{o}} \ln \left(\frac{r_{e}}{r_{w}}\right)
$$

Além disso, nesse caso, $\lambda_{t}=\lambda_{w}$, pois até o raio da frente de avanço, o único fluido presente é a água, de forma que:

$$
\begin{aligned}
& \hat{\lambda}_{o}=\lambda_{o} @ S_{o i} \\
& \hat{\lambda}_{w}=\lambda_{w} @\left(1-S_{o r}\right) \\
& \lambda_{t}=\hat{\lambda}_{w}
\end{aligned}
$$

Sendo assim, a equação ( 93 ) pode ser rescrita como:

$$
p_{e}-p_{w f}=\frac{q_{i n j}}{h \hat{\lambda}_{o}} \int_{r_{w}}^{r f(t)}\left[\frac{\hat{\lambda}_{o}}{\hat{\lambda}_{w}}-1\right] \frac{1}{r} d r+\frac{q_{i n j}}{2 h \hat{\lambda}_{o}} \ln \left(\frac{r_{e}}{r_{w}}\right)
$$

A razão entre as mobilidades da água e do óleo em um reservatório de óleo submetido à injeção de água pode ser um indicativo de como será o escoamento, como por exemplo, se haverá fingers de um fluido no outro.

$$
M=\frac{\lambda_{w}}{\lambda_{o}}
$$

Pode-se escrever ainda:

$$
\widehat{M}=\frac{\hat{\lambda}_{w}}{\hat{\lambda}_{o}}
$$

De forma que a equação ( 97 ) se torna: 


$$
p_{e}-p_{w f}=\frac{q_{i n j}}{h \hat{\lambda}_{o}}\left[\left(\frac{1-\widehat{M}}{\widehat{M}}\right) \ln \left(\frac{r_{f(t)}}{r_{w}}\right)+\frac{1}{2} \ln \left(\frac{r_{e}}{r_{w}}\right)\right]
$$

Observa-se que para valores de $\widehat{M}>1$, o termo relativo ao contraste de mobilidade, $\Delta p_{\lambda}$, é negativo, e para valores de $\widehat{M}<1$, o termo relativo ao contraste de mobilidade, $\Delta p_{\lambda}$, é positivo.

\section{3.}

\section{Período de Falloff}

Nesse item, é desenvolvida a solução analítica para o comportamento da pressão de fundo de poço em escoamentos bifásicos durante o período de período de falloff, quando o poço injetor é fechado, de forma que a vazão é constante e igual a zero (PERES;BOUGHRARA, 2006). Nesse caso, assume-se que a frente de água é estacionária após o fechamento do poço injetor, conforme indicado na Figura 8, o que é comprovado por meio de simulações numéricas quando são considerados os tempos de testes de formação aplicados na indústria.
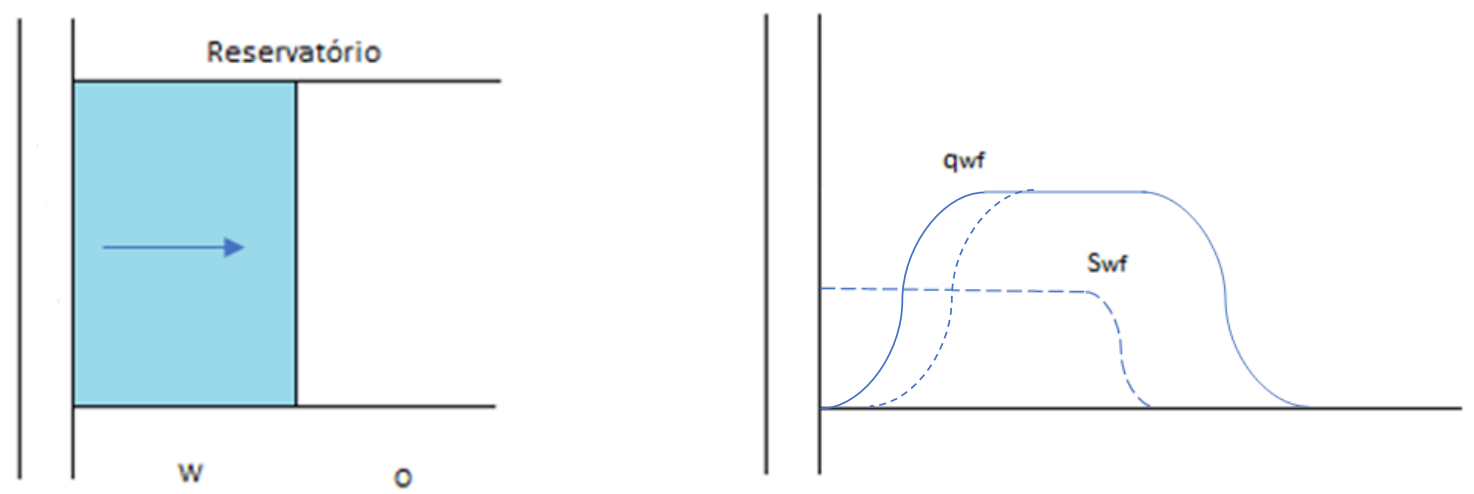

Figura 8 - Representação do período de falloff

Seguindo os mesmos passos que foram realizados para encontrar a solução para o período de injeção, partindo da Lei de Darcy, encontra-se a 
equação ( 101 ), onde s representa o período de estática ( ou de fechamento), sem fluxo.

$$
p_{w s}(\Delta t)-p_{i}=\Delta p_{s}=\frac{\alpha}{h k} \int_{r_{w}}^{\infty} \frac{q_{t s}(r, \Delta t)}{r \lambda_{t}(r, \Delta t)} d r
$$

Dado, então, que a frente de avanço é estacionária, tem-se que o perfil de mobilidade não se altera durante o período de falloff, de forma que:

$$
\Delta p_{s}=\frac{\alpha}{h k} \int_{r_{w}}^{\infty} \frac{q_{t s}(r, \Delta t)}{r \lambda_{t}\left(r, t_{p}\right)} d r
$$

Essa equação pode ser separada em duas integrais, do raio do poço à posição da frente de avanço de água, e dessa frente em diante.

$$
\Delta p_{s}=\frac{\alpha}{h k}\left[\int_{r_{w}}^{r_{f}(t p)} \frac{q_{t s}(r, \Delta t)}{r \lambda_{t}\left(r, t_{p}\right)} d r+\int_{r_{f}(t p)}^{\infty} \frac{q_{t s}(r, \Delta t)}{r \lambda_{t}\left(r, t_{p}\right)} d r\right]
$$

Da frente de avanço de água em diante o reservatório está saturado apenas com óleo.

$$
\Delta p_{s}=\frac{\alpha}{h k}\left[\int_{r_{f}(t p)}^{\infty} \frac{q_{t s}(r, \Delta t)}{r \hat{\lambda}_{o}} d r+\int_{r_{w}}^{r_{f}(t p)} \frac{q_{t s}(r, \Delta t)}{r \lambda_{t}\left(r, t_{p}\right)} d r\right]
$$

sabe-se que $q_{t s}(r, \Delta t)=\hat{q}_{o s}(r, \Delta t)$, após a frente de avanço de água, ou seja $r>r_{f}(t p)$, logo:

$$
\Delta p_{s}(\Delta t)=\frac{\alpha}{h k \hat{\lambda}_{o}} \int_{r_{f}(t p)}^{\infty} \frac{\hat{q}_{o s}(r, \Delta t)}{r} d \mathrm{r}+\frac{\alpha}{h k} \int_{r_{w}}^{r_{f}(t p)} \frac{q_{t s}(\mathrm{r}, \Delta t)}{r^{\prime} \lambda_{t}\left(\mathrm{r}, t_{p}\right)} \frac{d \mathrm{r}}{r}
$$

Adicionando e subtraindo o termo $\frac{\alpha}{h k} \int_{r_{w}}^{r_{f}(t)} \frac{\hat{q}_{o s}(r, \Delta t)}{r \widehat{\lambda}_{o}} d r$ ao lado direito da equação: 


$$
\begin{aligned}
\Delta p_{s}(\Delta t)=\frac{\alpha}{h k \hat{\lambda}_{o}} & \int_{r_{w}}^{\infty} \frac{\hat{q}_{o s}(r, \Delta t)}{r} d r \\
& +\frac{\alpha}{h k} \int_{r_{w}}^{r_{f}(t p)}\left\{\frac{q_{t s}(r, \Delta t)}{r \lambda_{t}\left(r, t_{p}\right)}-\frac{\hat{q}_{o s}(r, \Delta t)}{r \hat{\lambda}_{o}}\right\} \frac{d r}{r}
\end{aligned}
$$

Em analogia à representação do período de injeção, pode-se escrever:

$$
\Delta p_{s}(\Delta t)=\Delta \hat{p}_{o s}(\Delta t)+\Delta p_{\lambda}(\Delta t)
$$

A solução para o período de falloff, $\Delta p_{s}(\Delta t)$, pode ser descrita como a soma da solução monofásica “óleo" @ $S_{w i}$ do período de estática, $\Delta \widehat{p_{o s}}(\Delta t)$, e a variação de pressão devido ao contraste na mobilidade das fases óleo e água $\Delta p_{\lambda}(\Delta t)$.

A solução monofásica "óleo" @ $S_{w i}$ do período de estática pode ainda ser escrita utilizando o princípio da superposição, Nesse caso, considerase que a pressão durante a estática pode ser representada pela superposição dos efeitos da continuidade da injeção no poço e de um poço produtor na mesma localização, conforme pode ser observado na Figura 9. Dessa forma, a solução monofásica “óleo" @ $S_{w i}$ da estática é dada por: 
$\Delta \hat{p}_{o s}(\Delta t)=\Delta \hat{p}_{o s}\left(t_{p}+\Delta t\right)-\Delta \hat{p}_{o s}(\Delta t)$

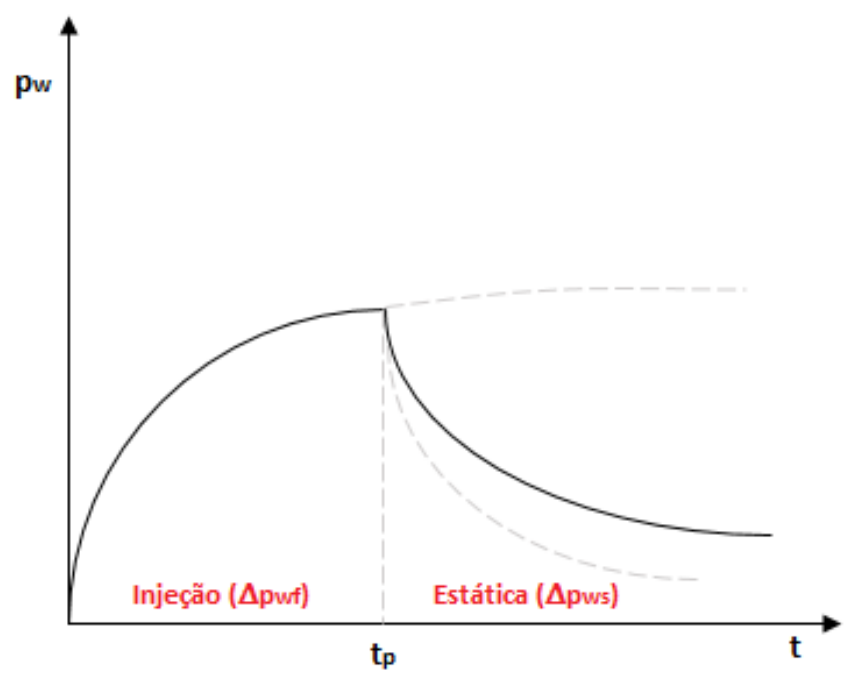

Figura 9 - Representação da solução no período de estática

Expandindo a equação ( 108 ),

$$
\begin{array}{r}
\Delta \hat{p}_{o s}(\Delta t)=\frac{q_{i n j} B \alpha_{p}}{2 k h \hat{\lambda}_{o}} \ln \left(\frac{4 \alpha_{p} \hat{\lambda}_{o} k}{e^{\gamma} c_{t} \phi r_{w}{ }^{2}}\left(t_{p}+\Delta t\right)\right) \\
-\frac{q_{i n j} B \alpha_{p}}{2 k h \hat{\lambda}_{o}} \ln \left(\frac{4 \alpha_{p} \hat{\lambda}_{o} k}{e^{\gamma} c_{t} \phi r_{w}{ }^{2}}(\Delta t)\right)
\end{array}
$$

$\Delta \hat{p}_{o s}(\Delta t)=\frac{q_{i n j} B \alpha_{p}}{2 k h \hat{\lambda}_{o}} \ln \left(\frac{t_{p}+\Delta t}{\Delta t}\right)$

Substituindo na equação ( 107 ):

$$
\begin{aligned}
\Delta p_{s}(\Delta t)=\frac{q_{i n j} B \alpha_{p}}{2 k h \hat{\lambda}_{o}} & \ln \left(\frac{t_{p}+\Delta t}{\Delta t}\right) \\
& +\frac{\alpha}{h k} \int_{r_{w}}^{r_{f}(t p)}\left\{\frac{q_{t s}(r, \Delta t)}{\lambda_{t}\left(r, t_{p}\right)}-\frac{\hat{q}_{o s}(r, \Delta t)}{\hat{\lambda}_{o}}\right\} \frac{d r}{r}
\end{aligned}
$$

Como solução para a equação ( 111 ), pode-se utilizar algumas aproximações para descrever o perfil de vazões, $q_{t s}\left(r^{\prime}, \Delta t\right)$, dentre elas:

1. $\quad q_{t s}(r, \Delta t)=\hat{q}_{o s}(r, \Delta t)$ 
2.

3. $\quad q_{t s}(r, \Delta t)=\frac{\hat{q}_{o s}(r, \Delta t) \widehat{\lambda}_{o}+\hat{q}_{w s}(r, \Delta t) \widehat{\lambda}_{w}}{\widehat{\lambda}_{o}+\widehat{\lambda}_{w}}$

4. $\quad q_{t s}(r, \Delta t)=\frac{\hat{q}_{o s}(r, \Delta t) \widehat{\lambda}_{o}+\hat{q}_{w s}(r, \Delta t) \widehat{\lambda}_{w}}{\widehat{\lambda}_{o}(r)+\widehat{\lambda}_{w}(r)}$

5. $\quad q_{t s}(r, \Delta t)=\hat{q}_{\lambda s}\left(r, \Delta t, \lambda_{t}\left(r, t_{p}\right), c_{t}\left(r, t_{p}\right)\right)$

Nesse estudo, buscando simplificar as deduções, utiliza-se a aproximação $1, q_{t s}\left(r^{\prime}, \Delta t\right)=\widehat{q_{o s}}\left(r^{\prime}, \Delta t\right)$, de forma que:

$$
\begin{aligned}
\Delta p_{s}(\Delta t)=\frac{q_{\text {inj }} B \alpha_{p}}{2 k h \hat{\lambda}_{o}} & \ln \left(\frac{t_{p}+\Delta t}{\Delta t}\right) \\
& +\frac{\alpha}{h k} \int_{r_{w}}^{r_{f}(t p)}\left\{\frac{\hat{q}_{o s}(r, \Delta t)}{\lambda_{t}\left(r, t_{p}\right)}-\frac{\hat{q}_{o s}(r, \Delta t)}{\hat{\lambda}_{o}}\right\} \frac{d r}{r}
\end{aligned}
$$

Portanto, a solução analítica aproximada para o comportamento da pressão de fundo de poço durante o período de falloff é:

$$
\begin{aligned}
\Delta p_{s}(\Delta t)=\frac{q_{i n j} B \alpha_{p}}{2 k h \hat{\lambda}_{o}} & \ln \left(\frac{t_{p}+\Delta t}{\Delta t}\right) \\
& \quad+\frac{\alpha}{h k \hat{\lambda}_{o}} \int_{r_{w}}^{r_{f}(t p)}\left\{\frac{\hat{q}_{o s}(r, \Delta t) \hat{\lambda}_{o}}{\lambda_{t}\left(r, t_{p}\right)}-\hat{q}_{o s}(r, \Delta t)\right\} \frac{d r}{r}
\end{aligned}
$$

Para determinar o valor da vazão $\hat{q}_{o s}(r, \Delta t)$, utilizaremos os termos adimensionais (Apêndice $\mathrm{C}$ ): 
$q_{D}=\frac{q}{q_{\text {inj }}}$

$r_{D}=\frac{r}{r_{w}}$

$t_{D}=\frac{\alpha_{p} \hat{\lambda}_{o} k t}{c_{t o} \phi r_{w}^{2}}$

$t_{p D}=\frac{\alpha_{p} \hat{\lambda}_{o} k t_{p}}{c_{t o} \phi r_{w}^{2}}$

$p_{D}\left(r_{D}, t_{D}\right)=-\frac{1}{2} E_{i}\left(-\frac{r_{D}^{2}}{4 t_{D}}\right)$

Sabe-se que vazão $q_{D}$ no período de injeção é dada por:

$q_{D}\left(r_{D}, t_{D}\right)=r_{D} \frac{\partial p_{D}\left(r_{D}, t_{D}\right)}{\partial r_{D}}=e^{\left(-\frac{r_{D}^{2}}{4 \Delta t}\right)}$

$$
q_{D}\left(r_{D}, t_{D}\right)=r_{D} \frac{\partial}{\partial r_{D}}\left(-\frac{1}{2} E_{i}\left(-\frac{r_{D}^{2}}{4 t_{D}}\right)\right)
$$

A função exponencial, nesse caso, pode ser escrita como:

$$
-\frac{1}{2} E_{i}\left(-\frac{r_{D}^{2}}{4 t_{D}}\right)=-\frac{1}{2} \int_{\frac{r_{D}}{4 t_{D}}}^{\infty} \frac{e^{-u}}{u} d u
$$

Logo,

$$
\begin{aligned}
& q_{D}\left(r_{D}, t_{D}\right)=r_{D}\left(-\frac{1}{2}\right) \frac{4 t_{D}}{r_{D}{ }^{2}} e^{\left(-\frac{r_{D}^{2}}{4 t_{D}}\right)}\left(-\frac{2 r_{D}}{4 t_{D}}\right) \\
& q_{D}\left(r_{D}, t_{D}\right)=e^{\left(-\frac{r_{D}^{2}}{4 t_{D}}\right)}
\end{aligned}
$$

No período de falloff, a vazão pode ser interpretada como superposição da vazão do poço injetor e a de uma poço produtor com a 
mesma vazão na mesma localização, de forma que a equação para a vazão em um determinado instante é dada pela equação ( 124 ).

$$
\begin{aligned}
& q_{D s}\left(r_{D}, \Delta t\right)=q_{D}\left(r_{D}, t_{p}+\Delta t\right)-q_{D}\left(r_{D}, \Delta t\right) \\
& q_{D s}\left(r_{D}, \Delta t\right)=e^{\left(-\frac{r_{D}^{2}}{4\left(t_{p}+\Delta t\right)}\right)}-e^{\left(-\frac{r_{D}^{2}}{4 \Delta t}\right)}
\end{aligned}
$$

Além disso,

$$
q_{D}=\frac{q_{o}}{q_{\text {inj }}}
$$

Logo, em termos dimensionais a vazão em cada instante é:

$$
\widehat{q_{o s}}\left(r^{\prime}, \Delta t\right)=q_{i n j}\left(e^{\left(-\frac{c_{t o} \phi r^{2}}{4 \alpha_{p} \widehat{\lambda}_{o} k\left(t_{p}+\Delta t\right)}\right)}-e^{\left(-\frac{c_{t o} \phi r^{2}}{4 \alpha_{p} \bar{\lambda}_{o} k(\Delta t)}\right)}\right)
$$


4

\section{Escoamento Bifásico: Vazão Variável}

Com base nos estudos dos capítulos anteriores e no desenvolvimento das soluções analíticas para os períodos de injeção e falloff, nesse capítulo é proposta a solução analítica aproximada para o comportamento da pressão de fundo de poço no caso de um reservatório com um poço injetor com um esquema de vazão variável.

A equação ( 87 ), representa o comportamento da pressão de fundo de poço com o tempo para um caso geral de escoamento bifásico.

$$
\begin{gathered}
p_{w f}-p_{i}=\frac{1}{2 \pi h k}\left[\left\{\int_{r_{w}}^{r_{f}(t)} \frac{q_{i n j}(r, t)}{r^{\prime} \lambda_{t}(r, t)} d r-\int_{r_{w}}^{r_{f}(t)} \frac{\hat{q}_{o}(r, t)}{r \hat{\lambda}_{o}} d r\right\}\right. \\
\left.+\frac{1}{2 \pi h k}\left\{\int_{r_{w}}^{\infty} \frac{\hat{q}_{o}(r, t)}{r \hat{\lambda}_{o}} d r\right\}\right]
\end{gathered}
$$

Entretanto, para o caso em questão, onde a vazão varia com o tempo, o volume total de água injetada no reservatório $v_{i n j}$, em determinado instante é:

$$
v_{i n j}(t)=\int_{0}^{t} q_{i n j}(t) d t
$$

Sendo assim, é possível determinar a posição da frente de avanço, $r_{f}(t)$, a cada instante, por meio da equação o de Buckley-Leverett (1942) da seguinte forma: 


$$
r_{f}(t)=\sqrt{r^{2}{ }_{w}+\frac{B_{w}}{24 \pi h \phi} f_{w}{ }^{\prime}\left(S_{w}\right) \int_{0}^{t} q_{i n j}(t) d t}
$$

Nesse caso, para $r<r_{f}(t)$, a vazão no reservatório $\hat{q}_{o}(r, t)=$ $q_{\text {inj }}(r, t)$. Sendo que $q_{i n j}(r, t)$ pode ser aproximada pela superposição de vazões fornecida pela equação ( 48 ):

$$
q(r, t)=\sum_{j=1}^{n}\left(q_{j}-q_{j-1}\right) e^{-\left[\frac{c_{t} \mu \phi \mathrm{r}^{2}}{4 \alpha_{t} k\left(t_{j}-t_{j-1}\right)}\right]}
$$

Logo, a equação ( 128 ) pode ser escrita como:

$$
\begin{aligned}
p_{w f}-p_{i}=\frac{1}{2 \pi h k \hat{\lambda}_{o}} & {\left[\int_{r_{w}}^{\infty} \frac{\hat{q}_{o}(r, t)}{r} d r\right] } \\
& +\frac{1}{2 \pi h k \hat{\lambda}_{o}}\left[\int_{r_{w}}^{r_{f}(t)} q_{i n j}(r, t)\left(\frac{\hat{\lambda}_{o}}{\lambda_{t}(r, t)}-1\right) \frac{d r}{r}\right]
\end{aligned}
$$

Utilizando a constante $\alpha_{p}$ para adequação de unidades ao sistema de medidas da Brasileiro, conforme indicado na Tabela 1, podemos escrever ainda:

$$
\begin{aligned}
p_{w f}-p_{i}=\frac{\alpha_{p}}{h k \hat{\lambda}_{o}} & {\left[\left\{\int_{r_{w}}^{\infty} \frac{\hat{q}_{o}(r, t)}{r} d r\right\}\right.} \\
+ & \left.\frac{\alpha_{p}}{h k \hat{\lambda}_{o}}\left\{\int_{r_{w}}^{r_{f}(t)} q_{i n j}(r, t)\left(\frac{\hat{\lambda}_{o}}{\lambda_{t}(r, t)}-1\right) \frac{d r}{r}\right\}\right]
\end{aligned}
$$

Onde $\frac{\alpha_{p}}{h k \widehat{\lambda}_{o}}\left\{\int_{r_{w}}^{\infty} \frac{\hat{q}_{o}(r, t)}{r} d r\right\}=\Delta \widehat{p_{o}}$, representa a solução monofásica com vazão variável calculada com propriedades da fase óleo @ $S_{w i}$, dada pela equação ( 45 ): 


$$
\Delta \hat{p}_{o}\left(r, t_{N}\right)=\frac{\alpha_{p} B_{w}}{2 h k \hat{\lambda}_{o}} \sum_{j=1}^{n}\left(q_{j}-q_{j-1}\right) \ln \left(\frac{4 \alpha_{t} k \hat{\lambda}_{o}\left(t-t_{j-1}\right)}{e^{\gamma} \hat{c}_{t o} \phi r_{w}{ }^{2}}\right)
$$

Substituindo a equação ( 134 ) na equação ( 133 ):

$$
\begin{array}{r}
p_{w f}-p_{i}=\frac{\alpha_{p} B_{w}}{2 h k \hat{\lambda}_{o}}\left[\sum_{j=1}^{n}\left(q_{j}-q_{j-1}\right) \ln \left(\frac{4 \alpha_{t} k \hat{\lambda}_{o}\left(t-t_{j-1}\right)}{e^{\gamma} \hat{c}_{t o} \phi r_{w}{ }^{2}}\right)\right] \\
+\frac{\alpha_{p}}{h k \hat{\lambda}_{o}}\left[\int_{r_{w}}^{r_{f}(t)} q_{i n j}(r, t)\left(\frac{\hat{\lambda}_{o}}{\lambda_{t}(r, t)}-1\right) \frac{d r}{r}\right]
\end{array}
$$

Sendo assim, para a modelagem do comportamento da pressão em escoamentos bifásicos com vazão variável por meio de soluções analíticas aproximadas adotou-se a seguinte metodologia:

- Cálculo da a solução monofásica com vazão variável calculada com propriedades da fase óleo @ $S_{w i}$, dada pela equação ( 45 );

- Cálculo da posição da frente de avanço a cada instante, considerando o volume total de água que foi injetado no reservatório até aquele momento, conforme a equação ( 130 );

- Cálculo do perfil de vazões antes da frente de avanço $q_{i n j}(r, t)$, aproximada pela superposição de vazões fornecida pela equação ( 48 );

- Utilização das variáveis calculas na equação analítica aproximada para modelar escoamentos bifásicos com vazões variáveis ( 135 ). 
5

\section{Validação dos Modelos}

Foram desenvolvidos algoritmos para implementar as soluções analíticas apresentadas nesse trabalho (Apêndice $C$ ), utilizando o software Scilab (2018). Cabe destacar que foi utilizada a regra do trapézio como método de integração numérica e o método de Bourdet para cálculo da derivada (Apêndice B)

\section{1.}

\section{Comparação entre Modelos Analíticos e Modelo Numérico}

Para verificação do modelo do caso de vazão variável apresentado no capítulo 4 desse trabalho, primeiramente foi realizada uma comparação com o resultado apresentado no modelo criado para os períodos de injeção e falloff, dadas pelas equações ( 91 ) e ( 106 ), respectivamente.

Para isso, a formulação apresentada no capítulo 4 foi utilizada considerando-se dois períodos, de injeção e falloff e comparou-se as derivadas logarítmicas das soluções.

Em seguida, para validação dessas soluções, acrescentou-se uma comparação com a derivada logarítmica de uma solução numérica gerada pelo software IMEX (2017), utilizando-se os mesmos dados de rocha e fluido e o mesmo esquema de vazão.

Essas comparações foram realizadas para dois casos diferentes, o primeiro considerando a razão de mobilidade, $M$, maior do que 1 , e o segundo considerando $\mathrm{M}$ menor do que um.

Os gráficos da Figura 10 e da Figura 11 apresentam o comportamento da pressão ao longo do tempo para os dois casos. 




Ũ

Figura 10 - Comportamento da pressão no modelo onde $\mathbf{M}>1$

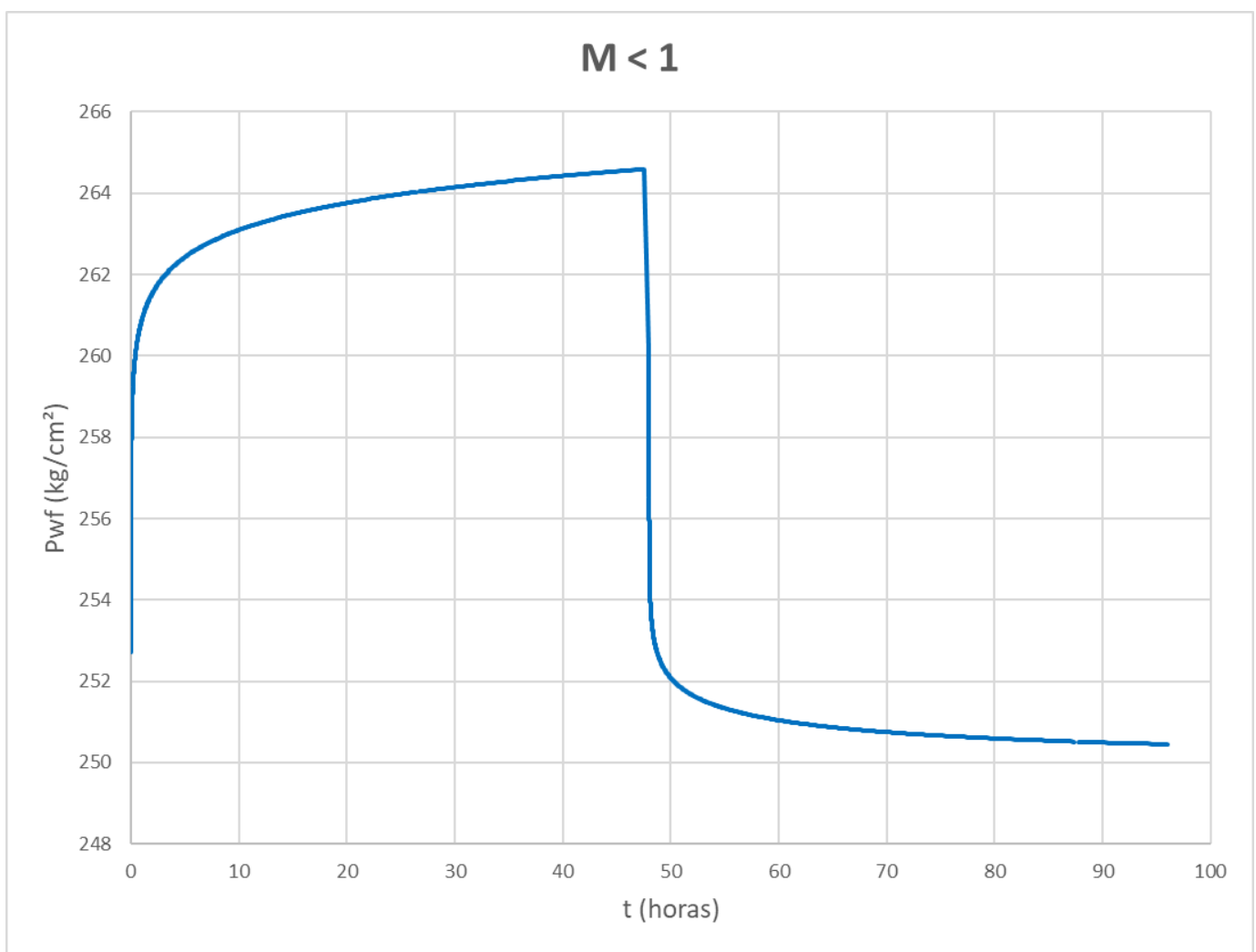

Figura 11 - Comportamento da pressão no modelo onde $\mathrm{M}<1$ 
As comparações entre os gráficos das derivadas logarítimicas de Bourdet (Apêndice B) dos três modelos, para os períodos de injeção e falloff, nos dois casos, são apresentadas nos gráficos a seguir.



Figura 13 - Comparação no período de falloff para $\mathrm{M}>1$ 


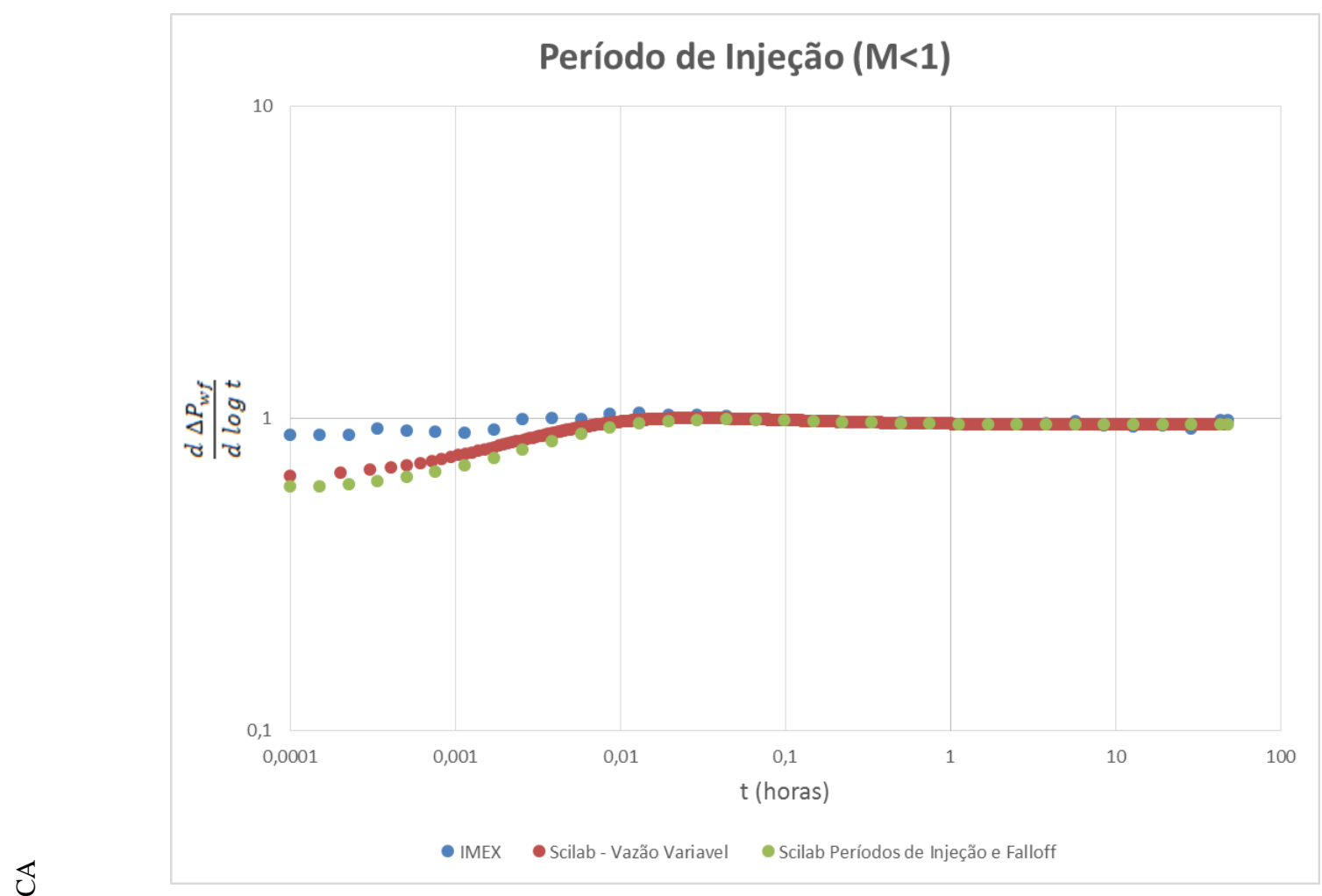

Figura 14 - Comparação no período de injeção para $\mathrm{M}<1$

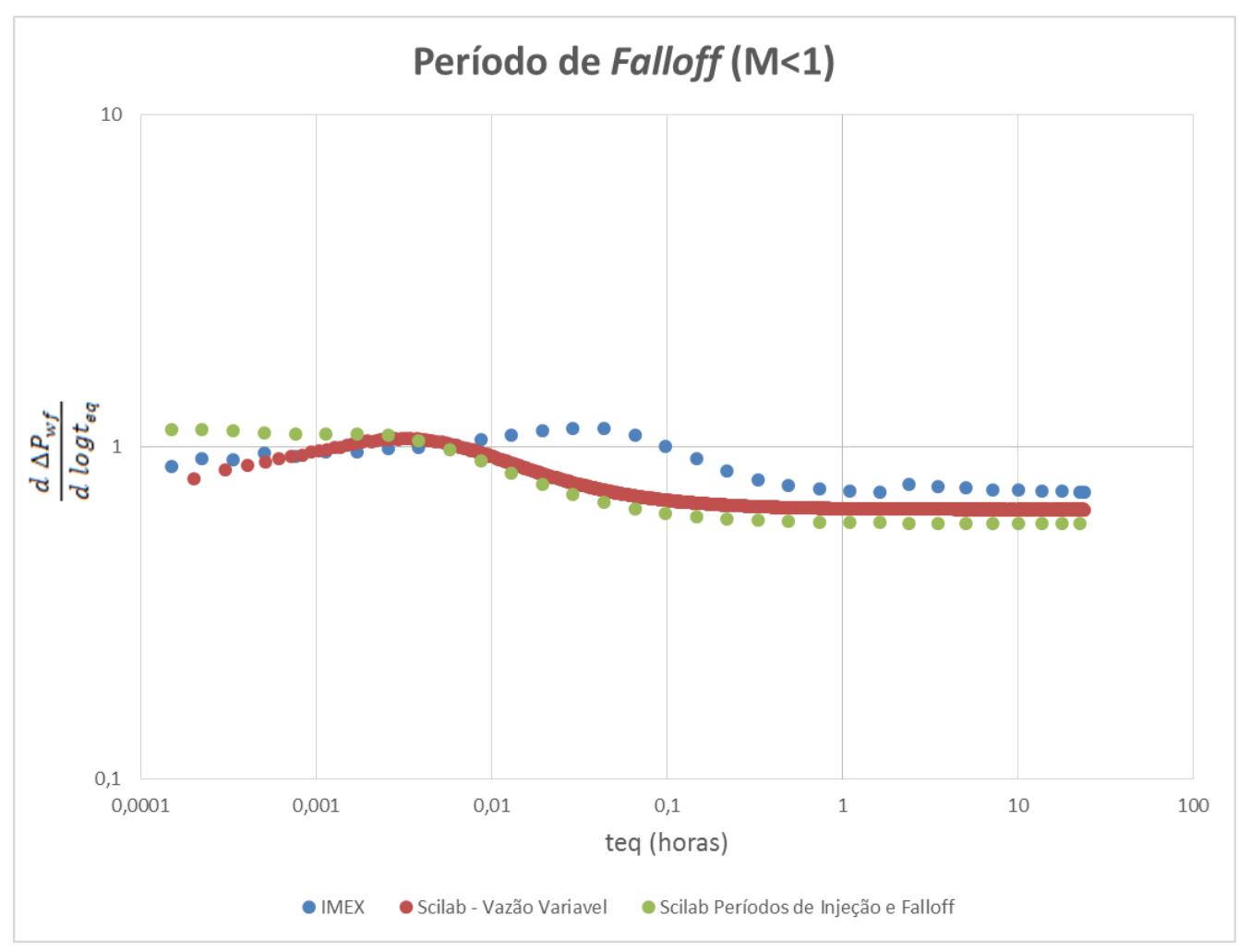

Figura 15 - Comparação no período de falloff para $\mathrm{M}<1$ 
É possível observar que para ambos os casos, as curvas dos três modelos possuem a mesma tendência.

\section{2.}

Comparação entre Modelos de Vazão Variável para Escoamento Bifásico e para Escoamento Monofásico.

Analisaremos agora o comportamento das curvas geradas pelo modelo de vazão variável, em comparação com as curvas geradas por um modelo de escoamento monofásico, a fim de examinar o comportamento da pressão.

Considerou-se ainda um período de injeção seguido por um período de falloff.

A Figura 16 faz uma comparação do comportamento da pressão entre um teste de produção e um de injeção, para a razão de mobilidade maior do que 1 enquanto a Figura 17 faz essa mesma comparação para a razão de mobilidade menor que 1.

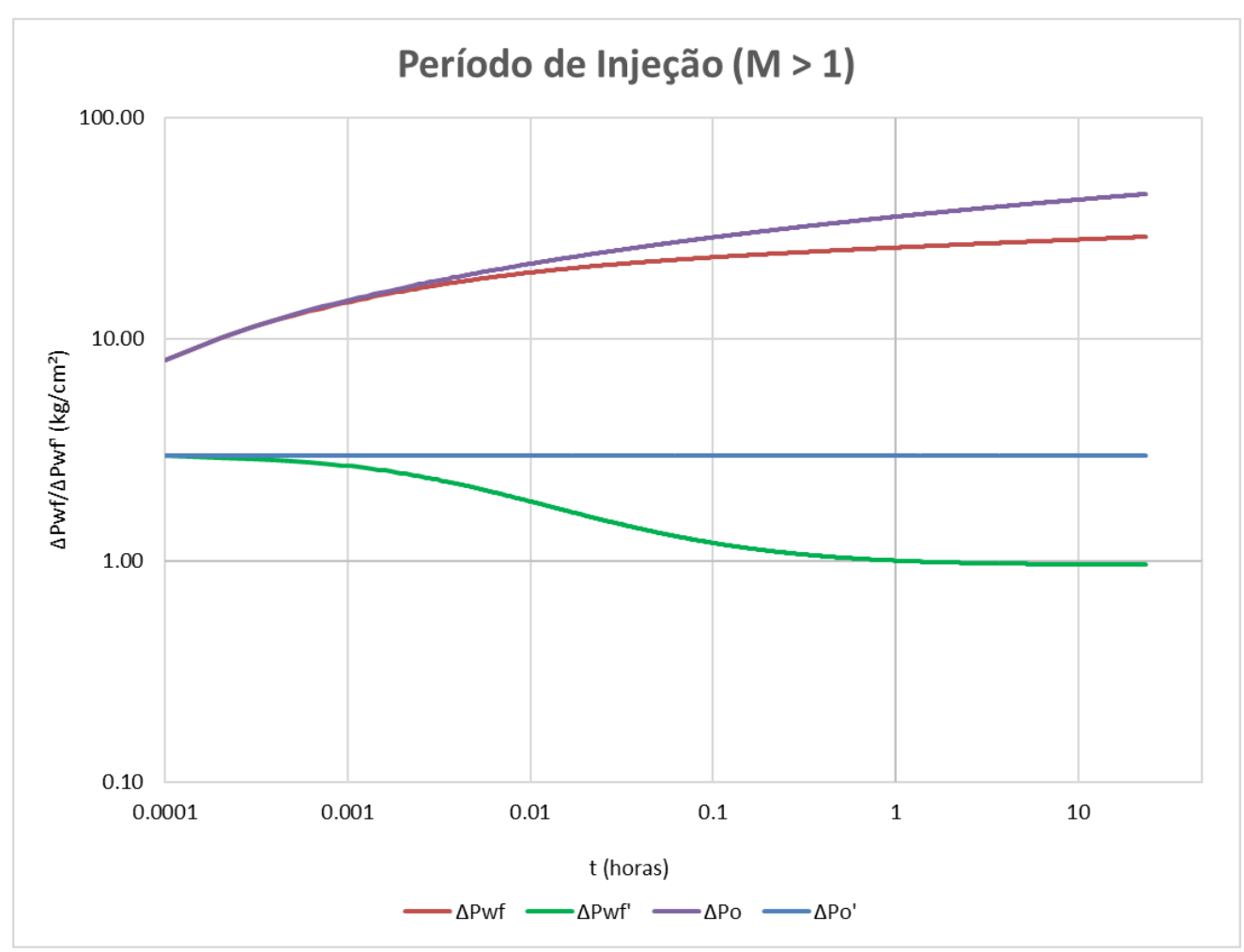

Figura 16 - Período de fluxo monofásico X Período de injeção - M > 1 


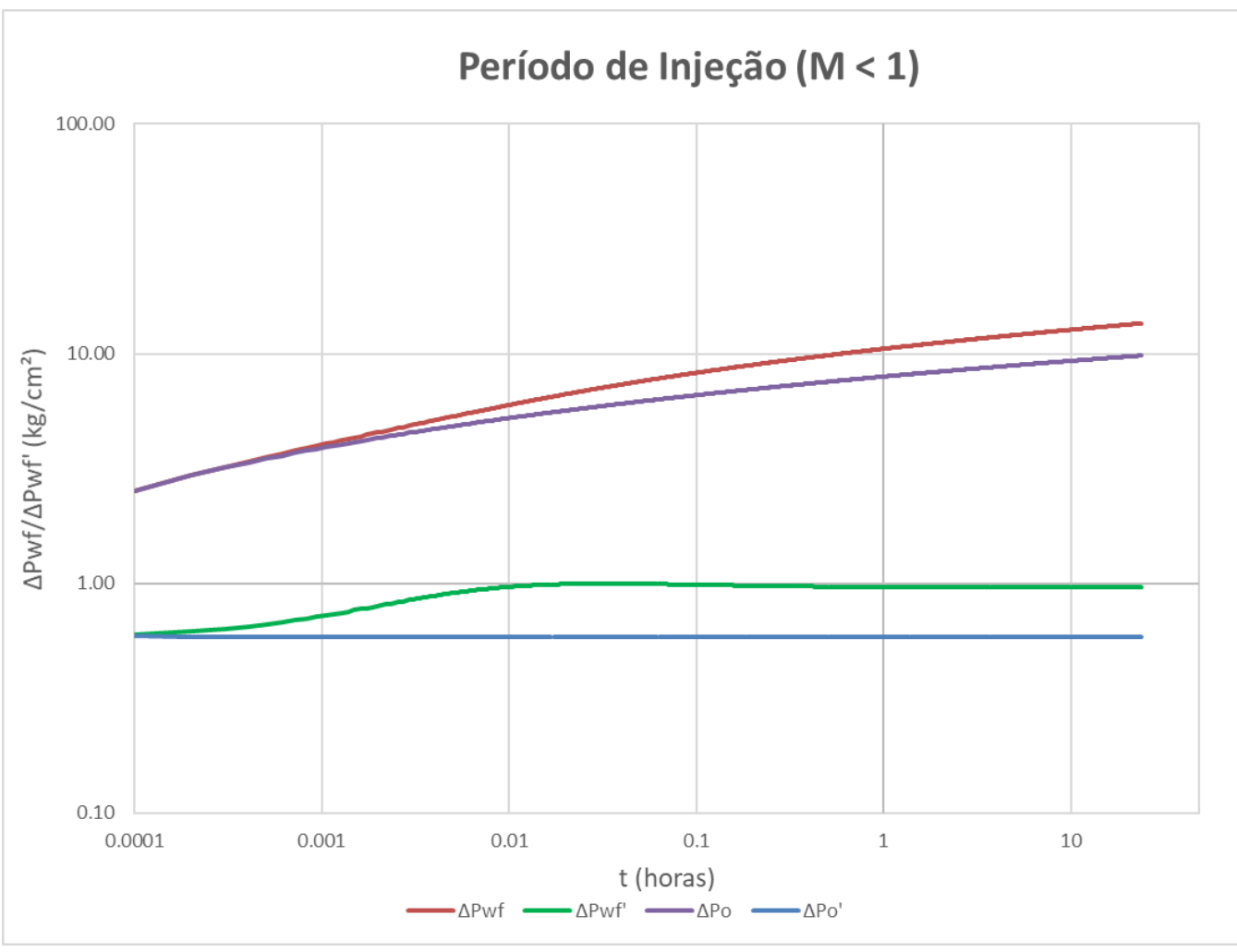

Figura 17 - Período de fluxo monofásico X Período de injeção - M $<1$

Observa-se que, inicialmente, a derivada da pressão de fundo de poço medida durante o período de injeção se aproxima da derivada da pressão de fundo durante o escoamento monofásico. Esse comportamento era esperado, uma vez que inicialmente o reservatório está saturado com óleo. 


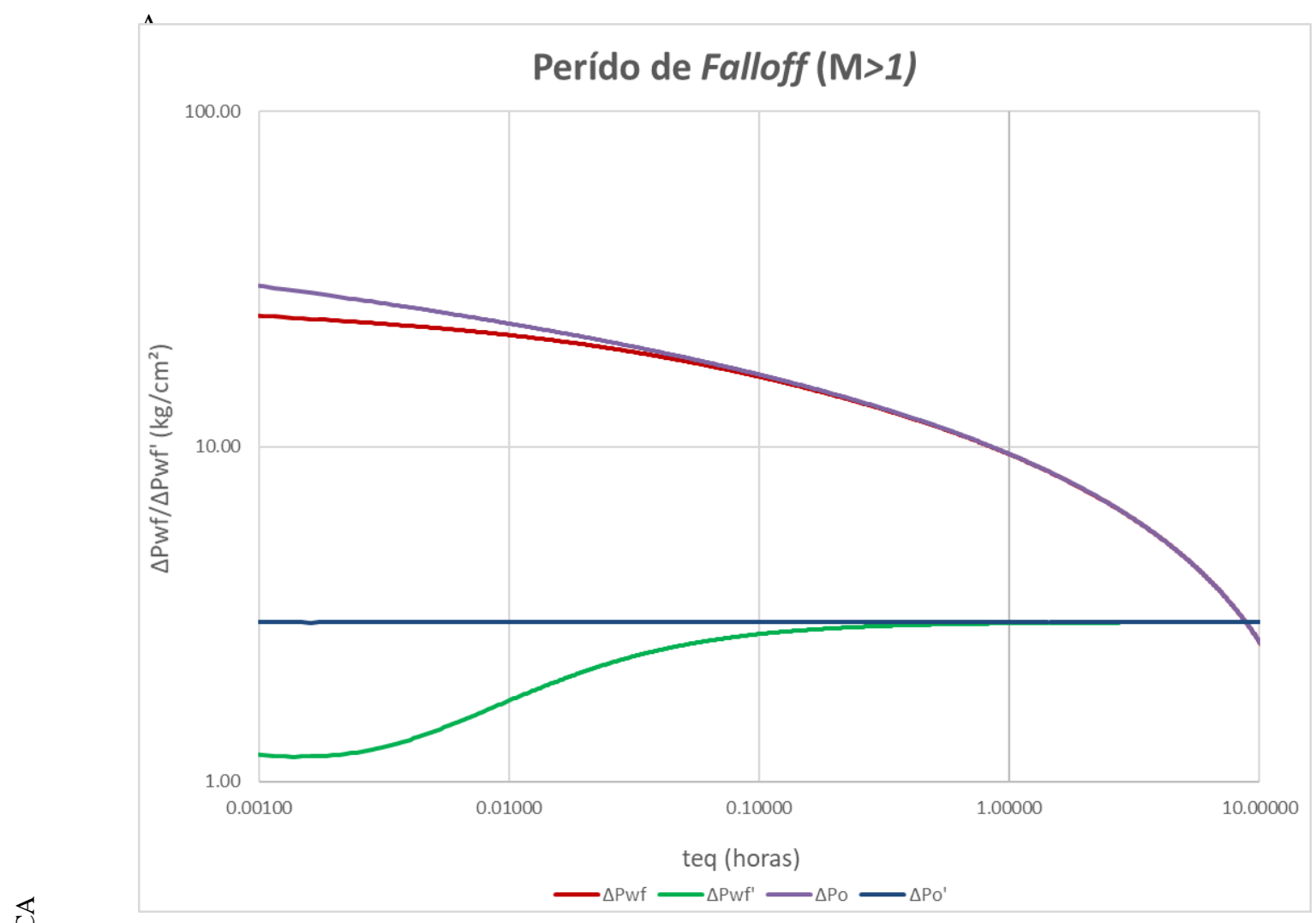

Figura 18 e Figura 19 apresentam uma comparação entre período de fluxo monofásico e o período de falloff:

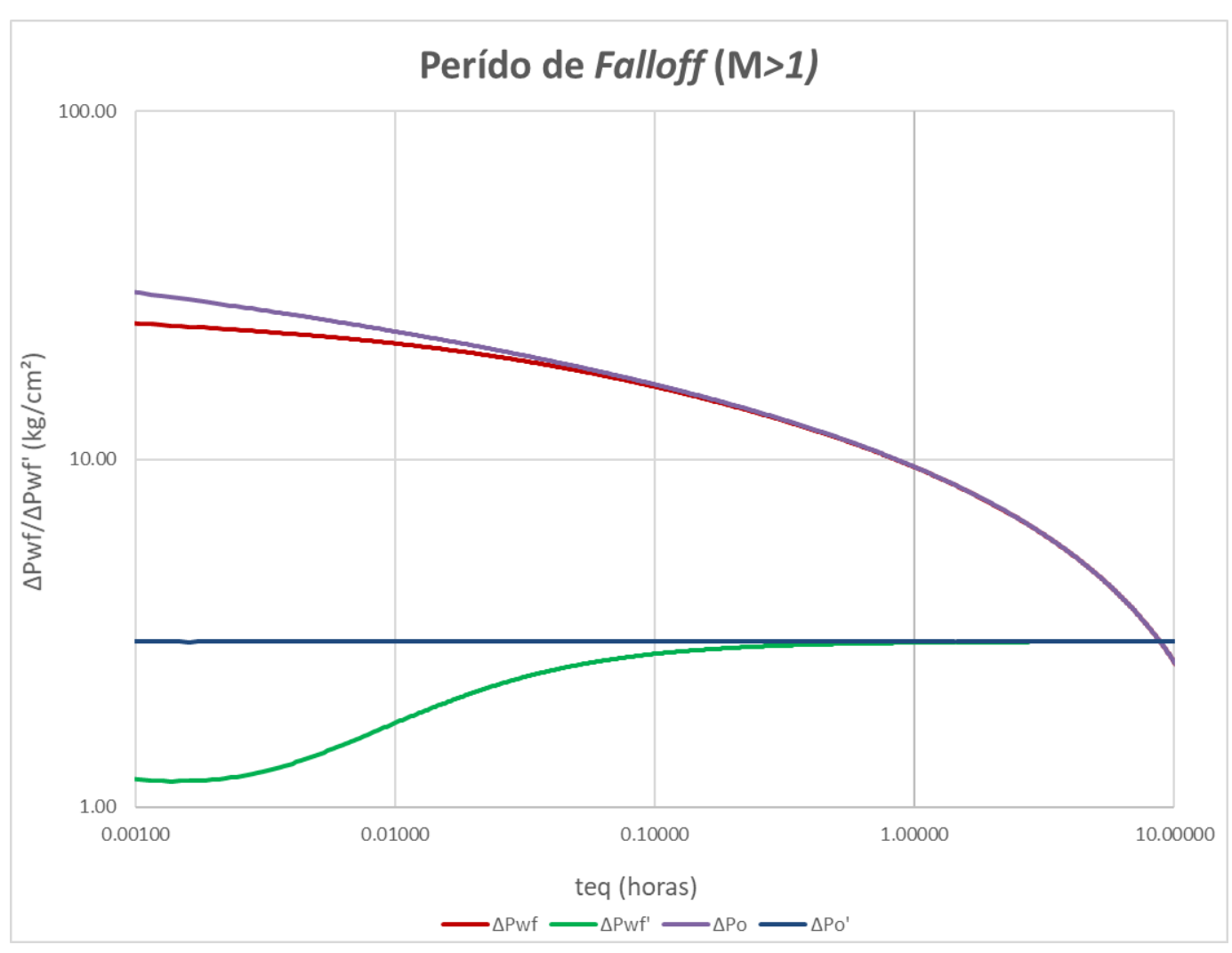

Figura 18 - Período de fluxo monofásico X Período de falloff 


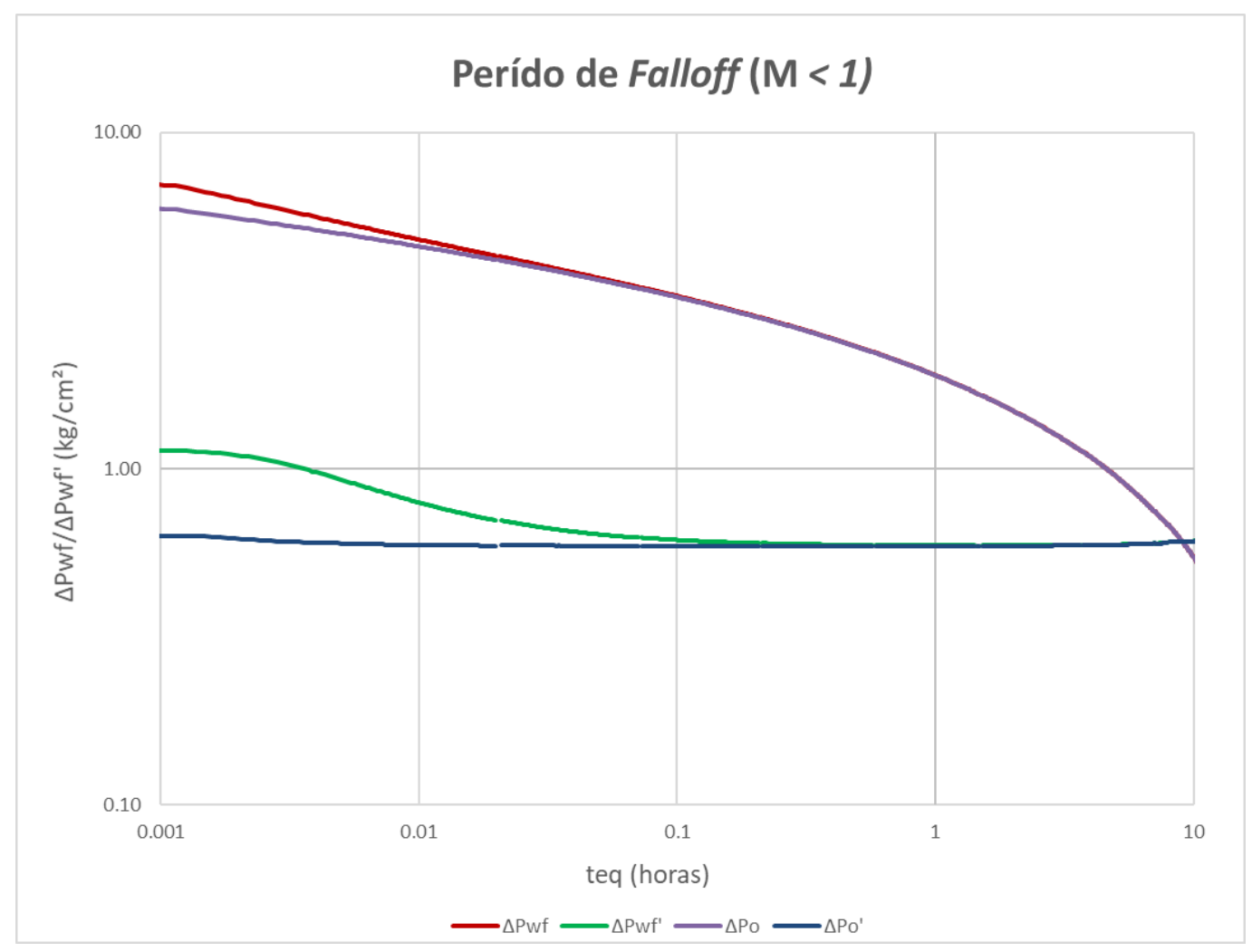

Figura 19 - Período de fluxo monofásico X Período de falloff

Observa-se que, com o passar do tempo, a derivada da pressão de fundo de poço medida durante o período de falloff se aproxima da derivada da pressão de fundo durante o escoamento monofásico. Esse comportamento era esperado, uma vez que, com o avanço da frente de água, o reservatório tende a ficar saturado com água.

\section{3.}

\section{Caso 1 - Modelo com três vazões variáveis}

Com a verificação do comportamento das pressões e suas derivadas, buscou-se ainda a validação do modelo de vazão variável para casos envolvendo outros períodos de vazão.

Primeiramente, foi construído um modelo para o caso de três vazões, de $1000 \mathrm{~m}^{3} / \mathrm{h}, 500 \mathrm{~m}^{3} / \mathrm{h}$ e $100 \mathrm{~m}^{3} / \mathrm{h}$, respectivamente, conforme indicado na Figura 20. 


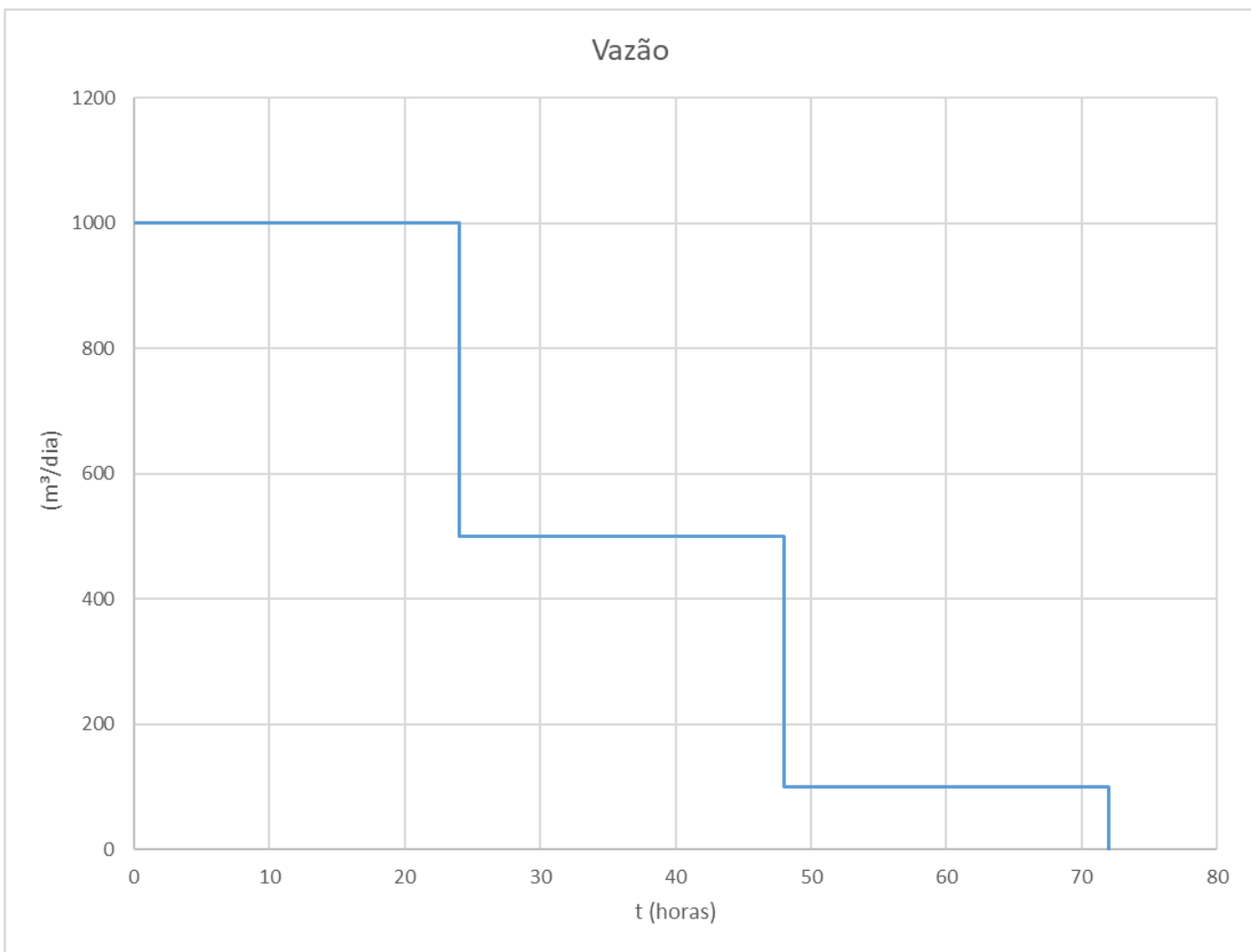

U

Figura 20 - Esquema de vazões do Caso 1.

O comportamento da pressão ao longo do tempo para esse modelo está indicado na Figura 21.

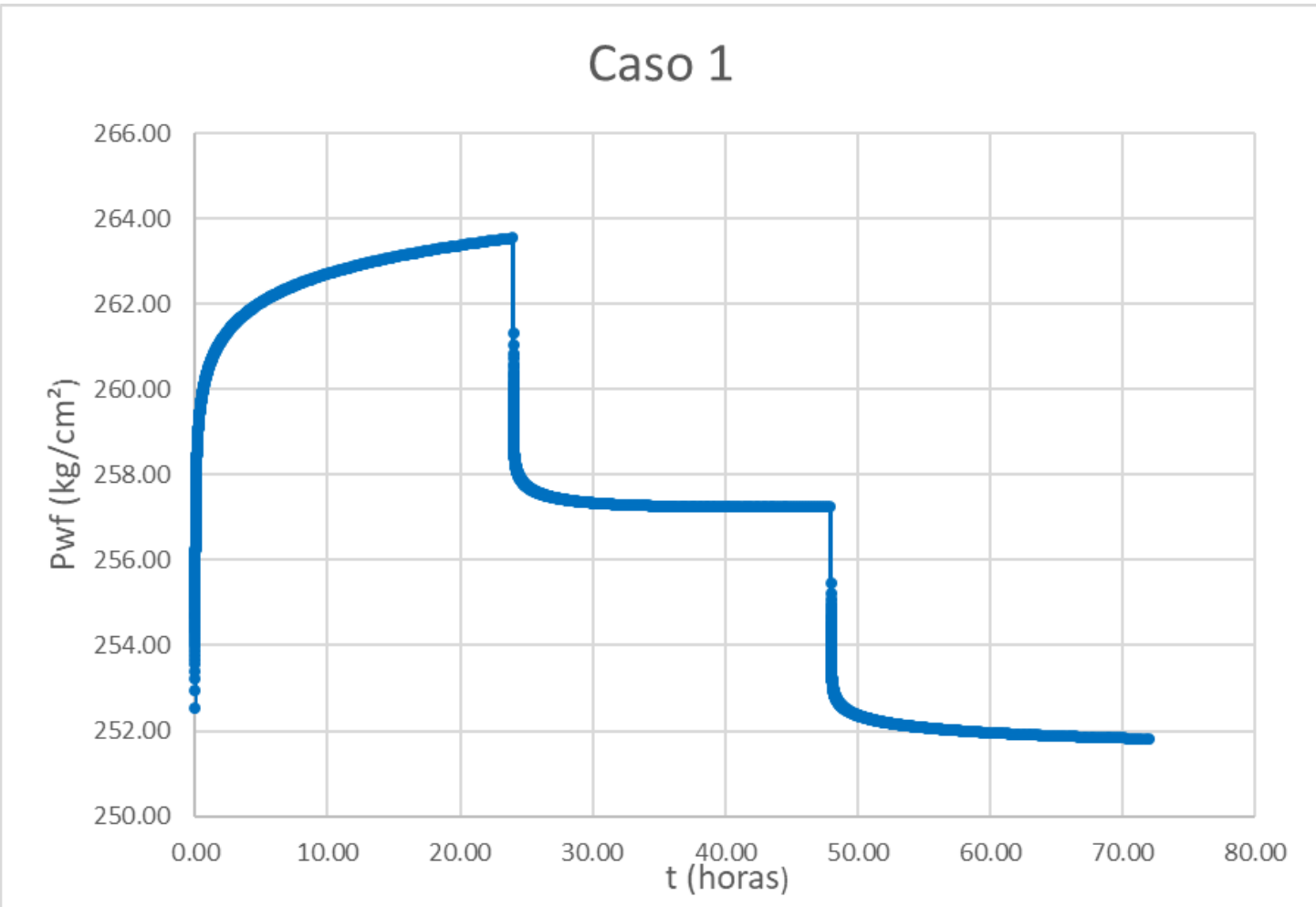




\section{4 .}

\section{Caso 2 - Modelo com quatro vazões variáveis}

Buscando-se ampliar o horizonte abrangido pelo modelo, em seguida foi construído o caso com quatro vazões de injeção variáveis, de $1000 \mathrm{~m} 3 / \mathrm{h}$, $500 \mathrm{~m}^{3} / \mathrm{h}, 200 \mathrm{~m}^{3} / \mathrm{h}$ e $100 \mathrm{~m}^{3} / \mathrm{h}$, respectivamente, conforme indicado na Figura 22, cujo comportamento da pressão é indicado na Figura 23.

Vazão

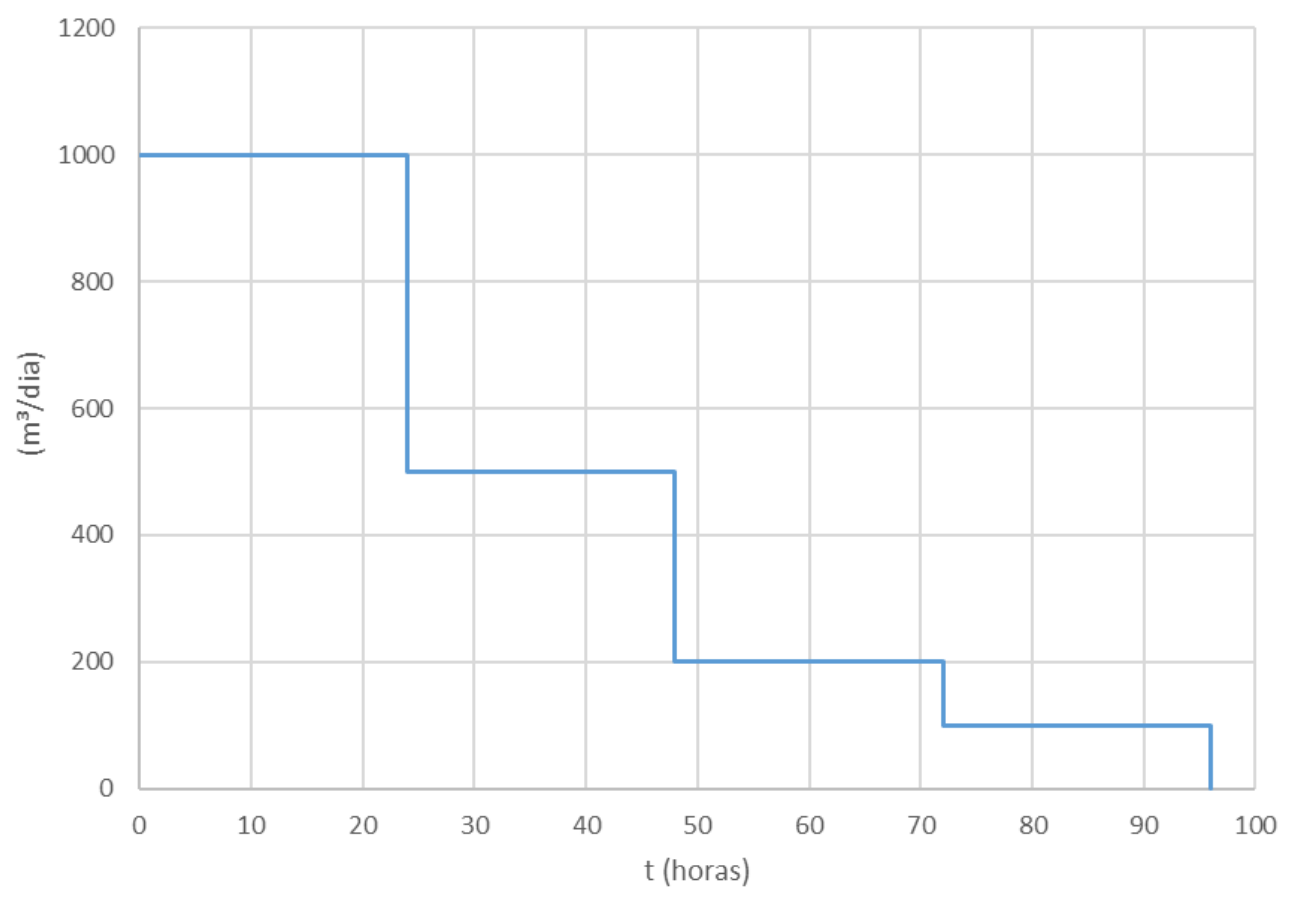

Figura 22 - Esquema de vazões do Caso 2. 


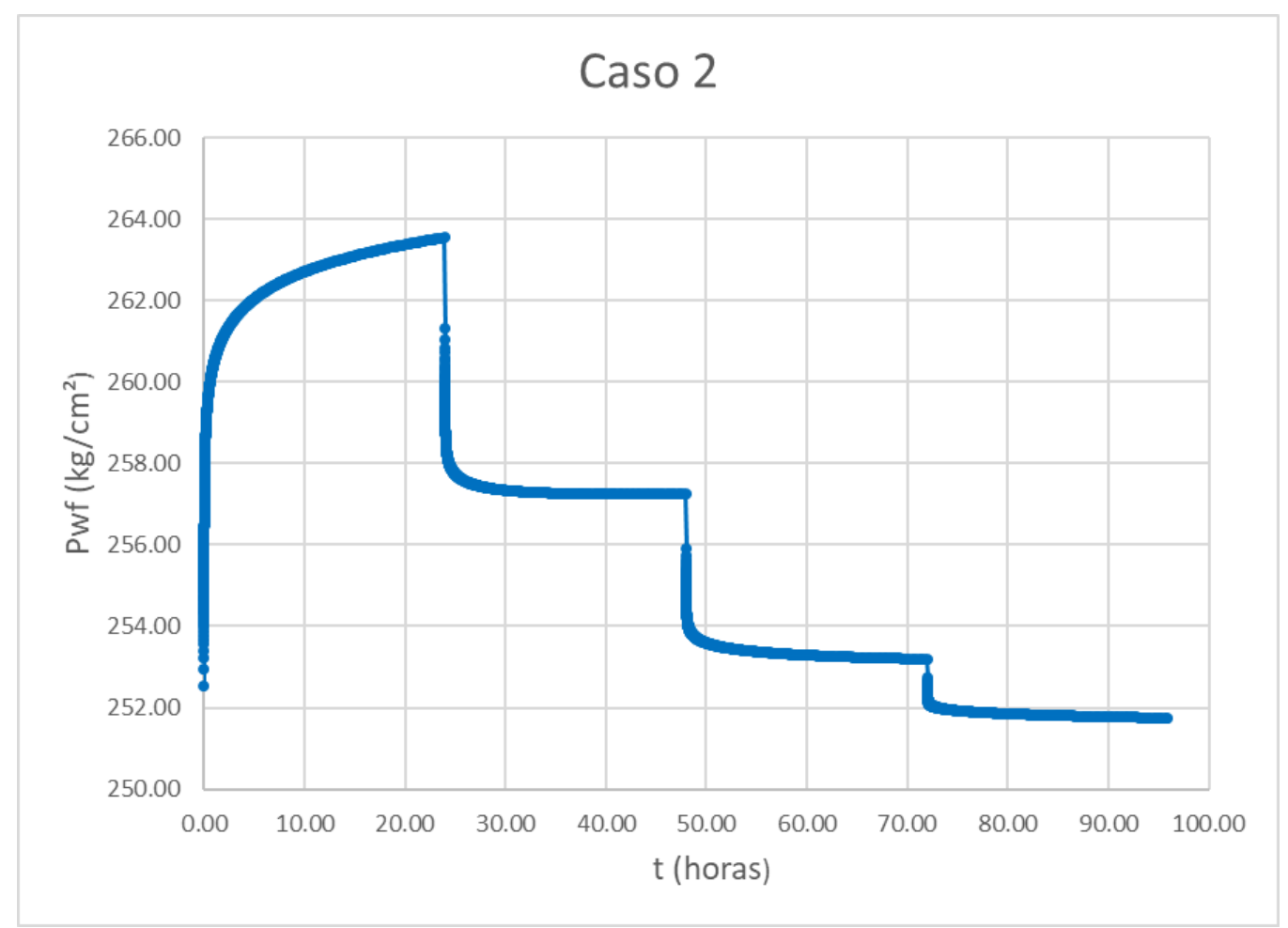

Figura 23 - Caso 2 - Modelo com 4 vazões.

\section{5.}

\section{Caso 3 - Modelo com seis vazões variáveis}

A fim de comparar os resultados para um maior número de vazões, e verificar a eficiência do modelo para testes de injetividade com vazão variável, foram desenvolvidos, no Scilab e no IMEX, modelos com seis vazões variáveis, de $1000 \mathrm{~m}^{3} / \mathrm{h}, 0 \mathrm{~m}^{3} / \mathrm{h}, 500 \mathrm{~m}^{3} / \mathrm{h}, 0 \mathrm{~m}^{3} / \mathrm{h}, 1000 \mathrm{~m}^{3} / \mathrm{h}$ e 0 $\mathrm{m}^{3} / \mathrm{h}$, respectivamente, conforme indicado na Figura 24 


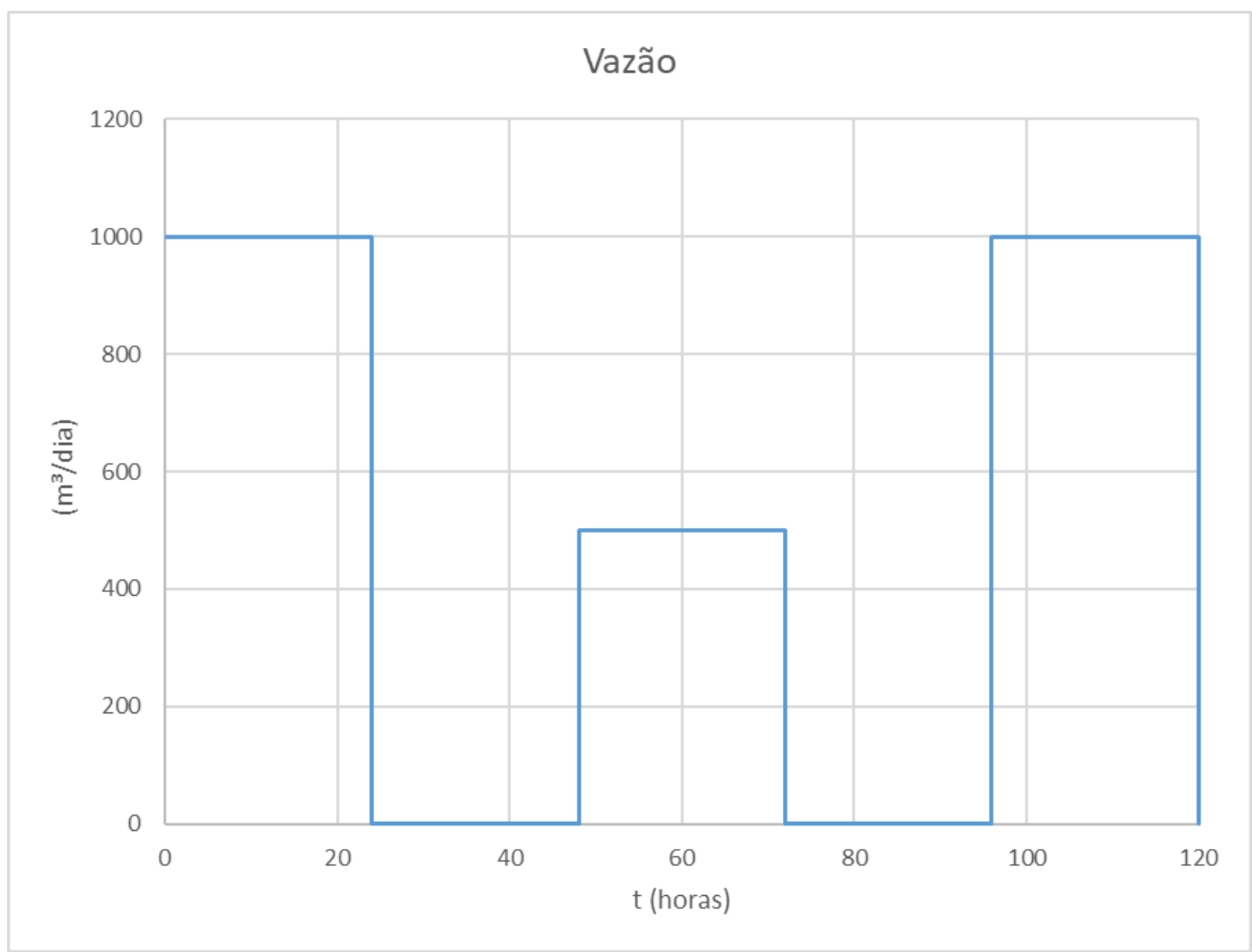

U

Figura 24 - Esquema de vazões do Caso 3.

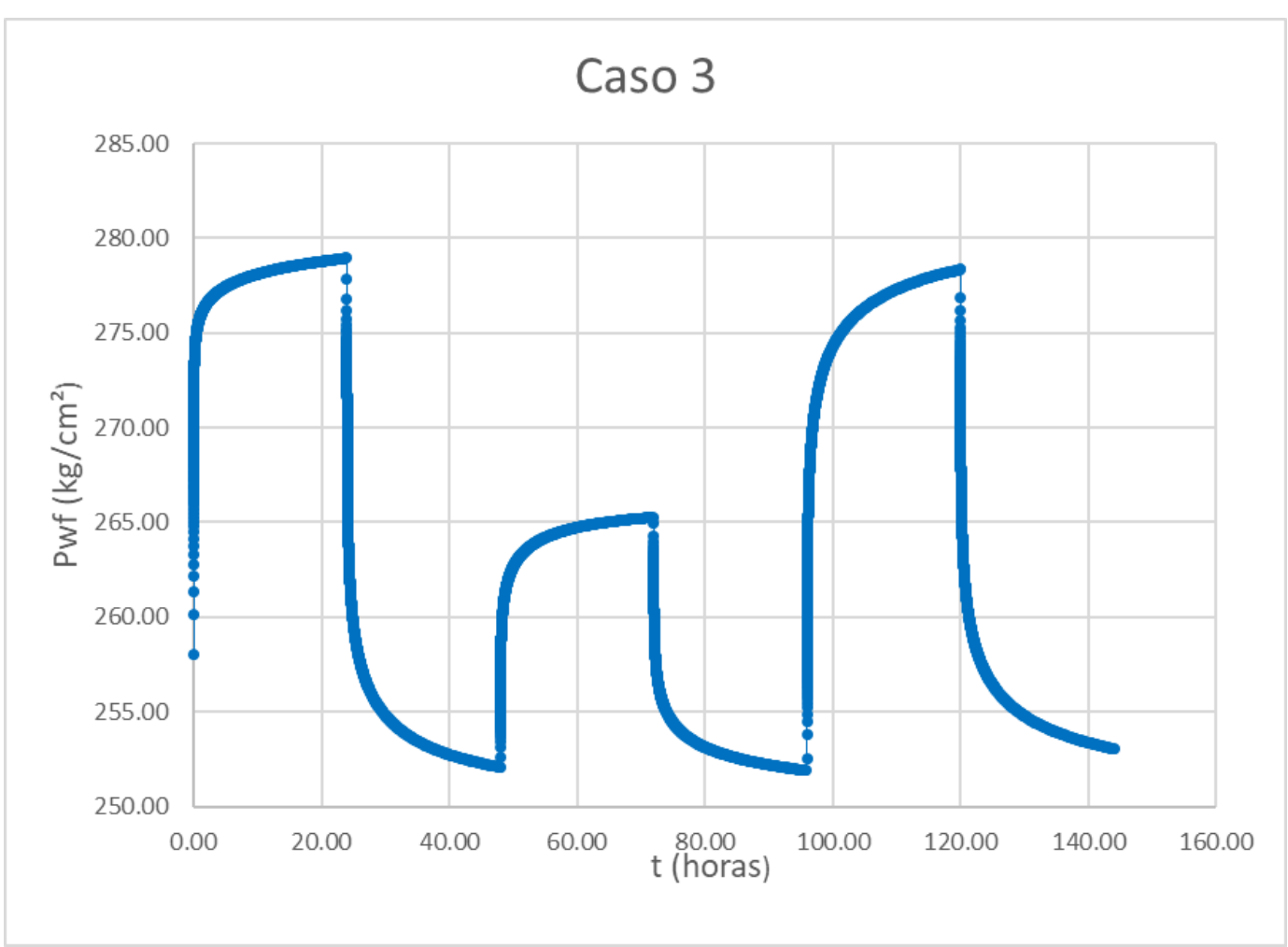

Figura 25 - Caso 3 - Modelo com seis vazões 
Para esse caso, a fim de verificar a eficácia do modelo para casos com um maior número de períodos com diferentes vazões, fez-se também uma comparação das derivadas de cada período.

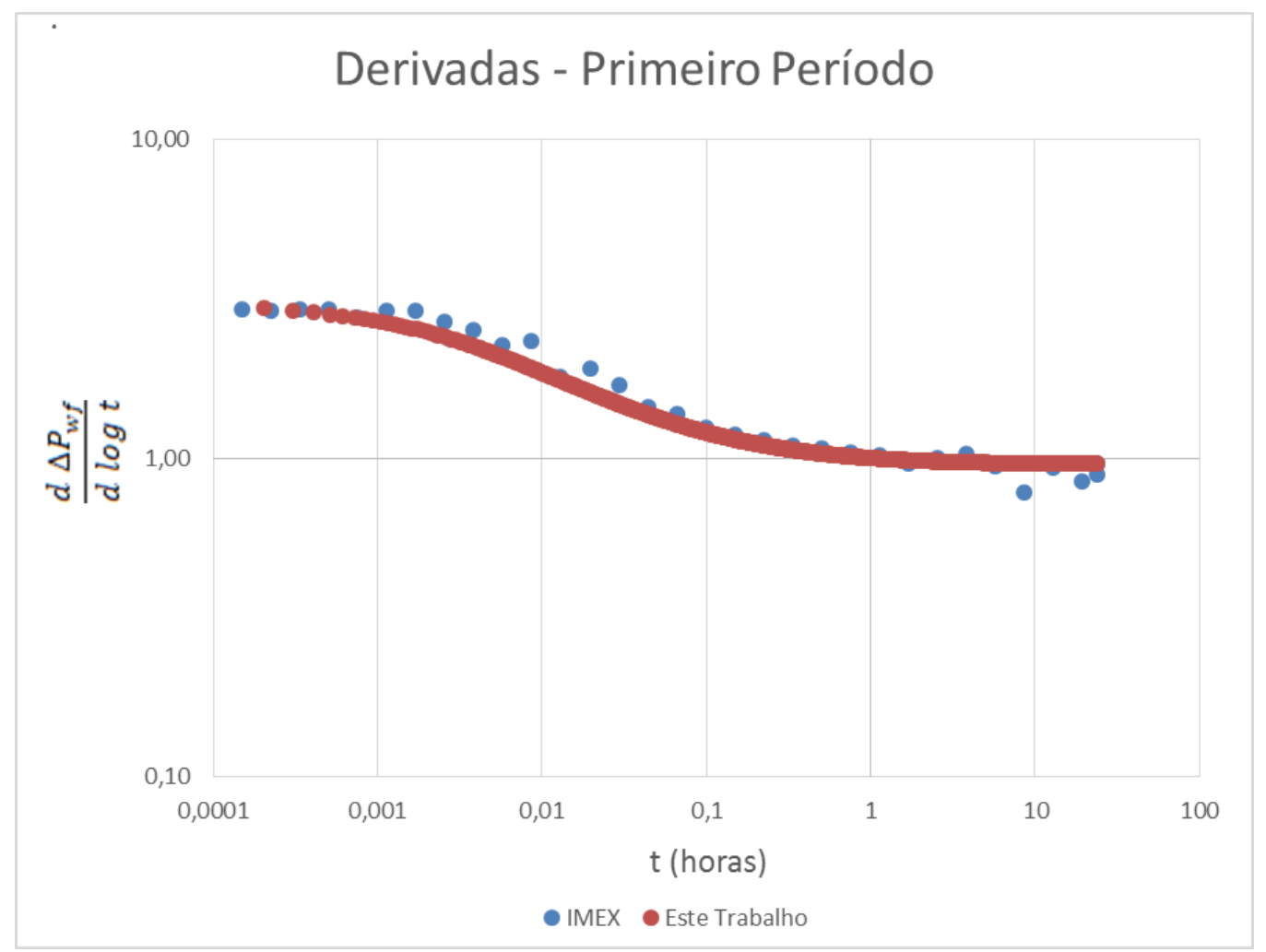

Figura 26 - Comparação entre derivadas - Primeiro período 




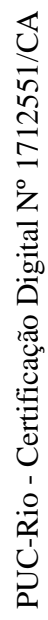

Figura 27 - Comparação entre derivadas - Segundo período

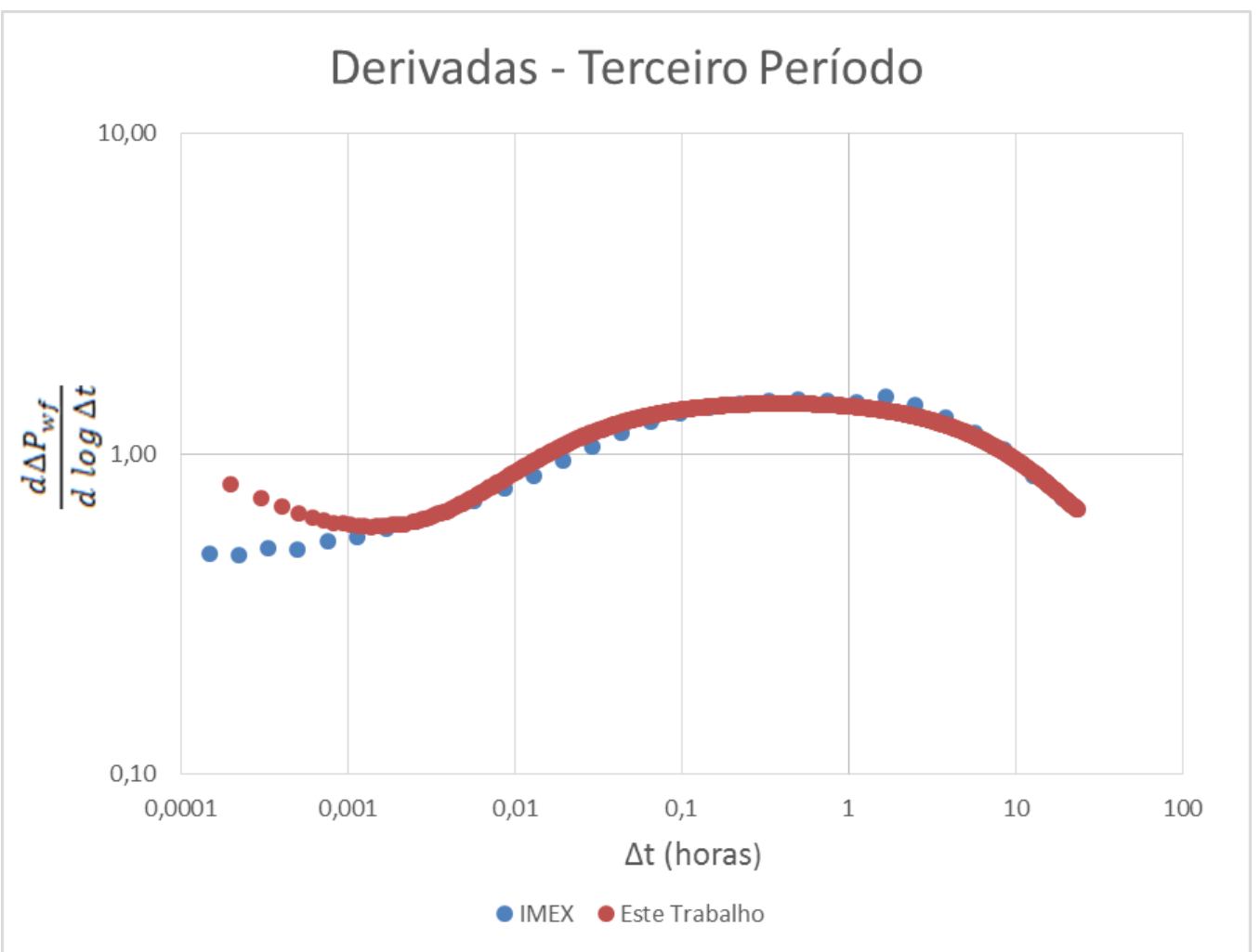

Figura 28 - Comparação entre derivadas - Terceiro período 


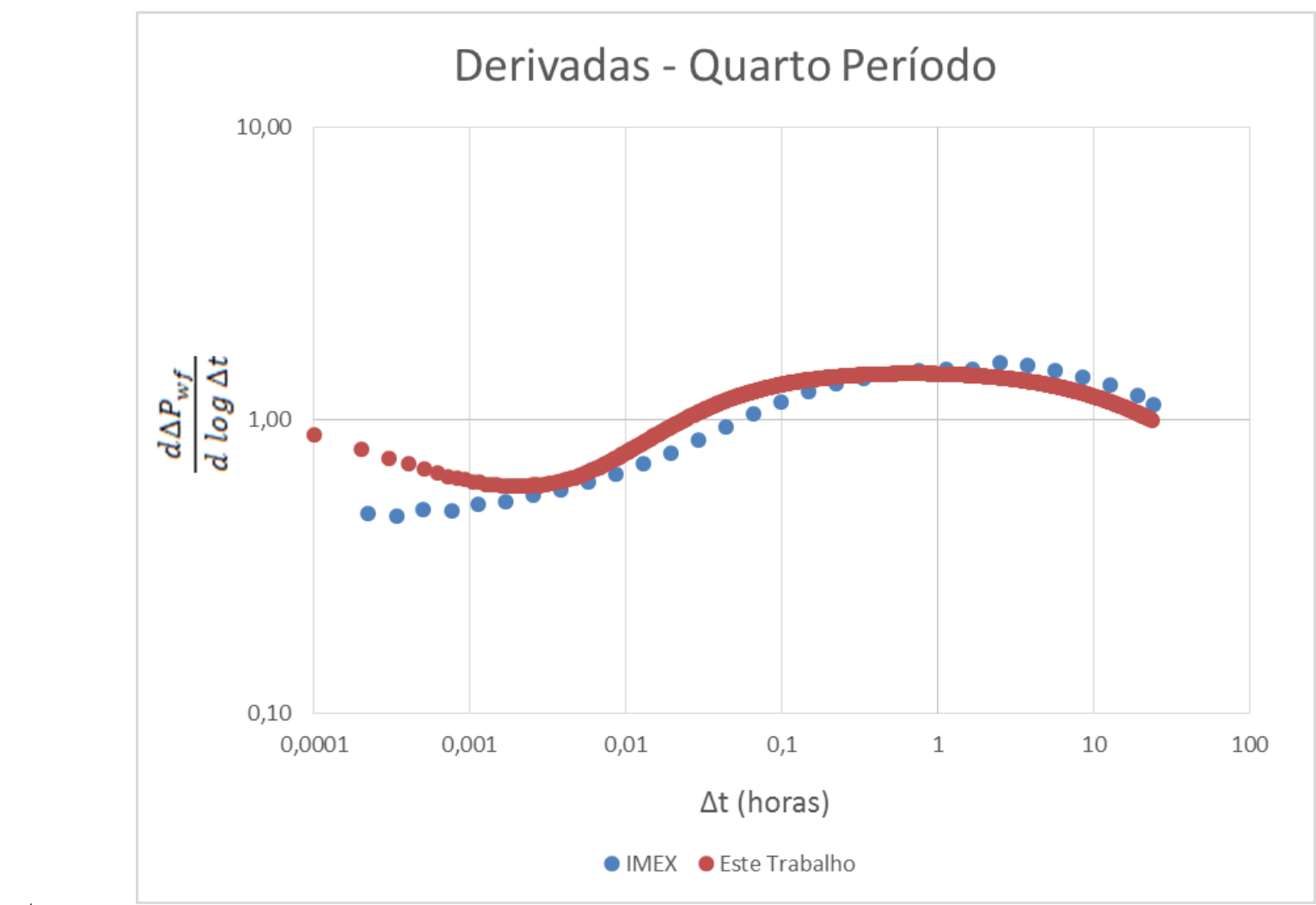

Uू

Figura 29 - Comparação entre derivadas - Quarto período

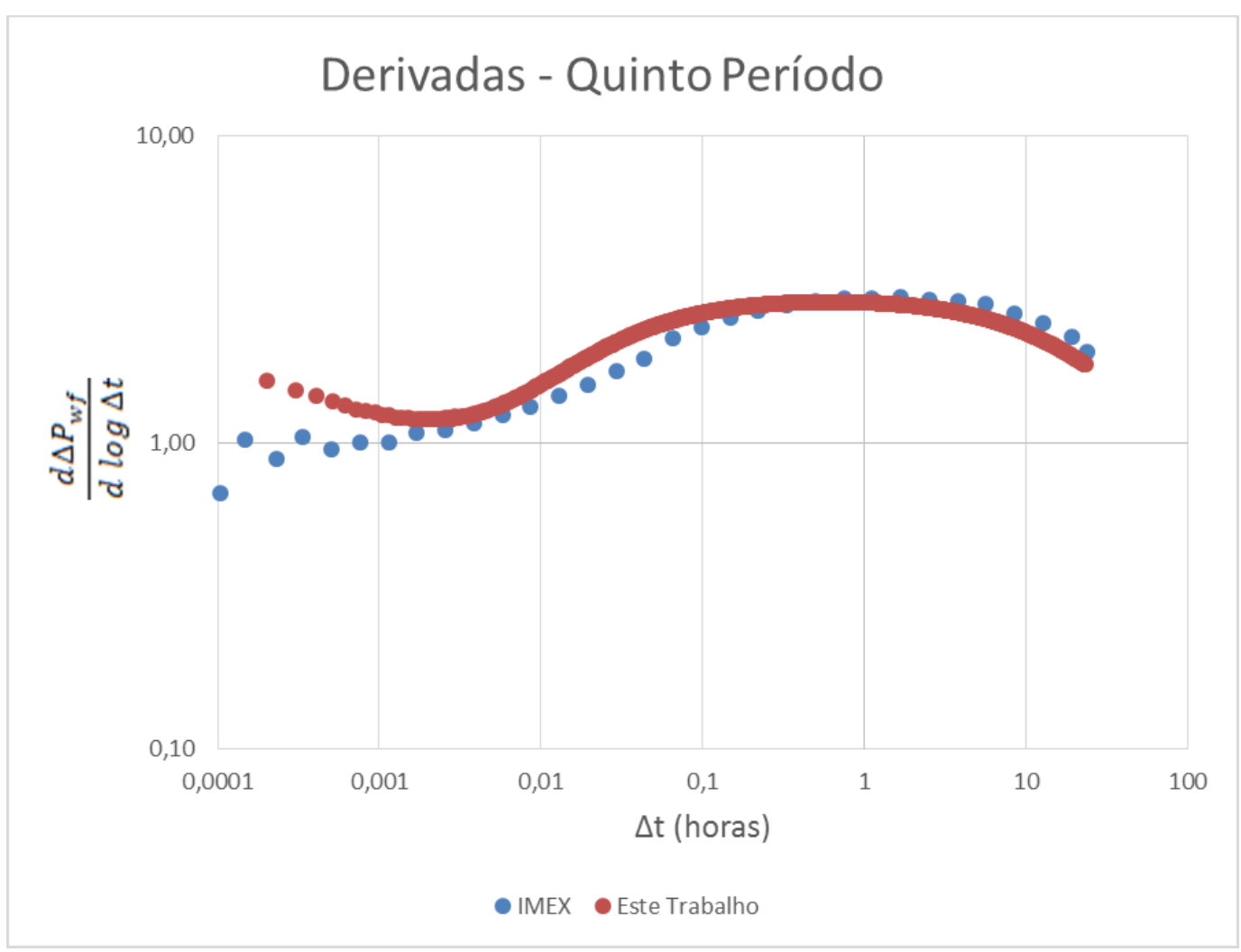

Figura 30 - Comparação entre derivadas - Quinto período 


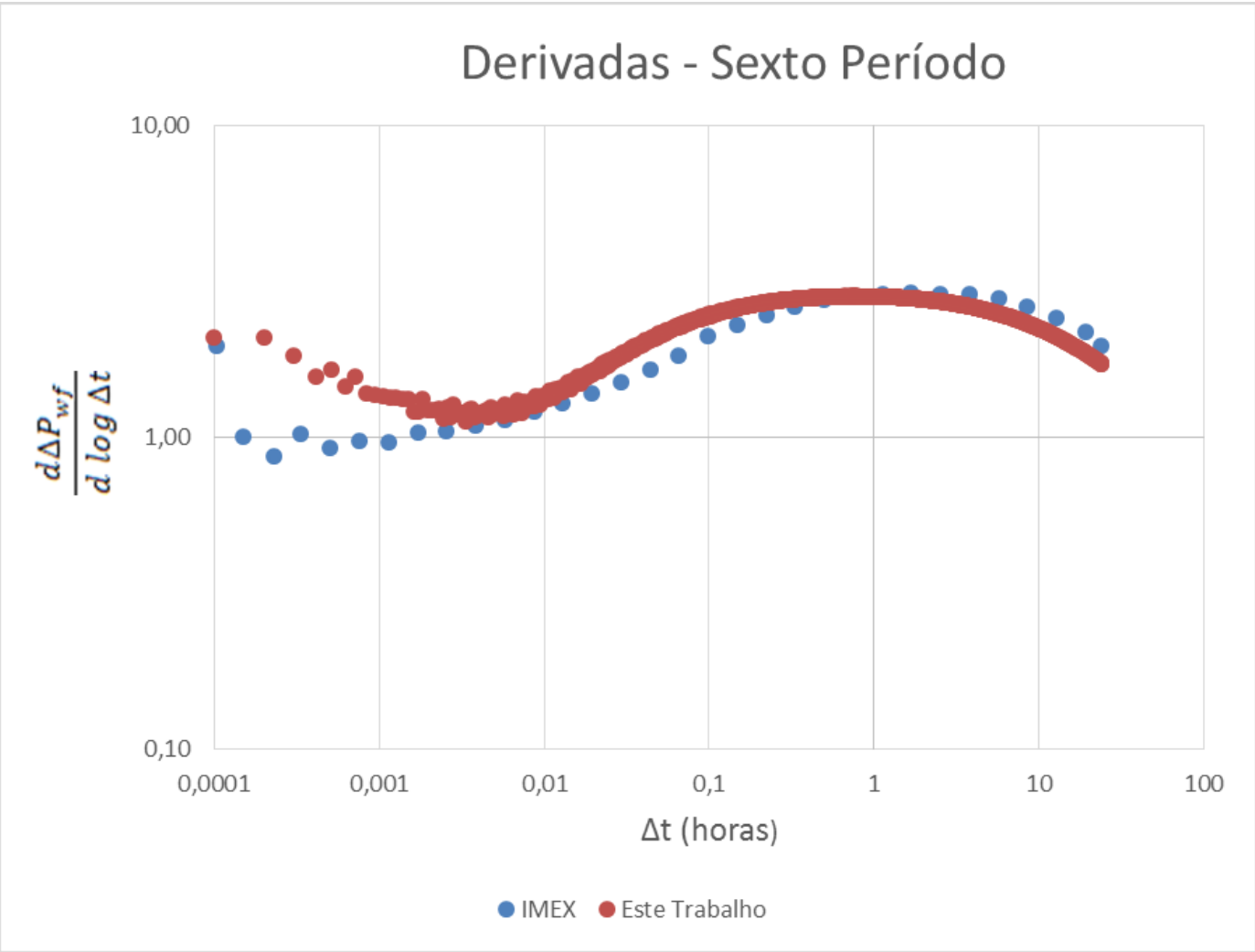

Figura 31 - Comparação entre derivadas - Sexto período

Os gráficos mostram uma boa correlação entre as curvas das derivadas do modelo construído nesse trabalho e as curvas geradas pelo IMEX, o que indica que o trabalho pode ser utilizado para a modelagem de um grande número de períodos com vazões variáveis.

\section{6.}

\section{Cálculo da Permeabilidade Efetiva}

Nessa etapa do trabalho, buscou-se validar o modelo apresentado por meio da comparação da permeabilidade encontrada na curva da resposta da pressão pelo tempo e a permeabilidade inserida como dado de entrada.

Como visto anteriormente, para o caso de vazão constante com escoamento monofásico, a variação na pressão do fundo de poço é dada pela equação ( 40 ): 


$$
\Delta \widehat{p_{o}}(\mathrm{t})=\frac{\alpha_{p} q_{w} B_{w}}{h k \hat{\lambda}_{o}}\left\{\frac{1}{2} \ln \left(\frac{4 \alpha_{t} k \hat{\lambda}_{o} t}{e^{\gamma} \widehat{c_{t o}} \phi r_{w}^{2}}\right)\right\}
$$

Essa equação indica que um gráfico das pressões no poço contra o logaritmo do tempo apresenta uma linha reta, cujo coeficiente angular é dado por:

$$
m=\frac{\alpha_{p} q_{w} B_{w}}{h k \hat{\lambda}_{o}} \frac{1}{2}
$$

Dessa forma, a inclinação da reta permite calcular a permeabilidade, uma vez que essa variável pode ser obtida a partir da mobilidade do fluido.

Já um gráfico semi-log da variação da pressão ao longo do tempo para o primeiro período do caso 3 , fornece inclinações distintas, conforme indicado na Figura 32.



Figura 32 - Variação da pressão de fundo de poço - $1^{\circ}$ período

Como foi visto no subcapítulo 5.2, durante o período de injeção de água em um reservatório de água, inicialmente o comportamento da pressão de fundo de poço se assemelha ao caso de escoamento monofásico do óleo, uma vez que o reservatório está majoritariamente saturado com óleo. De forma que por meio da inclinação inicial, é possível obter a mobilidade do óleo. 


$$
\hat{\lambda}_{o}=\frac{\alpha_{p} q_{w} B_{w}}{m h k} \frac{1}{2}
$$

Com o passar do tempo, o reservatório passa a ser saturado majoritariamente por água, de forma que o comportamento da pressão de fundo de poço se assemelha ao caso de escoamento monofásico da água. Sendo assim, por meio da inclinação final, é possível obter a mobilidade da água.

$$
\hat{\lambda}_{w}=\frac{\alpha_{p} q_{w} B_{w}}{m h k} \frac{1}{2}
$$

O período de falloff pode ser analisado de maneira similar, sendo que nesse período, inicialmente o reservatório está majoritariamente saturado com água e com o tempo volta a ficar saturado com óleo.

Para análise desse período, foi gerado o gráfico da variação da pressão de fundo pelo tempo de Horner. Ressalta-se que o gráfico do tempo de Horner é decrescente, de forma que a inclinação referente ao período inicial está à direita do gráfico.

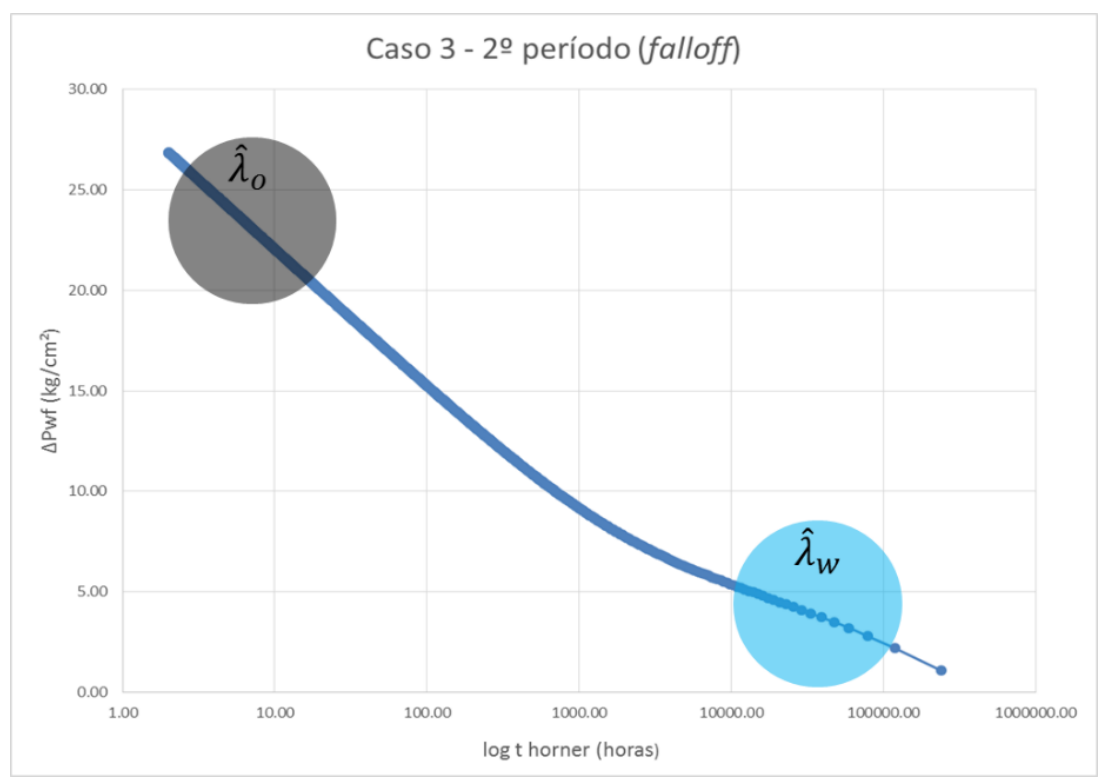

Figura 33 - Variação da pressão de fundo de poço - $2^{\circ}$ período 
Para análise dos demais períodos do Caso 3 , foi gerado o gráfico da variação da pressão de fundo pelo logaritmo da variação do tempo conforme indicado nas figuras seguintes. De forma que, nesse caso, inicialmente o reservatório está saturado majoritariamente por água, e ao final, o reservatório está saturado majoritariamente por óleo, indicando o avanço da frente de avanço de água.

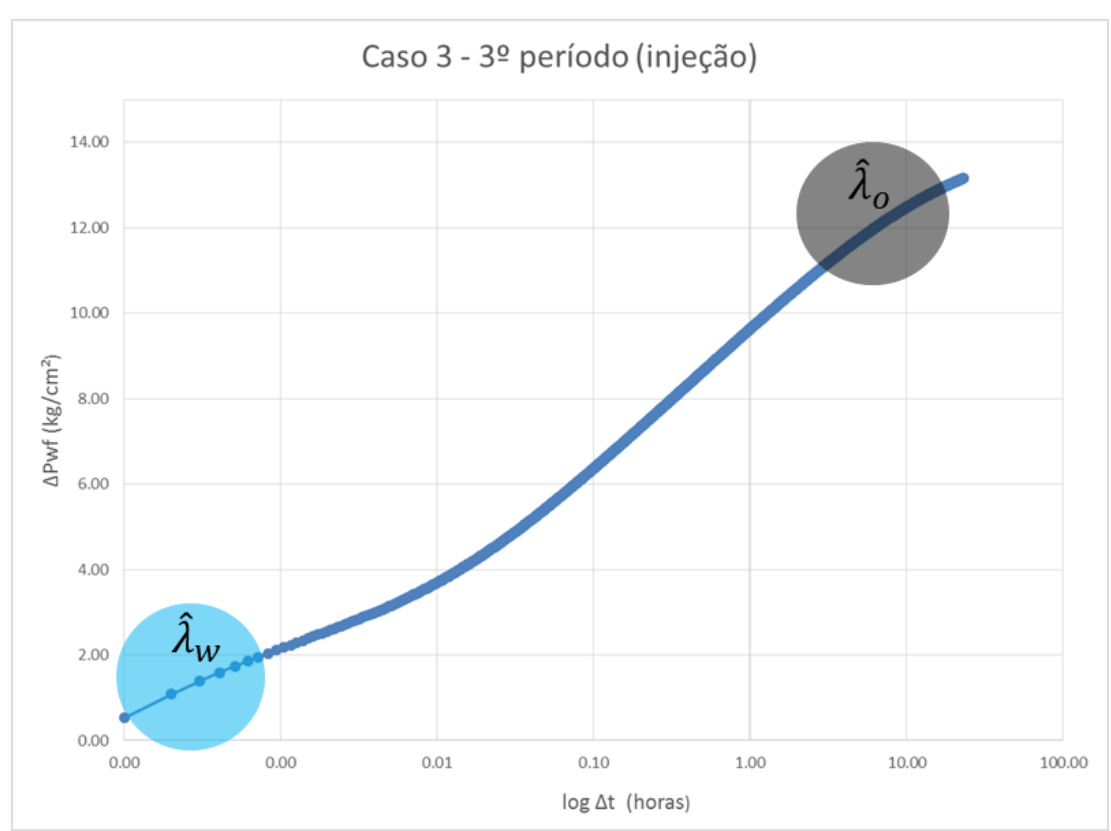

Figura 34 - Variação da pressão de fundo de poço - $3^{\circ}$ período

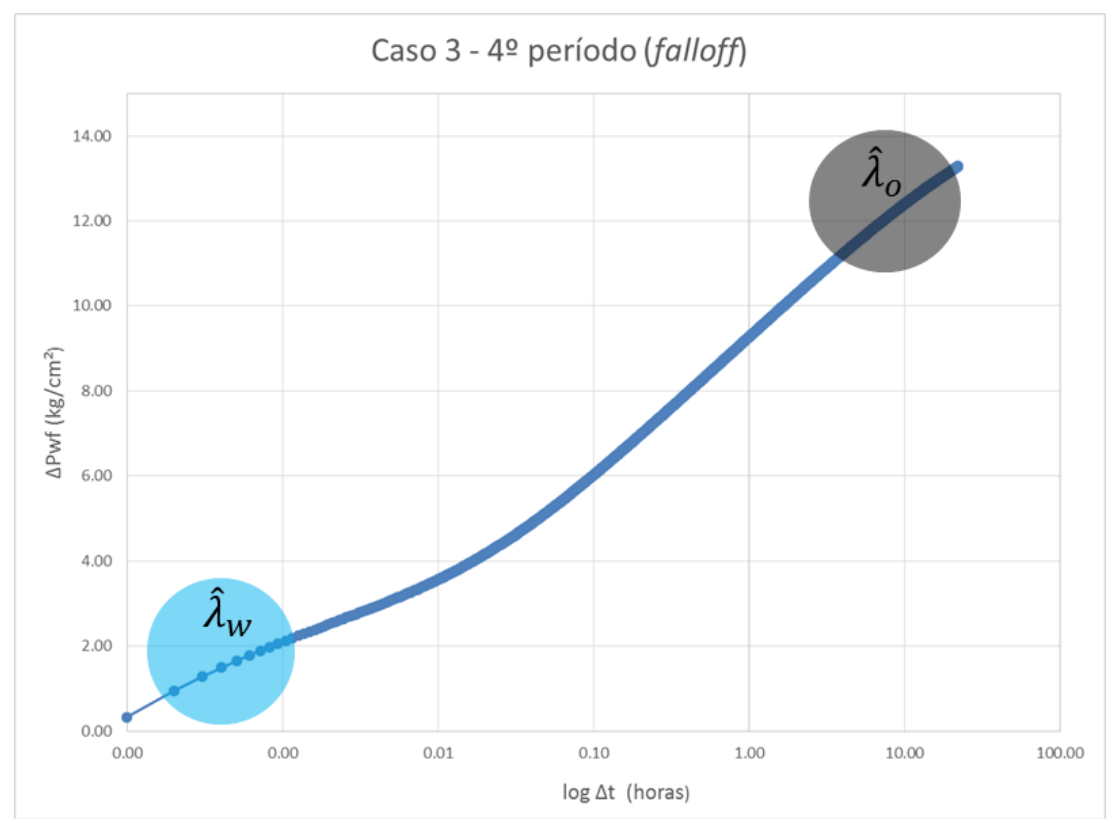

Figura 35 - Variação da pressão de fundo de poço - $4^{\circ}$ período 


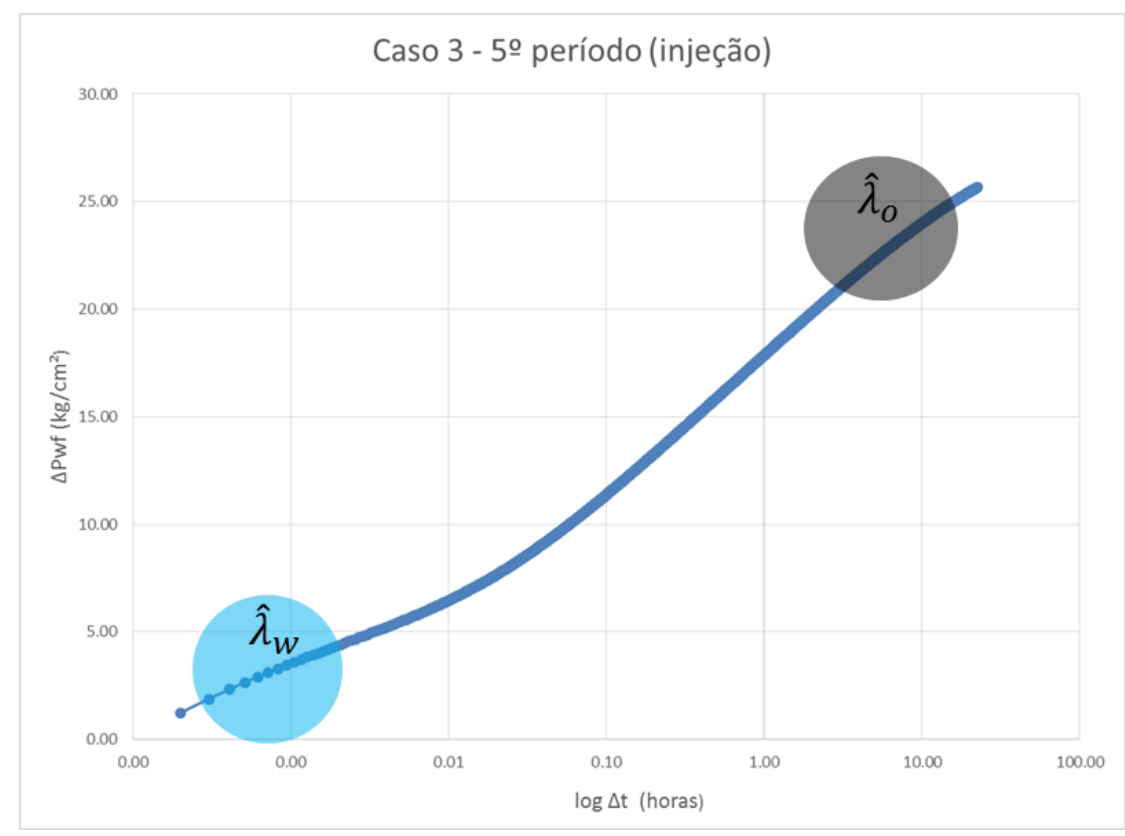

Figura 36 - Variação da pressão de fundo de poço - $5^{\circ}$ período

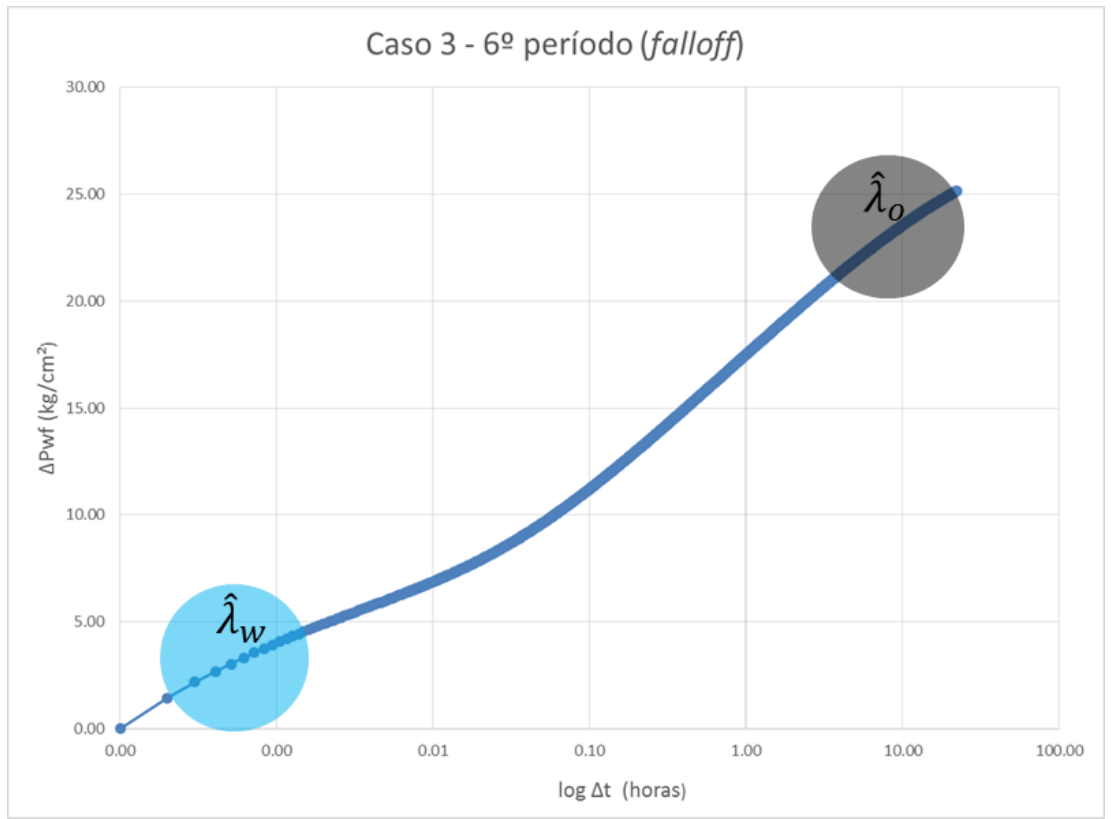

Figura 37 - Variação da pressão de fundo de poço - $6^{\circ}$ período

Com essas considerações, é possível encontrar a permeabilidade por meio de cada período, conforme indicado na seguinte tabela: 
Tabela 2- Cálculo das permeabilidades por período.

\begin{tabular}{|c|c|c|c|c|c|c|}
\hline & 19 período & 20 período & 3ำ período & 40 período & $5 \%$ período & 69 período \\
\hline$q\left(m^{3} /\right.$ dia $)$ & 1000 & 0 & 500 & 0 & 1000 & 0 \\
\hline$m_{0}$ & 6.71 & 6.89 & 3.26 & 3.12 & 6.16 & 6.12 \\
\hline$\hat{\lambda}_{0}$ & 108.76 & 105.92 & 111.82 & 117.07 & 118.60 & 119.35 \\
\hline $\mathrm{K}(\mathrm{mD})$ & 1027.21 & 1000.37 & 1056.12 & 1105.63 & 1142.06 & 1127.21 \\
\hline Erro (\%) & 2.72 & 0.04 & 5.61 & 10.56 & 14.21 & 12.72 \\
\hline$m_{w}$ & 2.21 & 2.76 & 1.36 & 1.37 & 2.99 & 2.77 \\
\hline$\widehat{\lambda}_{w}$ & 330.17 & 264.71 & 268.26 & 265.81 & 244.27 & 263.44 \\
\hline $\mathrm{K}(\mathrm{mD})$ & 992.43 & 795.66 & 806.32 & 798.97 & 734.23 & 791.86 \\
\hline Erro (\%) & -0.76 & -20.43 & -19.37 & -20.10 & -26.58 & -20.81 \\
\hline
\end{tabular}

Os valores de permeabilidade encontrados por meio dos resultados são muito próximos ao utilizado como dado de entrada para a construção do modelo (1000 mD), principalmente quando o reservatório está saturado majoritariamente por óleo.

Sendo assim, é possível estimar a permeabilidade do reservatório a partir de cada intervalo de vazão. 


\section{6}

\section{Conclusão}

Esse trabalho apresentou a construção de uma solução aproximada do comportamento da pressão em testes de injetividade com vazão variável.

A validação dessa solução, realizada por meio da comparação com resultados gerados por um software comercial para um mesmo modelo, indicou que o comportamento da pressão de fundo de poço pode ser obtido por meio dessa solução, de forma que é possível avaliar o reservatório durante e após a conclusão desses testes por meio do modelo construído.

Além disso, esse trabalho indica que é possível estimar a permeabilidade do reservatório a partir de qualquer período de fluxo ou estática. 


\section{Referências Bibliográficas}

ABBASXADEH, M.; KAMAL, M. Pressure-Transiente Testing of Water Injection Wells. SPE- 16744-PA, Sepere 4(1): 115-124.1989.

BARRETO Jr, A.; PERES, A.; PIRES, A. Water Injectivity Tests on Multilaayered Oil Reservoirs. SPE International, SPE Paper 142746, Macaé, junho de 2011.

BRATVOLT, R. B.; HORNE, R. N. Analysis od Pressure-Falloff Tests Following Cold-Water Injection. SPE 18111 - PA. SPEFE 5(3): 293-302. 1990.

BOURDET, D. Well Test Analysis: The Use of Advanced Interpretatiom Models. Handbook of Petroleum Exploration and Production, 3. Series Editor John Cubitt. Elsevier Science B.V. 2002. Paris, France.

BUCKLEY, S. E.; LEVERETT, M. C. Mechanism of Fluid Displacement in Sands. Transactions AIME, 146, p. 107-116, 1942.

GRINGARTEN, A.C. Analysis of an Extended Well Test To Identify Connectivity Between Adjacent Compartments in a North Sea Reservoir. SPE Europec/ EAGE Annual Conference, 2005.

OLIVEIRA, C. M; CORDEIRO, D. C.; TREVIZANI, A. A.; CANZIAN, E. P.; ASSUNÇÃO, G. G. ROMERO, O. J. Análise paramétrica do deslocamento de óleo em um meio poroso governado pela teoria de Buckley-Leverett. Latin American Journal of Energy Research, 2014. 
PERES, A. M. M.; BOUGHRARA, A.A; CHEN, S.; MACHADO, A. A. V; REYNOLDS, A. C. Approximate Analytical Solutions the Pressure Response at a Water Injection Well. SPE International, SPE Paper 90079, Houston, setembro de 2004.

PERES, A. M. M.; BOUGHRARA, A.A; REYNOLDS, A. C. Rate Superposition for Generating Pressure Falloff Solutions. SPE Journal, SPE Paper 84957, junho de 2003.

PERES, A. M. M.; REYNOLDS, A. C. Theory and Analysis od Injectivity Tests on Horizontal Wells. Society of Petroleum Engineers, SPE Journal, SPE Paper 90907, setembro de 2006.

ROSA, A.J; CARVALHO, R.S.; XAVIER, J.A.D. Engenharia de Reservatórios de Petróleo. Editora Interciência. Rio de Janeiro, 2011.

THOMPSON, L.G.; REYNOLDS, A.C. Well Testing for Radially Heterogeneous Reservoirs Under Single and Multiphase Flow Conditions. SPE Formation Evaluation, SPE Paper 30577, setembro de março de 1997.

SCILAB. Version 6.0.1. ESI Group, 2018. Disponível em: $<$ https://www.scilab.org/>. Acesso em: 21 jan. 2019.

IMEX. Version 2017.10. CMG, 2017. Disponível em: < https://www.cmgl.ca/>. Acesso em: 21 jan. 2019. 


\section{Apêndice A}

- Solução do raio da frente de avanço em coordenadas cilíndricas (Teoria de Buckley-Leverett para escoamento radial)

A injeção de água no reservatório, visa o aumento da recuperação do óleo contido no reservatório, e pode ser modelada utilizando-se a teoria de Buckley-Leverett, que permite a análise do fluxo unidimensional e bifásico em um meio poroso. Esse modelo pode ser entendido como um pistão com vazamento, onde um banco de água é injetado deslocando o óleo do reservatório, entretanto esse deslocamento não é completo, restando uma saturação de óleo na região já varrida pela água (OLIVEIRA, 2014).

No fluxo bifásico, a vazão total, $q_{t}$, é a soma das vazões de água, $q_{w}$, e óleo, $q_{o}$ :

$$
q_{t}(r, t)=q_{w}(r, t)+q_{o}(r, t)
$$

De forma que o fluxo fracionário de água, $f_{w}$, é definido como a razão entre a vazão da água e a vazão total:

$$
f_{w}=\frac{q_{w}(r, t)}{q_{t}(r, t)}
$$

Considerando que as vazões do óleo e da água são, respectivamente,

$$
q_{o}(r, t)=-2 \pi r h v_{o}
$$




$$
q_{w}(r, t)=-2 \pi r h v_{w}
$$

e que pela Lei de Darcy:

$$
v_{o}(r, t)=\frac{-k \cdot k_{r o}}{\mu_{o}} \frac{\partial p}{\partial r}
$$

e

$$
v_{w}(r, t)=\frac{-k \cdot k_{r w}}{\mu_{w}} \frac{\partial p}{\partial r}
$$

Substituindo as equações ( 144 ) e ( 145 ) em ( 142 ) e ( 143 ), respectivamente:

$$
q_{o}(r, t)=\frac{2 \pi r h k \cdot k_{r o}}{\mu_{o}} \frac{\partial p}{\partial r}
$$

$$
q_{w}(r, t)=\frac{2 \pi r h k \cdot k_{r w}}{\mu_{w}} \frac{\partial p}{\partial r}
$$

Substituindo as equações ( 146 ) e ( 147 ) em ( 141 ), podemos escrever:

$$
\begin{aligned}
f_{w} & =\frac{\left(\frac{2 \pi r h k \cdot k_{r w}}{\mu_{w}} \frac{\partial p}{\partial r}\right)}{\left(\frac{2 \pi r h k \cdot k_{r o}}{\mu_{o}} \frac{\partial p}{\partial r}\right)+\left(\frac{2 \pi r h k \cdot k_{r w}}{\mu_{w}} \frac{\partial p}{\partial r}\right)} \\
f_{w} & =\frac{\left(\frac{k_{r w}}{\mu_{w}}\right)}{\left(\frac{k_{r o}}{\mu_{o}}\right)+\left(\frac{k_{r w}}{\mu_{w}}\right)}
\end{aligned}
$$

Para $\mu_{w}$ e $\mu_{o}$ constantes, $f_{w}=f_{w}\left(S_{w}\right)$. 
As equações da continuidade das fases água e óleo são dadas, respectivamente, por:

$$
\begin{gathered}
\frac{1}{r} \frac{\partial}{\partial r}\left(r \rho_{o} v_{o}\right)=-\frac{\partial}{\partial t}\left(\phi \rho_{o} S_{o}(r, t)\right) \\
\frac{1}{r} \frac{\partial}{\partial r}\left(r \rho_{w} v_{w}\right)=-\frac{\partial}{\partial t}\left(\phi \rho_{w} S_{w}(r, t)\right)
\end{gathered}
$$

Considerando a pressão capilar $\left(p_{c}\right)=0$ e rocha e fluido incompressíveis ( $c_{r}=0$ e $\rho_{w}, \rho_{o}=$ constantes $)$ :

$$
\rho_{o} \frac{1}{r} \frac{\partial}{\partial r}\left(r v_{o}\right)=-\phi \rho_{o} \frac{\partial S_{o}(r, t)}{\partial t}
$$

$$
\rho_{w} \frac{1}{r} \frac{\partial}{\partial r}\left(r v_{w}\right)=-\phi \rho_{w} \frac{\partial S_{w}(r, t)}{\partial t}
$$

Ou ainda:

$$
\frac{1}{r} \frac{\partial}{\partial r}\left(r v_{o}\right)=-\phi \frac{\partial S_{o}(r, t)}{\partial t}
$$

$\frac{1}{r} \frac{\partial}{\partial r}\left(r v_{w}\right)=-\phi \frac{\partial S_{w}(r, t)}{\partial t}$

Multiplicando os dois lados da equação por $2 \pi h$ :

$$
\begin{aligned}
& \frac{1}{r} \frac{\partial}{\partial r}\left(2 \pi h r v_{o}\right)=-2 \pi h \phi \frac{\partial S_{o}(r, t)}{\partial t} \\
& \frac{1}{r} \frac{\partial}{\partial r}\left(2 \pi h r v_{w}\right)=-2 \pi h \phi \frac{\partial S_{w}(r, t)}{\partial t}
\end{aligned}
$$


Observa-se que o termo entre parênteses do lado esquerdo das equações se equivale as vazões no em um regime de fluxo radial das fases $q_{o}(r, t)$ e $q_{w}(r, t)$, de forma que:

$$
\begin{aligned}
& \frac{1}{r} \frac{\partial}{\partial r}\left(q_{o}(r, t)\right)=-2 \pi h \phi \frac{\partial S_{o}(r, t)}{\partial t} \\
& \frac{1}{r} \frac{\partial}{\partial r}\left(q_{w}(r, t)\right)=-2 \pi h \phi \frac{\partial S_{w}(r, t)}{\partial t}
\end{aligned}
$$

No caso da fase água, podemos escrever ainda:

$$
\frac{1}{r} \frac{\partial}{\partial r}\left(f_{w}\left(S_{w}\right) q_{t}(r, t)\right)=-2 \pi h \phi \frac{\partial S_{w}(r, t)}{\partial t}
$$

Em regime permanente e constante para todo $r$ e $t$, a vazão $q_{t}$ é igual a vazão do poço, $q B_{w}$. Assim:

$$
\begin{gathered}
q_{t} \frac{\partial}{\partial r}\left(f_{w}\left(S_{w}\right)\right)=-2 \pi h \phi r \frac{\partial S_{w}(r, t)}{\partial t} \\
\frac{\partial}{\partial r}\left(f_{w}\left(S_{w}\right)=-\frac{2 \pi h \phi r}{q B_{w}} \frac{\partial S_{w}(r, t)}{\partial t}\right.
\end{gathered}
$$

Usando a regra da cadeia no lado esquerdo:

$$
\frac{\partial f_{w}}{\partial S_{w}} \frac{\partial S_{w}(r, t)}{\partial r}=-\frac{2 \pi h \phi r}{q B_{w}} \frac{\partial S_{w}(r, t)}{\partial t}
$$

Além disso, $f_{w}{ }^{\prime}=\frac{\partial f_{w}}{\partial S_{w}}$ representa a derivada da curva de fluxo fracionário na saturação $S_{w}$. Assim,

$$
\frac{\partial S_{w}(r, t)}{\partial t}+\frac{q B_{w}}{2 \pi h \phi r} f_{w}{ }^{\prime} \frac{\partial S_{w}(r, t)}{\partial r}=0
$$


Que é uma EDP hiperbólica de 1a ordem. Como o coeficiente da derivada espacial é função da variável dependente $S_{w}$, a equação é uma EDP quase-linear.

Sendo $S_{w}=S_{w}(r, t)$, o diferencial de $S_{w}$ é dado por:

$$
d S_{w}=\left.\frac{\partial S_{w}}{\partial t}\right|_{r} d t+\left.\frac{\partial S_{w}}{\partial r}\right|_{t} d r
$$

Uma trajetória no plano $r-t$ na qual a variação de $S_{w}$ é nula $\left(d S_{w}=\right.$ 0) satisfaz a seguinte relação:

$$
\left.\frac{\partial S_{w}}{\partial t}\right|_{r} d t=-\left.\left.\frac{\partial S_{w}}{\partial r}\right|_{t} d r \Rightarrow \frac{d r}{d t}\right|_{S_{w}}=-\frac{\left.\frac{\partial S_{w}}{\partial t}\right|_{t}}{\left.\frac{\partial S_{w}}{\partial r}\right|_{r}}
$$

Portanto, substituindo na equação,

$$
\left.\frac{d r}{d t}\right|_{S_{w}}=\frac{q B_{w}}{2 \pi h \phi r} f_{w}{ }^{\prime}\left(S_{w}\right)
$$

ou

$$
\left.\frac{d\left(r^{2}\right)}{d t}\right|_{S_{w}}=\frac{q B_{w}}{\pi h \phi} f_{w}{ }^{\prime}\left(S_{w}\right)
$$

Para conhecer a trajetória de um certo valor de $S_{w}$, basta integrar a equação de $\mathrm{t}=0$ a t.

$$
\left.\int_{0}^{t} \frac{d\left(r^{2}\right)}{d t}\right|_{S_{w}} d t=\int_{0}^{t} \frac{q B_{w}}{\pi h \phi} f_{w}{ }^{\prime}\left(S_{w}\right) d t
$$




$$
r^{2}\left(S_{w}\right)-r^{2}{ }_{w}\left(S_{w}\right)=\frac{q B_{w}}{\pi h \phi} f_{w}{ }^{\prime}\left(S_{w}\right) t
$$

A equação acima é a equação de Buckley-Leverett para o problema radial, onde $r^{2}\left(S_{w}\right)$ é a posição de $S_{w}$ em te $r^{2}{ }_{w}\left(S_{w}\right)$ é a posição de $S_{w}$ em $\mathrm{t}=0$.

Destaca-se que a solução proposta se aplica essencialmente à fluidos incompressíveis com efeitos difusivos (devido à pressão capilar) desprezados. Nesse trabalho essa hipótese é assumida tendo em vista que os tempos utilizados nos testes são pequenos. 


\section{Apêndice B}

- Derivada de Bourdet

Bourdet propôs a utilização da derivada da pressão em relação ao log natural do tempo para interpretar testes de formação, de forma que:

$$
\begin{aligned}
& p^{\prime}{ }_{w D}=\frac{d p_{w D}}{d \ln t_{D}} \\
& p^{\prime}{ }_{w D}=t_{D} \frac{d p_{w D}}{d t_{D}}
\end{aligned}
$$

Onde $p^{\prime}{ }_{w D}$ representa a derivada logarítmica da pressão adimensional $p_{w D}$. Usando as definições adimensionais, é possível mostrar que:

$$
\begin{aligned}
& p_{w D}=\frac{k h \Delta p^{\prime}}{\alpha_{p} q B \mu} \\
& \Delta p^{\prime}=\frac{d \Delta p}{d \ln t} \\
& \Delta p^{\prime}=t \frac{d \Delta p}{d t}
\end{aligned}
$$


Como as pressões são medidas de forma discreta, usualmente o algoritmo mais utilizado para calcular a derivada da pressão, proposto por Bourdet, utiliza 3 pontos para calcular uma derivada no ponto " $X$ ", conforme indicado na figura abaixo.

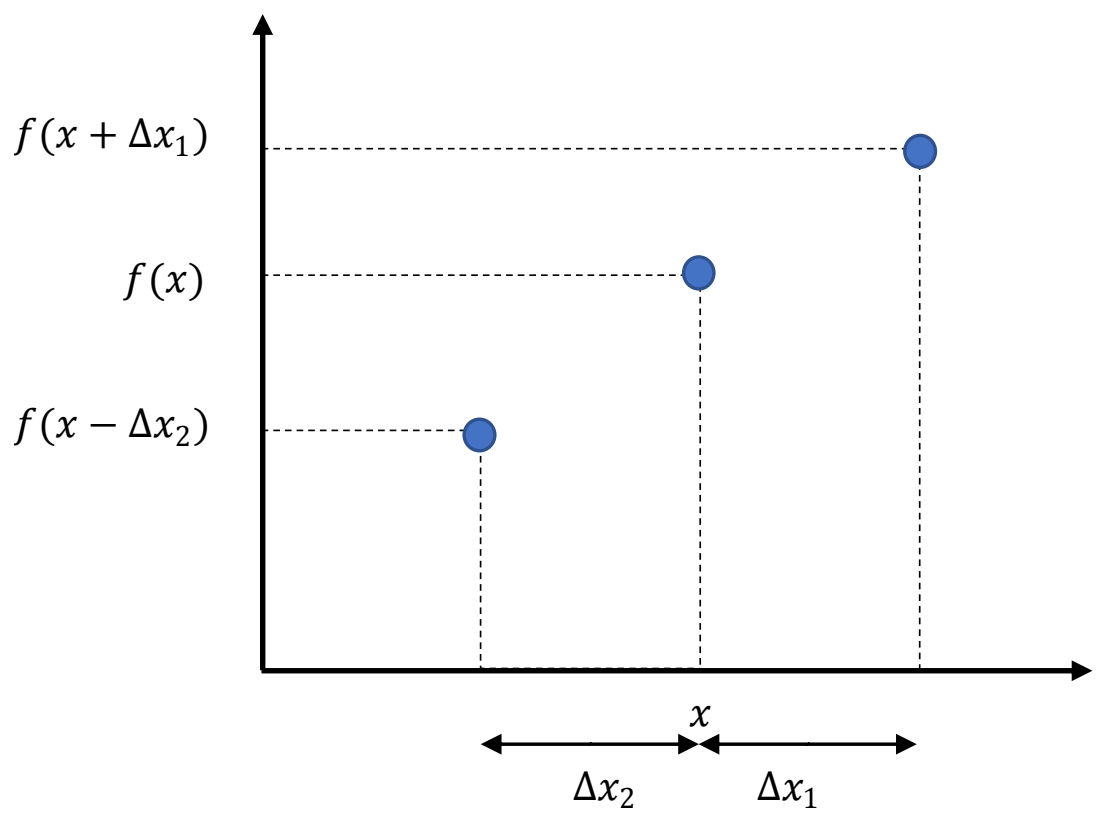

Figura 38 - Representação de uma função f(x)

Expandindo a função "f" em torno de "x" por meio da série de Taylor, pode-se escrever:

$$
\begin{aligned}
f^{\prime}(x)=\frac{f\left(x+\Delta x_{1}\right)-f(x)}{\Delta x_{1}} \frac{\Delta x_{2}}{\Delta x_{1}+\Delta x_{2}} \\
+\frac{f\left(x+\Delta x_{2}\right)-f(x)}{\Delta x_{2}} \frac{\Delta x_{1}}{\Delta x_{1}+\Delta x_{2}}
\end{aligned}
$$

No caso de testes de formação, em que se deseja escrever a derivada da pressão em relação ao log natural, a representação é dada na figura abaixo 


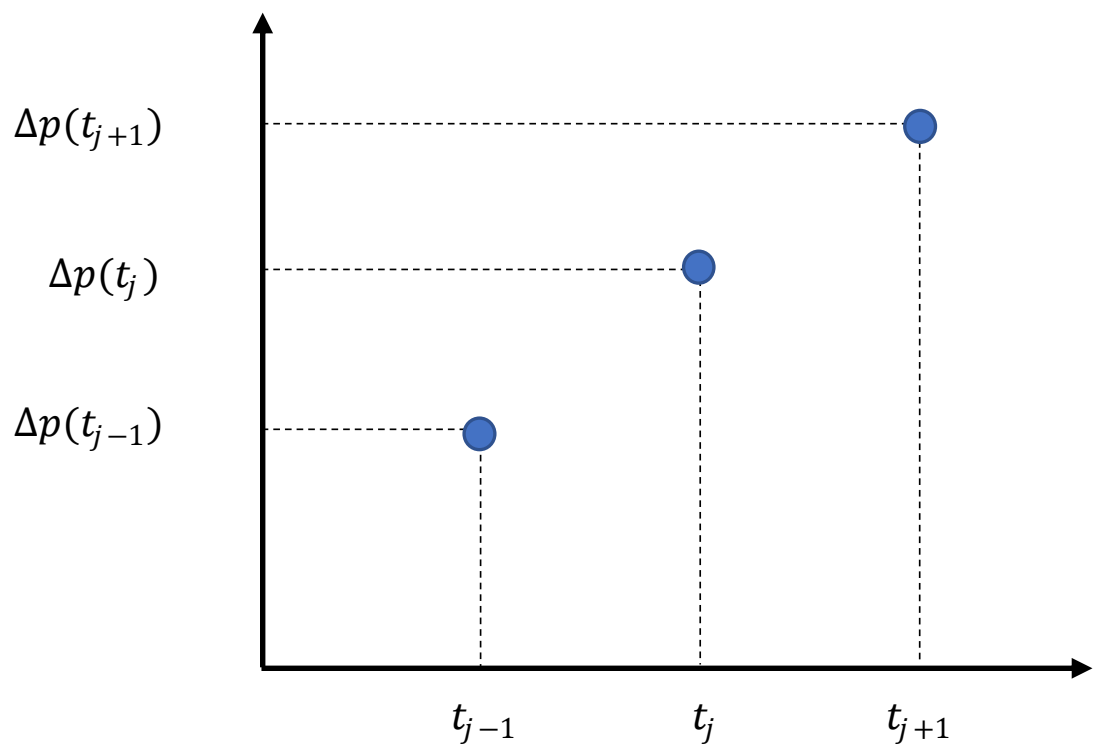

Figura 39 - Representação dos pontos de medição de pressão

E pode-se, analogamente, escrever:

$$
\begin{aligned}
\Delta p^{\prime}\left(t_{j}\right)=\frac{\Delta p\left(t_{j+1}\right)-\Delta p\left(t_{j}\right)}{\ln \left(\frac{t_{j+1}}{t_{j}}\right)} \frac{\ln \left(\frac{t_{j}}{t_{j-1}}\right)}{\ln \left(\frac{t_{j+1}}{t_{j-1}}\right)} \\
+\frac{\Delta p\left(t_{j}\right)-\Delta p\left(t_{j-1}\right)}{\ln \left(\frac{t_{j}}{t_{j-1}}\right)} \frac{\ln \left(\frac{t_{j+1}}{t_{j}}\right)}{\ln \left(\frac{t_{j+1}}{t_{j-1}}\right)}
\end{aligned}
$$

Para os primeiro e último pontos, como não é possível utilizar o algoritmo de Bourdet, que exige três pontos, pode-se calcular a derivada da forma tradicional. 


\section{Apêndice C}

- Variáveis Admensionais

Buscando simplificar os cálculos e equações, nesse trabalho, em algumas ocasiões, optou-se por trabalhar com grandezas adimensionais, ou seja, sem unidade física que os defina.

Esse apêndice apresenta o desenvolvimento dessas variáveis, que são representadas pelo subscrito $D$.

Para adimensionalizar o raio no reservatório, essa grandeza é dividida pelo raio do poço, $r_{w}$.

$$
r_{D}=\frac{r}{r_{w}}
$$

Já para adimensionalizar o tempo e o tempo de produção buscou-se a simplificação da solução monofásica:

$$
p\left(r_{w}, t\right)-p_{i}=\frac{\tilde{q}}{2 \pi k \hat{\lambda}_{o} h}\left[-\frac{1}{2} E_{i}\left(-\frac{r^{2} \phi \hat{c}_{t o}}{4 k \hat{\lambda}_{o} t}\right)\right]
$$

Dessa forma, se optou pela adimensionalização por meio das razões dadas pelas equações abaixo.

$$
\begin{aligned}
& t_{D}=\frac{\hat{\lambda}_{o} k t}{c_{t o} \phi r_{w}{ }^{2}} \\
& t_{p D}=\frac{\hat{\lambda}_{o} k t_{p}}{c_{t o} \phi r_{w}{ }^{2}}
\end{aligned}
$$

A queda de pressão adimensional para o fluxo radial foi adotada como: 
$p_{D}=\frac{2 \pi k h \hat{\lambda}_{o}}{q} \Delta p$

Sendo assim a equação ( 179 ) pode ser escrita como:

$p_{D}\left(r_{D}, t_{D}\right)=-\frac{1}{2} E_{i}\left(-\frac{r_{D}^{2}}{4 t_{D}}\right)$

A vazão $q_{D}$ é dada por:

$q_{D}=\frac{q}{q_{\text {inj }}}$ 


\section{Apêndice D}

\section{- Algoritmo Desenvolvido no software Scilab}

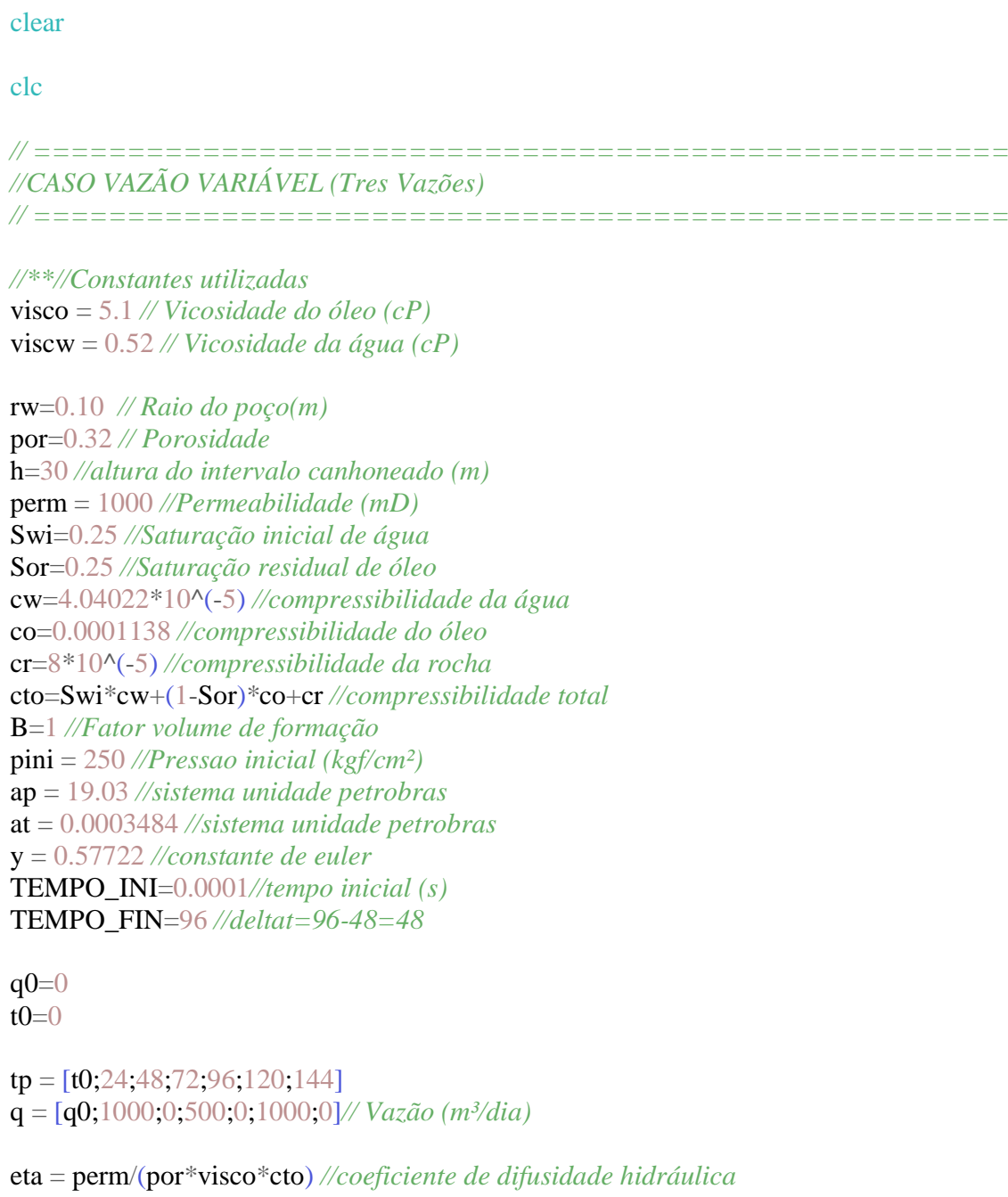

$\mathrm{q} 0=0$

$\mathrm{t} 0=0$

tp $=[\mathrm{t} 0 ; 24 ; 48 ; 72 ; 96 ; 120 ; 144]$

$\mathrm{q}=[\mathrm{q} 0 ; 1000 ; 0 ; 500 ; 0 ; 1000 ; 0] / / \operatorname{Vaz} \tilde{a} o\left(\mathrm{~m}^{3} /\right.$ dia $)$

eta $=$ perm/(por*visco*cto) //coeficiente de difusidade hidráulica

NPONTOS_KREL=27//Numero de pontos da curva de permeabilidade relativas, cujos valores para água e óleo são dados abaixo:

$\operatorname{Sw}(01)=0.2500$; $\operatorname{Sw}(02)=0.2525$ $\operatorname{Sw}(03)=0.2550$; $\mathrm{Sw}(04)=0.2600$ $\operatorname{Sw}(05)=0.2650$; $\operatorname{Sw}(06)=0.2700$ $\mathrm{Sw}(07)=0.2750$ $\operatorname{Sw}(08)=0.2875$ $\mathrm{Sw}(09)=0.3000$ $\operatorname{Sw}(10)=0.3250$; $\mathrm{Sw}(11)=0.3500$ $\operatorname{Sw}(12)=0.4000$;

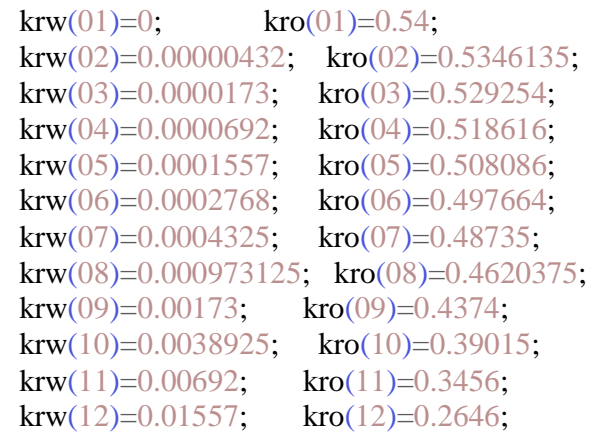



$\operatorname{Sw}(13)=0.4500$;
$\mathrm{Sw}(14)=0.5000$;
$\mathrm{Sw}(15)=0.5500$
$\mathrm{Sw}(16)=0.6000$;
$\mathrm{Sw}(17)=0.6500$;
$\mathrm{Sw}(18)=0.6750$
$\operatorname{Sw}(19)=0.7000$
$\operatorname{Sw}(20)=0.7125$
$\operatorname{Sw}(21)=0.7250$;
$\operatorname{Sw}(22)=0.7300$
$\operatorname{Sw}(23)=0.7350$;
$\operatorname{Sw}(24)=0.7400$;
$\mathrm{Sw}(25)=0.7450$
$\operatorname{Sw}(26)=0.7475$
$\operatorname{Sw}(27)=0.7500$;



//**//Mostra a curva das permeabilidades relativas em função da saturação de água.

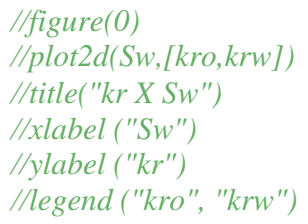

M_chapeu=mobWchapeu/mobOchapeu

//figure(1)

$/ / p l o t(S w,[f w])$

//title("fw X Sw")

Ixlabel ("Sw")

//ylabel ("fw")

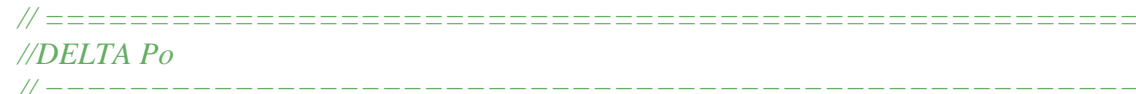

$\mathrm{i}=1$

deltat=TEMPO_INI

$\mathrm{t}(\mathrm{i})=\mathrm{tp}(1)+$ TEMPO_INI

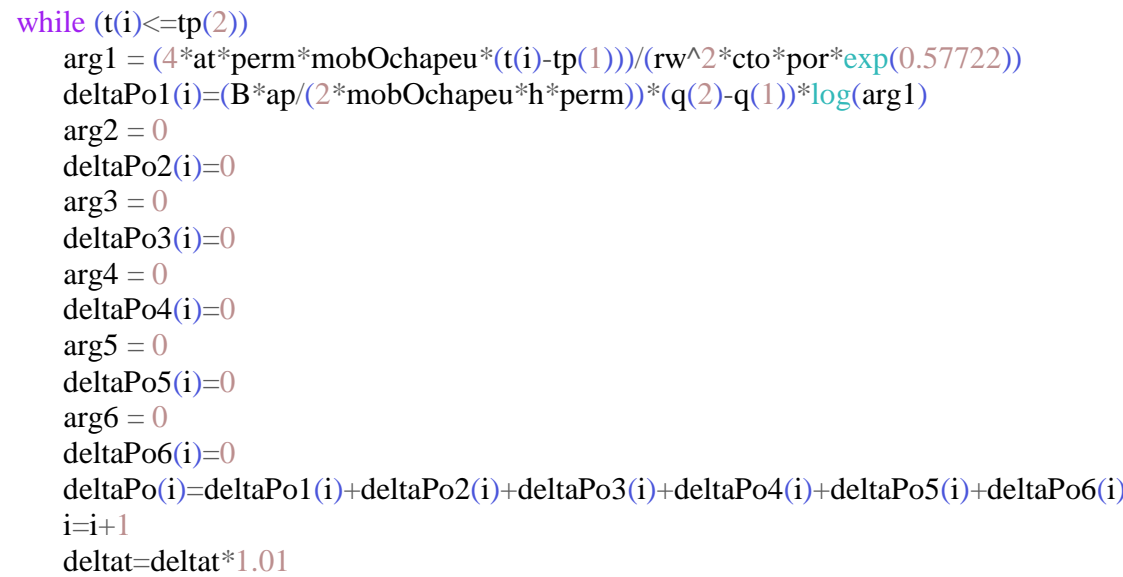









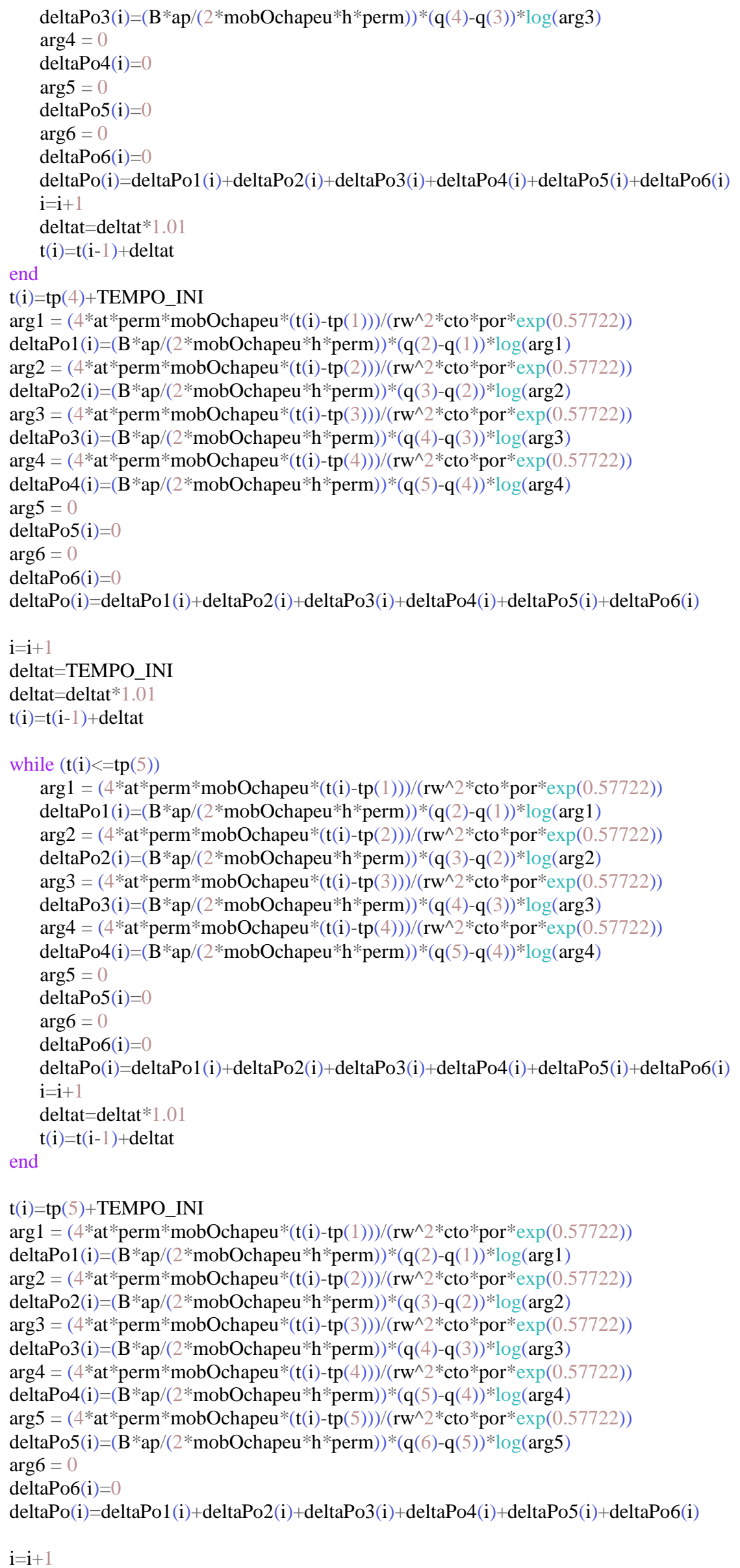







deltaPo3(i) $=(\mathbf{B} *$ ap $/(2 *$ mobOchapeu*h*perm $) *(\mathbf{q}(4)-\mathbf{q}(3)) * \log (\arg 3)$

$\arg 4=(4 *$ at $*$ perm $*$ mobOchapeu $*(\mathbf{t}(\mathbf{i})-\operatorname{tp}(4))) /\left(\mathbf{r w}^{\wedge} 2 *\right.$ cto $^{*}$ por*exp $\left.(0.57722)\right)$

deltaPo4$(\mathbf{i})=(\mathbf{B} * \mathrm{ap} /(2 *$ mobOchapeu*h*perm $)) *(\mathbf{q}(5)-\mathbf{q}(4)) * \log (\arg 4)$

$\arg 5=\left(4 * a t^{*}\right.$ perm $*$ mobOchapeu* $\left.*(\mathbf{t}(\mathbf{i})-\operatorname{tp}(5))\right) /\left(\mathbf{r w}^{\wedge} 2 *\right.$ cto*por*exp $\left.(0.57722)\right)$

$\operatorname{deltaPo5}(\mathbf{i})=(\mathbf{B} * \operatorname{ap} /(2 *$ mobOchapeu*h*perm $)) *(\mathbf{q}(6)-\mathrm{q}(5)) * \log (\arg 5)$

$\arg 6=\left(4 * a t^{*}\right.$ perm $*$ mobOchapeu* $\left.(\mathbf{t}(\mathbf{i})-\operatorname{tp}(6))\right) /\left(\mathbf{r w}^{\wedge} 2 *\right.$ cto $^{*}$ por*exp $\left.(0.57722)\right)$

deltaPo6(i) $=(\mathbf{B} *$ ap $/(2 *$ mobOchapeu*h*perm $) *(\mathbf{q}(7)-\mathrm{q}(6)) * \log (\arg 6)$

deltaPo $(\mathrm{i})=\operatorname{deltaPo} 1(\mathrm{i})+\operatorname{deltaPo} 2(\mathrm{i})+\operatorname{deltaPo} 3(\mathrm{i})+\operatorname{deltaPo} 4(\mathrm{i})+\operatorname{deltaPo} 5(\mathrm{i})+\operatorname{deltaPo6}(\mathrm{i})$

figure(6)

plot2d (t, [deltaPo],logflag='nn')

title ("deltaPo x t")

ylabel ("deltaPo")

xlabel ("t")

//Derivada de Bourdet do Fluxo Fracionário

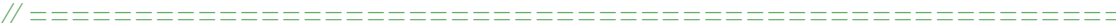

for $\mathbf{n}=2:($ NPONTOS_KREL-1)

$\operatorname{derfw}(\mathbf{n})=((\mathbf{f w}(\mathrm{n}+1)-\mathrm{fw}(\mathrm{n})) /(\operatorname{Sw}(\mathbf{n}+1)-\operatorname{Sw}(\mathbf{n}))) *(\operatorname{Sw}(\mathbf{n})-\operatorname{Sw}(\mathbf{n}-1)) /(\operatorname{Sw}(\mathbf{n}+1)-\operatorname{Sw}(\mathbf{n}-1))+((\mathbf{f w}(\mathbf{n})-$ $\mathbf{f w}(\mathbf{n}-1)) /(\operatorname{Sw}(\mathbf{n})-\mathbf{S w}(\mathbf{n}-1))) *(\mathbf{S w}(\mathbf{n}+1)-\operatorname{Sw}(\mathbf{n})) /(\mathbf{S w}(\mathbf{n}+1)-\operatorname{Sw}(\mathbf{n}-1))$

end

//Derivada para primeiro e último termos

$\operatorname{derfw}(1)=(\mathrm{fw}(2)-\mathrm{fw}(1)) /(\operatorname{Sw}(2)-\mathrm{Sw}(1))$

$\operatorname{derfw}($ NPONTOS_KREL $)=0.0$

$\operatorname{derf} w 1=\operatorname{derf} w$

//figure(7)

//plot $(S w, \operatorname{derfw})$

//title("derfw X Sw")

//ylabel ("derfw")

//xlabel ("Sw")

for $\mathrm{n}=2$ :(NPONTOS_KREL-1)

$\operatorname{der} 2 \mathrm{fw}(\mathbf{n})=((\operatorname{derfw}(\mathbf{n}+1)-\operatorname{derfw}(\mathbf{n})) /(\operatorname{Sw}(\mathbf{n}+1)-\operatorname{Sw}(\mathbf{n}))) *(\operatorname{Sw}(\mathbf{n})-\operatorname{Sw}(\mathbf{n}-1)) /(\operatorname{Sw}(\mathbf{n}+1)-\operatorname{Sw}(\mathbf{n}-1))+($ $(\operatorname{derfw}(\mathbf{n})-\operatorname{derfw}(\mathbf{n}-1)) /(\operatorname{Sw}(\mathbf{n})-\mathbf{S w}(\mathbf{n}-1))) *(\operatorname{Sw}(\mathbf{n}+1)-\operatorname{Sw}(\mathbf{n})) /(\operatorname{Sw}(\mathbf{n}+1)-\operatorname{Sw}(\mathbf{n}-1))$

end

//Derivada simples para primeiro ponto

$\operatorname{der} 2 \mathrm{fw}(1)=(\operatorname{derfw}(2)-\operatorname{derfw}(1)) /(\operatorname{Sw}(2)-\operatorname{Sw}(1))$

der2fw(NPONTOS_KREL $)=0.0$

derfwelge $=$ derfw

derfwelge_MAX=-9999.9

for $\mathbf{n}=$ NPONTOS_KREL:-1:1

if derfwelge_MAX $<\operatorname{derfw}(\mathrm{n})$ then

derfwelge_MAX $=\operatorname{derfw}(\mathrm{n})$

$\mathrm{Sw}$ INF $=\mathrm{Sw}(\mathrm{n})$

$\mathrm{fw} \_\mathrm{INF}=\mathrm{fw}(\mathrm{n})$

end

end

der2_MAX $=1000$

$\mathrm{i}=$ NPONTOS_KREL

while $(\operatorname{der} 2 \mathrm{fw}(\mathrm{i})<$ der2_MAX)

Sw_INF=Sw(i)

fw_INF $=f w(i)$

derfw_INF $=\operatorname{derfw}(\mathrm{i})$

der2_MAX $=\operatorname{der} 2 \mathrm{fw}(\mathrm{i})$

$\mathrm{i}=\mathrm{i}-1$ 


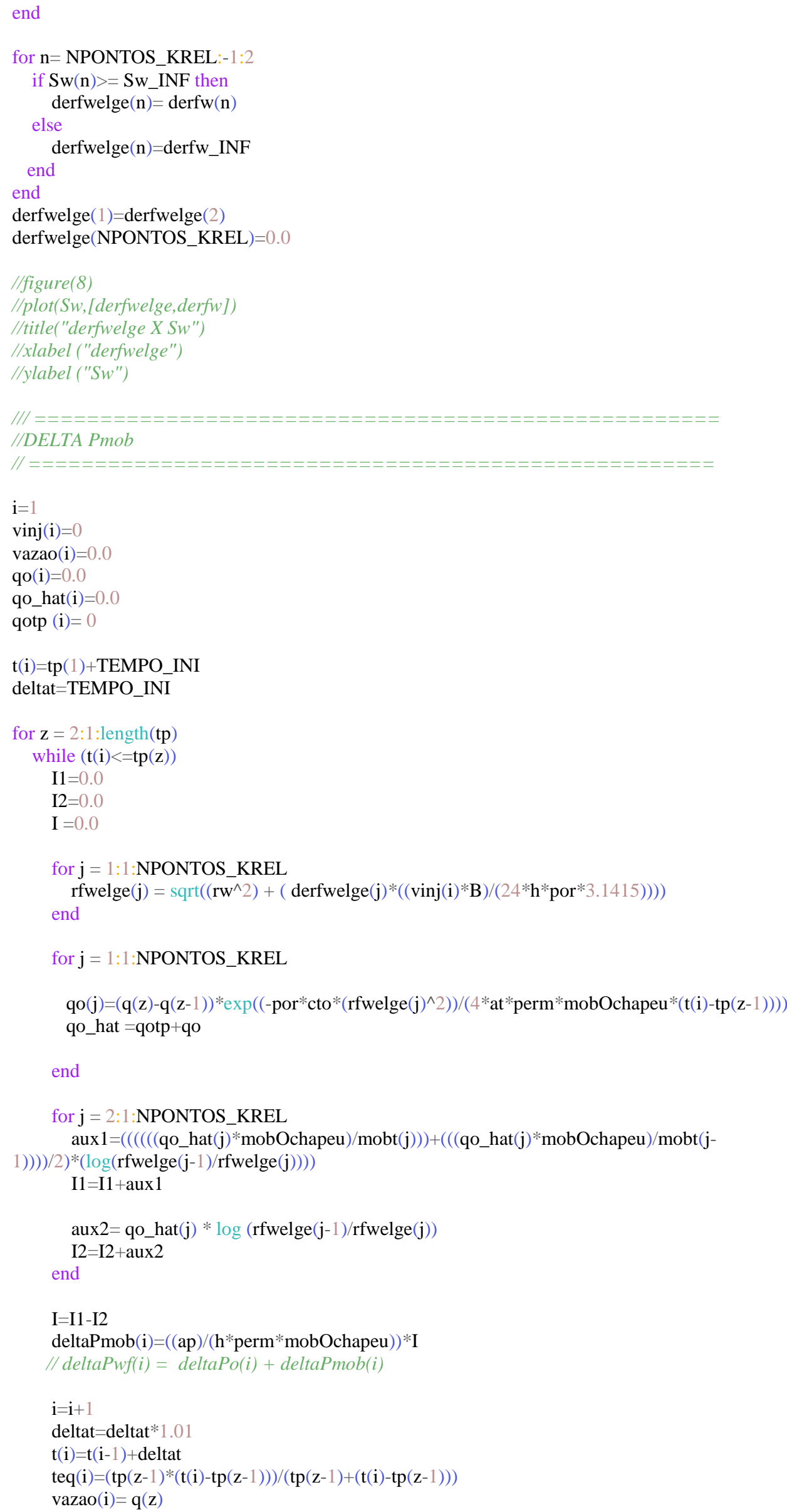




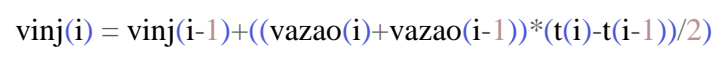


title ("Pwf x t")

ylabel ("Pwf")

xlabel ("t")

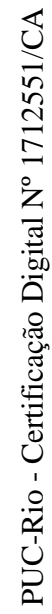

